\title{
SuperWASP observations of pulsating Am stars ${ }^{\star}$
}

\author{
B. Smalley ${ }^{1}$, D. W. Kurtz ${ }^{2}$, A. M. S. Smith ${ }^{1}$, L. Fossati ${ }^{3}$, D. R. Anderson ${ }^{1}$, S. C. C. Barros ${ }^{4}$, O. W. Butters ${ }^{5}$, \\ A. Collier Cameron ${ }^{6}$, D. J. Christian ${ }^{7}$, B. Enoch ${ }^{6}$, F. Faedi ${ }^{4}$, C. A. Haswell ${ }^{3}$, C. Hellier ${ }^{1}$, S. Holmes ${ }^{3}$, K. Horne ${ }^{6}$, \\ S. R. Kane ${ }^{8}$, T. A. Lister ${ }^{9}$, P. F. L. Maxted ${ }^{1}$, A. J. Norton $^{3}$, N. Parley ${ }^{6}$, D. Pollacco ${ }^{4}$, E. K. Simpson ${ }^{4}$, I. Skillen ${ }^{10}$, \\ J. Southworth ${ }^{1}$, R. A. Street ${ }^{9}$, R. G. West ${ }^{5}$, P. J. Wheatley ${ }^{11}$, and P. L. Wood ${ }^{1}$
}

\author{
1 Astrophysics Group, Keele University, Staffordshire, ST5 5BG, UK \\ e-mail: bs@astro.keele.ac.uk \\ 2 Jeremiah Horrocks Institute of Astrophysics, University of Central Lancashire, Preston PR1 2HE, UK \\ 3 Department of Physics \& Astronomy, The Open University, Walton Hall, Milton Keynes, MK7 6AA, UK \\ 4 Astrophysics Research Centre, Main Physics Building, School of Mathematics \& Physics, Queen's University, University Road, \\ Belfast, BT7 1NN, UK \\ 5 Department of Physics \& Astronomy, University of Leicester, Leicester, LE1 7RH, UK \\ ${ }^{6}$ SUPA, School of Physics \& Astronomy, University of St. Andrews, North Haugh, Fife, KY16 9SS, UK \\ 7 Department of Physics \& Astronomy, California State University, Northridge, CA, 91330, USA \\ 8 NASA Exoplanet Science Institute, Caltech, MS 100-22, 770 South Wilson Avenue, Pasadena, CA, 91125, USA \\ ${ }^{9}$ Las Cumbres Observatory Global Telescope Network, 6740 Cortona Drive, Suite 102, Goleta, CA, 93117, USA \\ ${ }^{10}$ Isaac Newton Group of Telescopes, Apartado de Correos 321, 38700 Santa Cruz de la Palma, Tenerife, Spain \\ 11 Department of Physics, University of Warwick, Coventry, CV4 7AL, UK
}

Received 10 May 2011 / Accepted 30 June 2011

\section{ABSTRACT}

\begin{abstract}
We have studied over 1600 Am stars at a photometric precision of 1 mmag with SuperWASP photometric data. Contrary to previous belief, we find that around 200 Am stars are pulsating $\delta$ Sct and $\gamma$ Dor stars, with low amplitudes that have been missed in previous, less extensive studies. While the amplitudes are generally low, the presence of pulsation in Am stars places a strong constraint on atmospheric convection, and may require the pulsation to be laminar. While some pulsating Am stars have been previously found to be $\delta$ Sct stars, the vast majority of Am stars known to pulsate are presented in this paper. They will form the basis of future statistical studies of pulsation in the presence of atomic diffusion.
\end{abstract}

Key words. asteroseismology - stars: chemically peculiar - stars: oscillations - stars: variables: delta Scuti - techniques: photometric

\section{Introduction}

In the region of the Hertzsprung-Russell (HR) diagram where the Cepheid instability strip extends across the main sequence, there is a complex relationship between stellar pulsation and atmospheric abundance anomalies that is not fully understood. This region ranges from the early A stars to mid-F stars in spectral type, and from the zero age main sequence to the terminal age main sequence in luminosity. Found here are the strongly magnetic chemically peculiar Ap and Fp stars, the non-magnetic metallic-lined Am stars, the rarer metal-deficient $\lambda$ Boo stars, the pulsating $\delta$ Sct stars, $\gamma$ Dor stars and rapidly oscillating Ap (roAp) stars. Much has been written about these stars and their physics, which we briefly summarise here. For more detailed discussions see Joshi et al. (2006), Kurtz \& Martinez (2000) and Kurtz (1989, 1978, 1976).

Most stars in the main-sequence region of the instability strip are normal abundance $\delta$ Sct stars with relatively high rotational velocities - usually $v \sin i \geq 100 \mathrm{~km} \mathrm{~s}^{-1}$. A large fraction of A stars are Am stars, peaking at around 50 per cent at A8, but Am stars are believed either not to pulsate as $\delta$ Sct stars, or may do so with much smaller amplitudes than the normal abundance $\delta$ Sct stars. Am stars are mostly found in short period binary

* An extended version of Table 1 containing all the detected frequencies and amplitudes is only available at the CDS via anonymous ftp to cdsarc.u-strasbg. fr (130.79.128.5) or via http://cdsarc.u-strasbg.fr/viz-bin/qcat?]/A+A/535/A3 systems with orbital periods between 1-10 d, causing synchronous rotation with $v \sin i \leq 120 \mathrm{~km} \mathrm{~s}^{-1}$ (Abt 2009); a few single Am stars with similar slow rotation are known.

The magnetic Ap stars are rarer, constituting less than 10 per cent of the A stars. They have very strong global magnetic fields and are often roAp stars with high overtone p mode pulsations with much shorter periods than the $\delta$ Sct stars. No Ap star is known to be a $\delta$ Sct star. Our physical understanding is that atomic diffusion - radiative levitation and gravitational settling - stabilises the slowly rotating Am and Ap stars so that low overtone p modes are not excited; particularly important in this context is the gravitational settling of helium from the He II ionisation zone where the $\kappa$-mechanism drives the pulsation of $\delta$ Sct stars (see Aerts et al. 2010). Otherwise, the more rapidly rotating stars remain mixed because of turbulence induced by meridional circulation and are excited by the $\kappa$-mechanism (Turcotte et al. 2000).

The understanding of the relationship of the long-established $\delta$ Sct stars to the more recently discovered $\gamma$ Dor stars is currently in flux. Previously, the $\delta$ Sct stars were known as p mode pulsators, while the $\gamma$ Dor stars were known as g mode pulsators. The instability strips for these classes of stars partially overlap, and some "hybrid" stars were discovered with pulsation in both p modes and g modes. A striking case is that of HD 8801, which is an Am star that shows both $\delta$ Sct and $\gamma$ Dor p-mode and g-mode pulsation (Henry \& Fekel 2005). 
Hybrid stars that show both p modes and g modes are of particular interest asteroseismically because the p modes characterise the conditions primarily in the outer part of the star, while the g modes test the core conditions. Now with data from the Kepler Mission, which is obtaining nearly continuous data for over 150000 stars for $3.5 \mathrm{y}$, mostly with 30-min cadence, but for 512 stars with 1-min cadence (Gilliland et al. 2010), the Kepler Asteroseismic Science Consortium (KASC) is studying numbers of $\delta$ Sct stars and $\gamma$ Dor stars at $\mu$ mag precision. It is becoming clear that hybrid stars are common and may be the norm, so that the classes of $\delta$ Sct and $\gamma$ Dor stars are merging (Grigahcène et al. 2010). Interestingly, the latter authors find a possible correlation among the hybrid stars and Am spectral classification.

The Kepler Mission through KASC will model individual Am stars that are $\delta$ Sct pulsators with data of such high precision that new insight into the physics of the relationship between atomic diffusion and p mode pulsation will be obtained. But Kepler has a limited number of Am stars in its $105 \mathrm{deg}^{2}$ field-of-view. Another complementary source of information is to look at the statistics of pulsation in Am stars over the entire sky. That is now possible with the highly successful SuperWASP planetary transit-finding programme (Pollacco et al. 2006) that has surveyed a large fraction of both the northern and southern skies. There now exists in the SuperWASP archive over 290 billion photometric measurements for more than 30 million stars. These light curves encompass many types of stars, including the A stars in general, and Am stars in particular.

In this paper we have selected Am stars from the Renson \& Manfroid (2009) catalogue of peculiar stars for which we have at least 1000 data points in SuperWASP light curves. While we do not detect pulsation in all of our programme stars, for around 200 metallic-lined stars out of over 1600 tested we find $\delta$ Sct pulsation. This is contrary to previous understanding that Am stars are constant in brightness. The reason we have gained this new understanding is that there has been no previous survey of so many Am stars, and previous studies have not all reached the SuperWASP detection threshold of only $1 \mathrm{mmag}$.

Many Am stars therefore do pulsate, generally with lower amplitude than normal abundance $\delta$ Sct stars. This amplitude difference is still to be understood in terms of atomic diffusion reducing pulsation driving for the slowly rotating Am stars, but there is not a complete lack of pulsation. That, has implications for turbulence in the diffusive layers and may require that the pulsation be laminar. Some striking examples of metallic-lined stars with relatively high pulsation amplitude (these are rare) address this question further, such as HD 188136 (Kurtz 1980; Wegner 1981) and HD 40765 (Kurtz et al. 1995). More constraints on the physics of the interaction of pulsation and atomic diffusion may also be found in stars that show no $\delta$ Sct p modes or $\gamma$ Dor g modes at precisions of $\mu$ mag. Some such A stars are known in the CoRoT and Kepler data sets, but in-depth studies have not yet been made, hence discussions of these have yet to be published.

The combination of the all-sky mmag precision of SuperWASP with the $\mu$ mag precision of CoRoT and Kepler on selected stars, calls for new attempts to model the physics of the interaction of pulsation, rotation and atomic diffusion in the A stars.

\section{Observations}

The WASP project is surveying the sky for transiting extrasolar planets (Pollacco et al. 2006) using two robotic telescopes, one at the Observatorio del Roque de los Muchachos on the island of La Palma in the Canary Islands, and the other at the Sutherland
Station, South African Astronomical Observatory (SAAO). Both telescopes consist of an array of eight 200-mm, f/1.8 Canon telephoto lenses and Andor CCDs, giving a field of view of $7.8^{\circ} \times 7.8^{\circ}$ and pixel size of around $14^{\prime \prime}$. The observing strategy is such that each field is observed with a typical cadence of the order of $10 \mathrm{~min}$. WASP provides good quality photometry with a precision exceeding 1 per cent per observation in the approximate magnitude range $9 \leq V \leq 12$.

The SuperWASP data reduction pipeline is described in detail in Pollacco et al. (2006). The aperture-extracted photometry from each camera on each night are corrected for primary and secondary extinction, instrumental colour response and system zero-point relative to a network of local secondary standards. The resultant pseudo- $V$ magnitudes are comparable to Tycho $V$ magnitudes. Additional systematic errors affecting all the stars are identified and removed using the SysRem algorithm of Tamuz et al. (2005). The final light curves are stored in the WASP project's searchable archive (Butters et al. 2010).

\section{Am star selection and analysis}

We have selected Am stars from the Renson \& Manfroid (2009) catalogue of peculiar stars for which we have data in the WASP archive and when individual light curves have at least 1000 data points (i.e. for a single camera and during a single season). Any stars known, or found, to be eclipsing binary systems were excluded from the analysis. Stars were also rejected when two approximately equal brightness stars were within the 3.5-pixel $\left(\sim 50^{\prime \prime}\right)$ SuperWASP photometry aperture. However, unresolved close pairs in DSS images (separation $\lesssim 2^{\prime \prime}$ ) and systems with fainter companions ( $\gtrsim 2 \mathrm{mag}$ ) were retained.

For each individual light curve, periodograms were calculated using the fast computation of the Lomb periodogram method of Press \& Rybicki (1989) as implemented in the Numerical Recipes FASPER routine (Press et al. 1992). Spectral window functions were also calculated, in order to identify peaks which had arisen due to the gaps in the observations. The periodograms were examined for any evidence of variability. Stars were rejected if the false alarm probability of the strongest peaks exceeded 0.1 (Horne \& Baliunas 1986). The remaining stars were examined in more detail using the PERIOd04 program (Lenz $\&$ Breger 2005). For stars in which variability was confirmed, frequencies continued to be selected so long as their amplitude was $>4$ times the average background of the pre-whitened residuals (Breger et al. 1993). Formal uncertainties on frequencies and amplitudes were obtained from the least-squares fitting using the method of Montgomery \& O'Donoghue (1999).

Of the 1620 Am stars initially selected, a total of 227 (14\% of the total) have been found to pulsate. The remaining 1393 stars were deemed as "not found to pulsate", since low-level pulsation could be present below the SuperWASP detection limits. Table 1 provides a summary of the pulsating Am stars. The individual periodograms and phase-folded lightcurves are presented in Fig. 1.

\section{Stellar parameters}

To place stars on the HR diagram we require values of $T_{\text {eff }}$ and $\log L$. For stars with $u v b y \beta$ photometry in the Hauck \& Mermilliod (1998) catalogue, we used the UvBYBETA code of Moon (1985) to obtain de-reddened indices, and the $\left(b-y, c_{0}\right)$ grids of Smalley \& Kupka (1997) to determine $T_{\text {eff }}$ and $\log g$. For stars with only uvby photometry the above procedure was used but without the de-reddening step. For stars without uvby photometry, Geveva photometry from Rufener (1988) was used 
Table 1. Pulsating Am stars.

\begin{tabular}{lcccccccccc}
\hline \hline Ren ID & Name & Sp. Type & $\begin{array}{c}\log T_{\text {eff }} \\
(\mathrm{K})\end{array}$ & $\begin{array}{c}\log L \\
\left(L_{\odot}\right)\end{array}$ & Method & $\begin{array}{c}\text { Freq. } \\
\left(\mathrm{d}^{-1}\right)\end{array}$ & $\begin{array}{c}\text { Amp. } \\
(\mathrm{mmag})\end{array}$ & nFreq. & $\begin{array}{c}\Delta T \\
(\mathrm{~d})\end{array}$ & Class \\
\hline 10 & HD 154A & A9mF2 & 3.862 & 0.89 & $\mathrm{a}$ & 4.7672 & 22.4 & 2 & 1135 & $\gamma$ Dor \\
110 & HD 719 & A3mF0 & 3.855 & 1.01 & $\mathrm{a}$ & 14.1368 & 3.2 & 10 & 473 & $\delta$ Sct \\
113 & HD 728 & Am $\delta$ Del & & & & 14.9268 & 2.8 & 2 & 119 & $\delta$ Sct \\
140 & HD 923 & A6mF2 & 3.908 & 1.39 & a & 18.6684 & 2.2 & 1 & 50 & $\delta$ Sct \\
210 & HD 1097 & A4mF4 Sr & 3.840 & 0.11 & a & 15.5491 & 2.0 & 2 & 50 & $\delta$ Sct \\
\hline
\end{tabular}

Notes. The full version of this table can be found at the CDS.

Table 2. Am stars with both SuperWASP and Kepler data.

\begin{tabular}{lccc}
\hline \hline KIC & Ren ID & $\begin{array}{c}\text { Max Amp } \\
(\mathrm{mmag})\end{array}$ & Ref \\
\hline 9204718 & 49340 & 0.13 & Bal \\
11445913 & 49650 & 2.5 & Cat, Bal \\
9272082 & 49840 & $<0.01$ & Bal \\
12253106 & 50070 & $<0.01$ & \\
9764965 & 50230 & 1.0 & \\
8881697 & 50420 & 1.9 & \\
11402951 & 50670 & 1.2 & Cat,Bal \\
9349245 & 51233 & $<0.1$ & \\
8703413 & 51640 & $<0.1$ & Bal \\
8323104 & 52260 & $<0.1$ & Bal \\
\hline
\end{tabular}

Notes. The second column gives the identification number (Ren ID) from the Renson \& Manfroid (2009) catalogue. Column 3 gives the amplitude (Max Amp) of the highest peak in the Kepler periodogram. Column 4 gives reference to published Kepler data: Cat: Catanzaro et al. (2011), Bal: Balona et al. (2011).

and the calibration of Künzli et al. (1997) used to determine $T_{\text {eff }}$ and $\log g$, assuming zero reddening. In all of the above cases, the Torres et al. (2010) relations were used to determine $\log L$. For stars without suitable intermediate-band photometry, but with Hipparcos parallaxes (van Leeuwen 2007), spectral energy distributions (SEDs) were constructed using literature broad-band photometry. Values of $T_{\text {eff }}$ were determined by fitting Kurucz flux distributions to the SEDs and $\log L$ determined from the bolometric flux at the earth $\left(f_{\oplus}\right)$ and the Hipparcos parallax. The typical uncertainties are estimated to be $\pm 200 \mathrm{~K}$ in $T_{\text {eff }}( \pm 0.01$ in $\left.\log T_{\text {eff }}\right)$ and \pm 0.25 in $\log L$. The stellar parameters are given in Table 1. In total around a third of the Am stars investigated have stellar parameters determined.

\section{Am stars in Kepler field}

The sky coverage of the SuperWASP survey overlaps with a large fraction of the Kepler field. For Am stars with light curves in both the Kepler Public archive and the SuperWASP database we have compared the frequencies and amplitudes. This allows us to evaluate the detection limits of SuperWASP. Of the 10 stars with both Kepler and SuperWASP data, four have clear pulsations with amplitudes $\gtrsim 1$ mmag (Table 2), while the other six stars have amplitudes below the SuperWASP detectability limit.

The PERIOD04 analysis (Table 3) shows good agreement above the nominal SuperWASP $1 \mathrm{mmag}$ amplitude limit. There is a suggestion that the amplitudes found using SuperWASP lightcurves are slightly higher than those from Kepler. In addition, the SuperWASP frequency can differ from the "true" frequency by a small integer number of $1 \mathrm{~d}^{-1}$ aliases. The comparison also shows that it is possible with SuperWASP data to detect frequencies slightly below the 1 mmag level (Fig. 2). Naturally, the variable data quality of ground-based photometry means that not all stars with suitable variability will be detected.
Table 3. Comparison between frequencies and amplitudes found in the Kepler and SuperWASP data for the four Am stars common to both.

\begin{tabular}{|c|c|c|c|c|}
\hline & \multicolumn{2}{|l|}{ Kepler } & \multicolumn{2}{|c|}{ SuperWASP } \\
\hline & $\begin{array}{l}\text { Freq. } \\
\left(d^{-1}\right)\end{array}$ & $\begin{array}{l}\text { Amp. }^{a} \\
\text { (mmag) }\end{array}$ & $\begin{array}{l}\text { Freq. } \\
\left(d^{-1}\right)\end{array}$ & $\begin{array}{c}\text { Amp. } \\
\text { (mmag) }\end{array}$ \\
\hline \multicolumn{5}{|c|}{ Ren ID 49650 (KIC 11445913, 1SWASP J190540.61+491820.7) } \\
\hline$f_{1}$ & $31.5577 \pm 0.0003$ & 2.8 & $31.5577 \pm 0.0001$ & $3.2 \pm 0.1$ \\
\hline$f_{2}$ & $25.3799 \pm 0.0007$ & 1.1 & $25.3769 \pm 0.0001$ & $1.2 \pm 0.1$ \\
\hline$f_{3}$ & $22.1307 \pm 0.0009$ & 0.8 & $22.1306 \pm$ & $1.0 \pm$ \\
\hline$f_{4}$ & $37.8182 \pm 0.0011$ & 0.6 & & \\
\hline$f_{5}$ & $29.7394 \pm 0.0012$ & 0.6 & & \\
\hline \multicolumn{5}{|c|}{ Ren ID 50230 (KIC 9764965, 1SWASP J191724.91+463535.2) } \\
\hline$f_{1}$ & $27.1777 \pm 0.0001$ & 1.1 & $27.1778 \pm 0.0001$ & $1.2 \pm 0.1$ \\
\hline$f_{2}$ & $21.3819 \pm$ & 0.6 & 001 & $0.9 \pm$ \\
\hline$f_{3}$ & $31.9895 \pm 0.0002$ & 0.4 & $31.9902 \pm 0.0001$ & $0.9 \pm 0.1$ \\
\hline & $19.9579 \pm 0.0004$ & 0.2 & & \\
\hline \multicolumn{5}{|c|}{ Ren ID 50420 (KIC 8881697, 1SWASP J192136.03+450706.8) } \\
\hline$f_{1}$ & $16.5567 \pm 0.0003$ & 1.9 & $16.5565 \pm 0.0005$ & $2.1 \pm 0.1$ \\
\hline$f_{2}$ & 32. & 1 & 6 & 1.6 \\
\hline$f_{3}$ & $25.2105 \pm$ & 1.2 & 08 & $1.2=$ \\
\hline$f_{4}$ & 30.01 & 1.1 & & 1.1 \\
\hline$f_{5}$ & $34.3647 \pm 0.0007$ & 0.9 & $34.3661 \pm 0.0009$ & $1.0 \pm$ \\
\hline$f_{6}$ & $30.6537 \pm 0.0008$ & 0.9 & $30.6569 \pm 0.0010$ & $0.9 \pm$ \\
\hline$f_{7}$ & $28.8044 \pm 0.0009$ & 0.7 & $27.8049 \pm 0.0011$ & $0.8 \pm 0$ \\
\hline$f_{8}$ & $34.0106 \pm 0.0009$ & 0.7 & & \\
\hline$f_{9}$ & $27.4073 \pm 0.0010$ & 0.7 & & \\
\hline & $16.0119 \pm 0.0013$ & 0.5 & & \\
\hline \multicolumn{5}{|c|}{ Ren ID 50670 (KIC 11402951, 1SWASP J192732.81+491523.5) } \\
\hline$f_{1}$ & $23.8493 \pm 0.0004$ & 1.3 & $23.8464 \pm 0.0008$ & $1.4 \pm 0.2$ \\
\hline$f_{2}$ & $23.2770 \pm 0.0004$ & 1.1 & $23.2790 \pm 0.0008$ & $1.4 \pm 0.2$ \\
\hline$f_{3}$ & $27.4616 \pm 0.0007$ & 0.7 & $27.4643 \pm 0.0012$ & $1.0 \pm 0.2$ \\
\hline$f_{4}$ & $15.1001 \pm 0.0007$ & 0.7 & & \\
\hline$f_{4}$ & $14.4967 \pm 0.0009$ & 0.5 & & \\
\hline
\end{tabular}

Notes. ${ }^{(a)}$ Uncertainties on Kepler Amplitudes are all $<0.05$ mmag.

\section{Discussion}

The pulsating Am stars (see Fig. 3) are concentrated within the fundamental radial mode red and blue edges of Dupret et al. (2005). This is in agreement with that found by Balona et al. (2011) for Am stars within the Kepler field. These studies show that pulsating Am stars are concentrated in the cooler region of the instability strip. Hot Am stars do not appear to pulsate at the precision of the Kepler data.

The standard interpretation of the Am phenomenon is that atomic diffusion - radiative levitation and gravitational settling in the outer stellar envelope gives rise to the observed atmospheric abundance anomalies. For a typical mid-A star, $T_{\text {eff }} \sim$ $8000 \mathrm{~K}$, there are two thin convection zones in the outer envelope. The atmosphere itself is a convection zone a few thousand $\mathrm{km}$ thick where ionisation of $\mathrm{H}$ drives the convection. Deeper in the atmosphere, at $T \sim 50000 \mathrm{~K}$, the ionisation of He II also creates a thin convection zone, where the $\kappa$-mechanism drives $\delta$ Sct 
KIC011445913

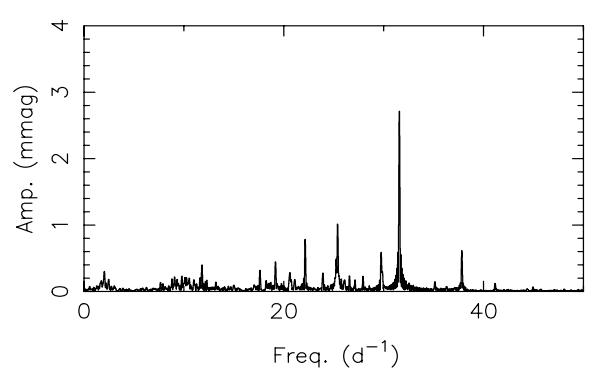

KIC009764965

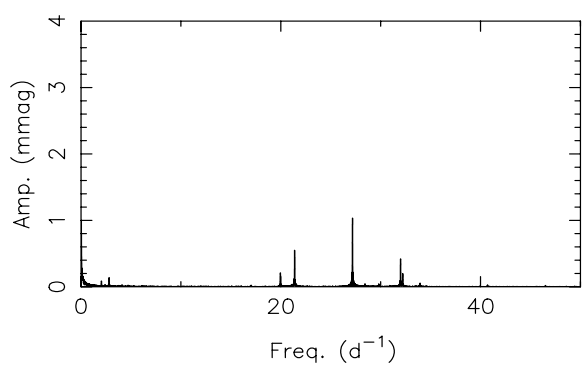

KIC008881697

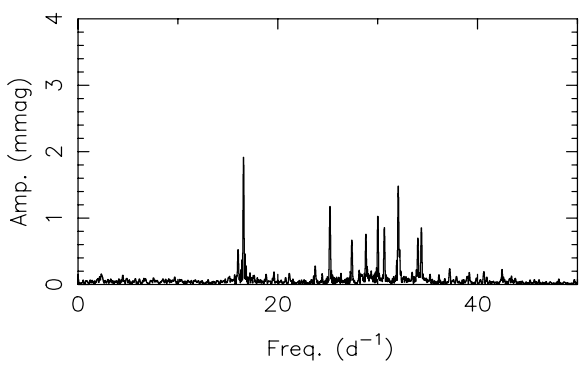

KIC011402951

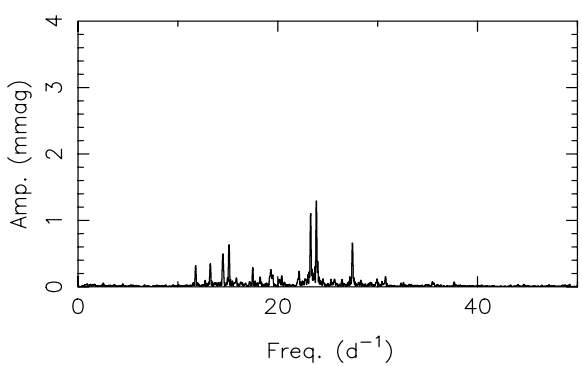

Ren ID 49650 (1SWASPJ190540.61+491820.7)

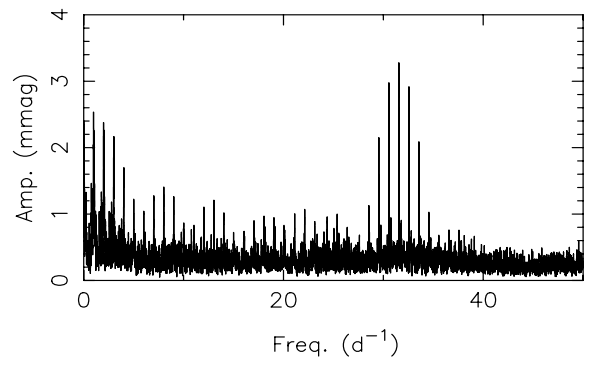

Ren ID 50230 (1SWASPJ191724.91+463535.2)

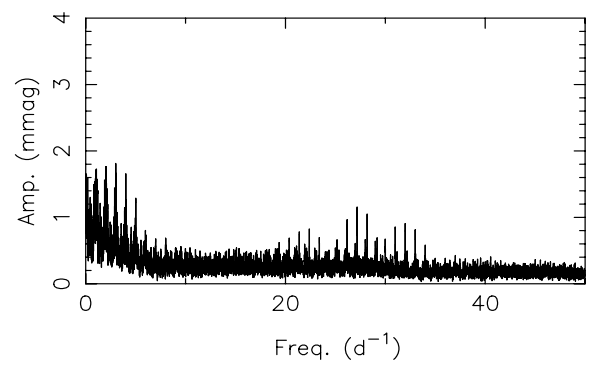

Ren ID 50420 (1SWASPJ192136.03+450706.8)

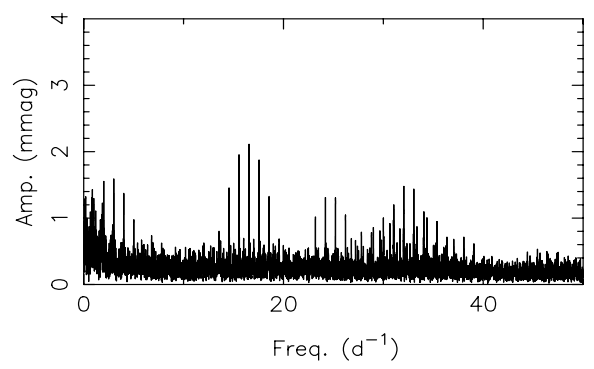

Ren ID 50670 (1SWASPJ192732.81+491523.5)

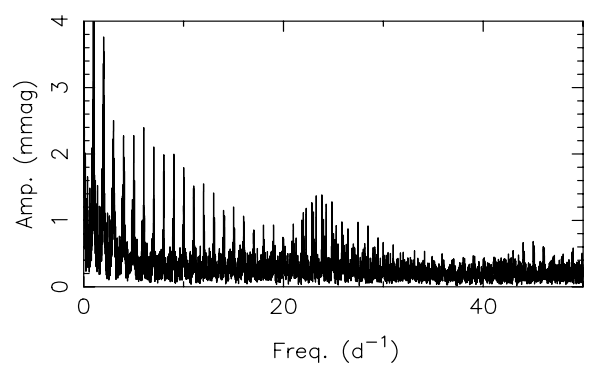

Fig. 2. Comparison between the PERIOD04 periodograms from Kepler (left) and SuperWASP (right) for four Am stars with pulsations detected by SuperWASP (see Table 3 for details of frequencies identified).

pulsation. It has long been clear that some Am stars and related types do pulsate, particularly the marginal Am stars (labelled spectroscopically as Am: stars), the evolved Am stars ( $\delta$ Del or $\rho$ Pup stars), and some more extreme cases, such as HD 188136 (Kurtz 1980; Wegner 1981) and HD 40765 (Kurtz et al. 1995).

The pulsation modes that we observe in Am stars are low radial order, low spherical degree $p$ modes. The surface of the star is an anti-node. With the low radial order, the vertical wavelength is long compared to the depth of the envelope above the He II ionisation zone. With the decrease in density with height in the atmosphere, conservation of kinetic energy density means that the pulsation amplitude increases with height in the atmosphere, or conversely, decreases with depth.
In Am stars, the microturbulence velocity is also peculiar, as it is generally much higher than that of chemically normal stars. This high microturbulence arises from large velocity fields in the stellar atmosphere (Landstreet 1998), which are even supersonic for some Am stars. We do not really know what causes these large velocity fields to develop exclusively in Am stars and how chemical peculiarities and velocity fields coexist. The results shown by Landstreet et al. (2009) suggest that there is a connection between $T_{\text {eff }}$ and the velocity fields, peaking at around $T_{\text {eff }} \sim 8000 \mathrm{~K}$, although we do not know what happens for cooler Am stars.

Atomic diffusion occurs in the radiative zone below the turbulent outer convective layer, which is far below the observable 


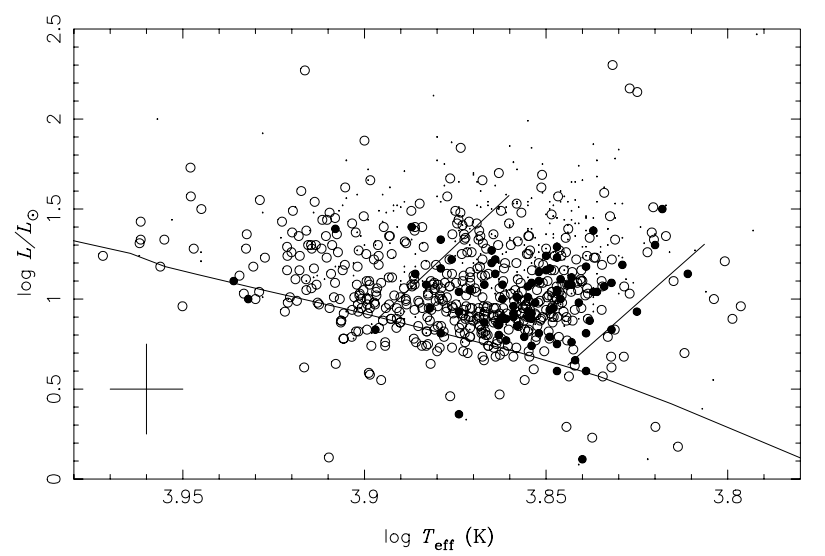

Fig. 3. HR diagram showing the location of Am stars. The filled circles are the Am stars which were found to pulsate, while the open circles are the Am stars which were not found to pulsate. The solid lines indicate the location of the ZAMS and the fundamental radial mode red and blue edges of the instability strip (Dupret et al. 2005). The large cross indicates the typical uncertainties in $\log T_{\text {eff }}$ and $\log L$. The dots are the $\delta$ Sct stars from the catalogue of Rodríguez et al. (2000).

atmosphere. In this radiative layer there must be no turbulence at the diffusion velocity, which is of the order of $10^{-4}-1 \mathrm{~cm} \mathrm{~s}^{-1}$. The photometric amplitudes found in Am stars are consistent with atmospheric pulsation radial velocity amplitudes of a few $\mathrm{km} \mathrm{s}^{-1}$. Taking into account the decrease in pulsation amplitude with depth - largely because of the increase in density, but also because of the radial wave function - the pulsation velocity in the radiative layer where atomic diffusion is most important in Am stars is still of the order of a $\mathrm{km} \mathrm{s}^{-1}$. With such pulsations in a layer where atomic diffusion is operating at sub-cm s${ }^{-1}$ velocities, it must be that the pulsation is laminar; i.e., producing no turbulence at the sub-cm s${ }^{-1}$ level.

With the results from the Kepler mission (Balona et al. 2011) and now our results from SuperWASP we conclude that the loss of helium by gravitational settling from the He II ionisation zone reduces driving, but does not suppress it entirely. Thus Am stars can pulsate as $\delta$ Sct stars, but typically with relatively low amplitudes compared to normal abundance $\delta$ Sct stars. Some Am stars show no pulsation whatsoever at Kepler $\mu$ mag precision. It has yet to be shown whether this lack of pulsation can also occur in the more rapidly rotating normal abundance stars in the $\delta$ Sct instability strip. Study of this question is in progress with Kepler data. As was concluded for the individual cases of HD 188136 and HD 40765, we may now state in general: in Am stars the pulsation must be laminar, not generating turbulence to mix away the observed effects of atomic diffusion in the outer atmosphere.

The Fm $\delta$ Del subclass are evolved Am stars above the mainsequence, many of which have been found to show variability (Kurtz 1976). Not unexpectedly, many stars classed as Fm $\delta$ Del are found to be pulsating in the WASP data, but clearly not all. Of the 227 Am stars that we found to be pulsating 55 are classed as Fm $\delta$ Del: $24 \%$ of the Am stars found to pulsate. This compares to a total of $186 \mathrm{Fm} \delta$ Del stars out of the 1620 Am stars investigated using WASP data, around $11 \%$ of the sample. Therefore, $30 \%$ of the $\operatorname{Fm} \delta$ Del stars have been found to pulsate, compared to just $12 \%$ of other Am stars. Thus pulsation amplitude either grows in Am stars as they evolve, or some non-pulsating Am stars begin pulsating as they move off the main sequence. This is likely to be a consequence of the driving region moving deeper

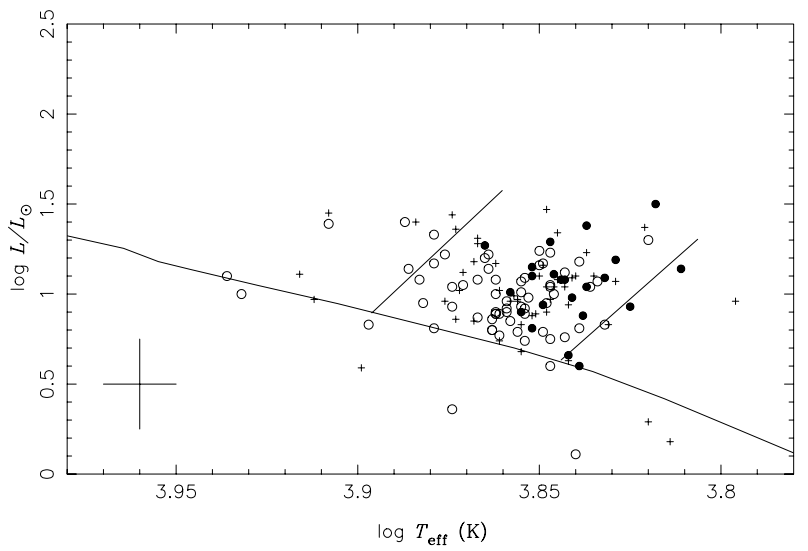

Fig. 4. Location of the pulsating Am stars in the HR diagram. The circles are pulsating Am stars, with the filled circles indicating those with spectral classification noted as $\delta$ Del. The crosses are the Fm $\delta$ Del stars which were not found to pulsate. The solid lines indicate the location of the ZAMS and the fundamental radial mode red and blue edges of the instability strip (Dupret et al. 2005). The large cross indicates the typical uncertainties in $\log T_{\text {eff }}$ and $\log L$.

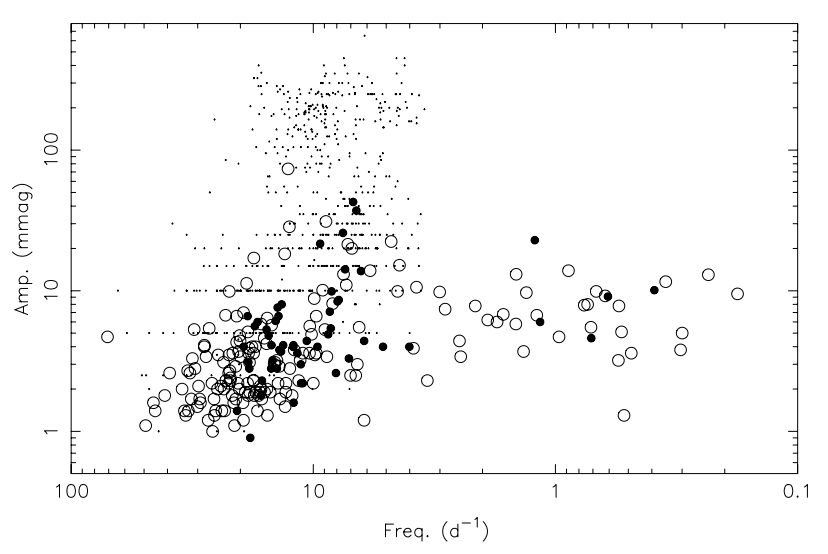

Fig. 5. Frequency-amplitude diagram for pulsating Am stars shown as circles, with filled circles indicating those with spectral classification noted as $\delta$ Del. Note that in multi-periodic systems only the frequency of the highest amplitude is shown, as given in Table 1 . The dots are the $\delta$ Sct stars from the catalogue of Rodríguez et al. (2000).

into the star where the helium abundance is higher than in the main sequence He II ionisation zone (see Turcotte et al. 2000 for theoretical discussion).

The location of the pulsating Fm $\delta$ Del stars in the HR diagram is shown in Fig. 4. There is a tendency for the pulsating $\operatorname{Fm} \delta$ Del stars to be located toward the cooler (and/or) slightly more evolved parts of the instability strip, whereas the non-pulsating $\operatorname{Fm} \delta$ Del stars are distributed more uniformly. The frequency-amplitude diagram (Fig. 5) shows that the Fm $\delta$ Del stars occupy the same regions as the other Am stars, but with an absence of high-frequency $\left(\gtrsim 20 \mathrm{~d}^{-1}\right)$ pulsations; this is not surprising, given that they are cooler and more evolved than average $\delta$ Sct stars.

Several factors are thought to play a role in the development of pulsating Am stars, but stellar rotation is probably one of the most important. Charbonneau \& Michaud (1991) showed that Am chemical peculiarity develops in stars that rotate slower than 
Table 4. The number of pulsating Am stars and percentage in each of the four pulsation classes as defined by Grigahcène et al. (2010).

\begin{tabular}{lcc}
\hline \hline Pulsation Class & Number & Percentage \\
\hline$\delta$ Sct & 169 & 75 \\
$\delta$ Sct $/ \gamma$ Dor & 23 & 10 \\
$\gamma$ Dor & 30 & 13 \\
$\gamma$ Dor $/ \delta$ Sct & 5 & 2 \\
\hline
\end{tabular}

$90 \mathrm{~km} \mathrm{~s}^{-1}$ and that the He II ionisation zone deepens with decreasing rotation. This was later confirmed by more advanced diffusion model calculations by Talon et al. (2006) and observationally by Fossati et al. (2008), who found a correlation between Am chemical peculiarities and $v \sin i$ in Am stars belonging to the Praesepe open cluster. The vast majority of the Am stars already known to pulsate have a rather large $v \sin i$, between 40 and $90 \mathrm{~km} \mathrm{~s}^{-1}$, thus avoiding the He II ionisation zone sinking too deep into the star and therefore allowing the development of pulsation driven by the $\kappa$-mechanism. On the other hand, for the very slowly rotating pulsating Am stars, the pulsation could be laminar. It is therefore likely there are two different mechanisms driving pulsation in Am stars.

Our results show a wide variety of pulsations, from singly periodic to complex multiperiodic, and also some examples of what appear to be hybrid $\gamma$ Dor $/ \delta$ Sct pulsators. This is similar to the range of behaviour seen in normal abundance $\delta$ Sct stars, as can be seen in the study of Kepler data by Grigahcène et al. (2010). Those authors reclassified pulsation types with the following scheme:

$\delta$ Sct: $\quad$ frequencies above $5 \mathrm{~d}^{-1}$;

$\delta \mathrm{Sct} / \gamma$ Dor hybrid: most frequencies above $5 \mathrm{~d}^{-1}$, but some low frequencies present;

$\gamma$ Dor: $\quad$ frequencies lower than $5 \mathrm{~d}^{-1}$;

$\gamma$ Dor $/ \delta$ Sct hybrid: most frequencies lower than $5 \mathrm{~d}^{-1}$, but some high frequencies present.

Our results are summarized in Table 4 and the individual classes for each star are given in Table 1. The majority of the pulsators we found are $\delta$ Sct stars, with the remaining quarter split between $\gamma$ Dor stars and mostly $\delta$ Sct $/ \gamma$ Dor hybrids. Given that the SuperWASP data are affected by daily aliases and systematics at low frequencies, the true number of stars with $\gamma$ Dor pulsations may indeed be higher. However, given that Am stars are thought to be members of binary systems and tidal effects slow the stellar rotation rate, it is possible that some of the lowfrequency signatures found in the SuperWASP data are due to ellipsoidal effects in close binaries. Assuming a rotation limit of $v \sin i \lesssim 120 \mathrm{~km} \mathrm{~s}^{-1}$ for an Am star and a radius of $1.5 R_{\odot}$, the shortest period for a binary system containing a tidallysynchronised Am star is $\sim 0.6 \mathrm{~d}$. Close binary systems with dissimilar components have two maxima and minima per orbital period, and this value dominates over the orbital value in periodograms. Hence, frequencies $\$ 3.3 \mathrm{~d}^{-1}$ may have arisen due to ellipsoidal variations in close binaries. Thus, we caution that some of the stars presented in Table 1 could have erroneously been classified as having $\gamma$ Dor pulsations. In addition, it is possible that long-period pulsations in close binaries could be tidally excited (Handler et al. 2002).

It is clear from examination of the Kepler data set that the $\delta$ Sct stars show frequencies ranging from nearly zero $\mathrm{d}^{-1}$ up to $100 \mathrm{~d}^{-1}$; some stars even show the full range, including frequencies between the gmode and p mode ranges seen in models. These intermediate frequencies are unexplained at present. It is clear that the $\delta$ Sct stars are complex pulsators that show g modes, $\mathrm{p}$ modes, mixed modes and many nonlinear cross terms. Whether there are differences between abnormal abundance, slowly rotating Am stars that are $\delta$ Sct stars and the more rapidly rotating, normal abundance $\delta$ Sct stars is yet to be determined. The objects we present here from SuperWASP greatly increases the number of pulsating Am stars for statistical study of this question.

Acknowledgements. The WASP project is funded and operated by Queen's University Belfast, the Universities of Keele, St. Andrews and Leicester, the Open University, the Isaac Newton Group, the Instituto de Astrofisica de Canarias, the South African Astronomical Observatory and by STFC. This research has made use of the SIMBAD database, operated at CDS, Strasbourg, France. Some of the data presented in this paper were obtained from the Multimission Archive at the Space Telescope Science Institute (MAST). STScI is operated by the Association of Universities for Research in Astronomy, Inc., under NASA contract NAS5-26555. Support for MAST for non-HST data is provided by the NASA Office of Space Science via grant NNX09AF08G and by other grants and contracts.

\section{References}

\section{Abt, H. A. 2009, AJ, 138, 28}

Aerts, C., Christensen-Dalsgaard, J., \& Kurtz, D. W. 2010, Asteroseismology (Heidelberg: Springer)

Balona, L., Ripepi, V., Catanzaro, G., et al. 2011, MNRAS, 414, 792

Breger, M., Stich, J., Garrido, R., et al. 1993, A\&A, 271, 482

Butters, O. W., West, R. G., Anderson, D. R., et al. 2010, A\&A, 520, L10

Catanzaro, G., Ripepi, V., Bernabei, S., et al. 2011, MNRAS, 411, 1167

Charbonneau, P., \& Michaud, G. 1991, ApJ, 370, 693

Dupret, M.-A., Grigahcène, A., Garrido, R., Gabriel, M., \& Scuflaire, R. 2005, A\&A, 435, 927

Fossati, L., Bagnulo, S., Landstreet, J., et al. 2008, A\&A, 483, 891

Gilliland, R. L., Brown, T. M., Christensen-Dalsgaard, J., et al. 2010, PASP, 122, 131

Grigahcène, A., Antoci, V., Balona, L., et al. 2010, ApJ, 713, L192

Handler, G., Balona, L. A., Shobbrook, R. R., et al. 2002, MNRAS, 333, 262

Hauck, B., \& Mermilliod, M. 1998, A\&AS, 129, 431

Henry, G. W., \& Fekel, F. C. 2005, AJ, 129, 2026

Horne, J. H., \& Baliunas, S. L. 1986, ApJ, 302, 757

Joshi, S., Mary, D. L., Martinez, P., et al. 2006, A\&A, 455, 303

Joshi, S., Mary, D. L., Chakradhari, N. K., Tiwari, S. K., \& Billaud, C. 2009, A\&A, 507, 1763

Künzli, M., North, P., Kurucz, R. L., \& Nicolet, B. 1997, A\&AS, 122, 51

Kurtz, D. W. 1976, ApJS, 32, 651

Kurtz, D. W. 1978, ApJ, 221, 869

Kurtz, D. W. 1980, MNRAS, 193, 29

Kurtz, D. W. 1989, MNRAS, 238, 1077

Kurtz, D. W., \& Martinez, P. 2000, Baltic Astron., 9, 253

Kurtz, D. W., Garrison, R. F., Koen, C., Hofmann, G. F., \& Viranna, N. B. 1995, MNRAS, 276, 199

Landstreet, J. D. 1998, A\&A, 338, 1041

Landstreet, J. D., Kupka, F., Ford, H. A., et al. 2009, A\&A, 503, 973

Lenz, P., \& Breger, M. 2005, CoAst, 146, 53

Montgomery, M. H., \& O'Donoghue, D. 1999, DSSN, 13, 28

Moon, T. T. 1985, Commun. Univ. London Obs., 78

Rufener, F. 1998, Observations in the Geneva Photometric System 4 [CDS Catalog II/169/]

Pollacco, D. L., Skillen, I., Collier Cameron, A., et al. 2006, PASP, 118, 1407

Press, W. H., \& Rybicki, G. B. 1989, ApJ, 338, 277

Press, W. H., Teukolsky, S. A., Vetterling, W. T., \& Flannery, B. P. 1992,

Numerical recipes in FORTRAN, 2nd ed. (Cambridge: Cambridge Univ. Press)

Renson, P., \& Manfroid, J. 2009, A\&A, 498, 961

Rodríguez, E., López-González, M. J., \& López de Coca, P. 2002, A\&AS, 144, 469

Smalley, B., \& Kupka, F. 1997, A\&A, 328, 349

Talon, S., Richard, O., \& Michaud, G. 2006, ApJ, 645, 634

Tamuz, O., Mazeh, T., \& Zucker, S. 2005, MNRAS, 356, 1466

Torres, G., Andersen, J., \& Giménez, A. 2010, A\&AR, 18, 67

Turcotte, S., Richer, J., Michaud, G., \& Christensen-Dalsgaard, J. 2000, A\&A, 360,603

van Leeuwen, F. 2007, A\&A, 474, 653

Wegner, G. 1981, ApJ, 247, 969 
B. Smalley et al.: SuperWASP observations of pulsating Am stars

Ren ID 10 (1SWASPJ000624.29+343624.8)

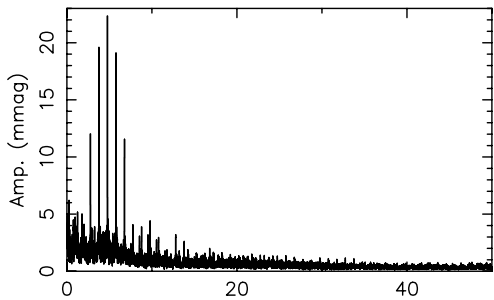

Freq. $\left(d^{-1}\right)$

Frequency $4.7672081 d^{-1}$

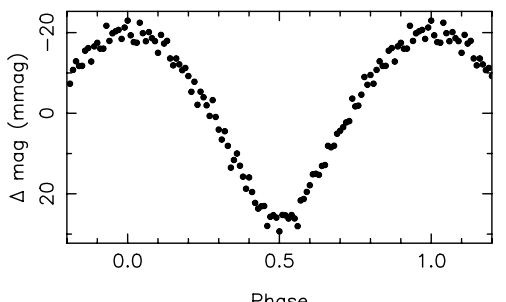

Ren ID 140 (1SWASPJ001334.00-293432.5)

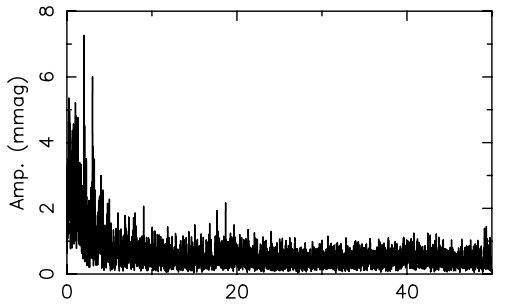

Freq. $\left(d^{-1}\right)$

Frequency $18.668438 d^{-1}$

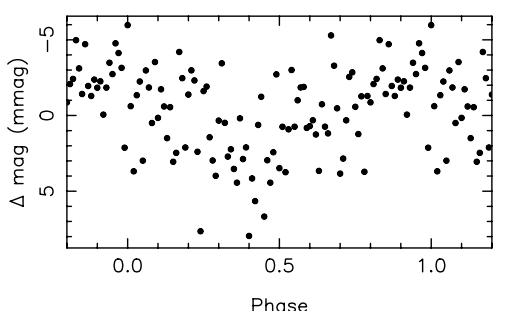

Ren ID 500 (1SWASPJ002512.87+412039.6)

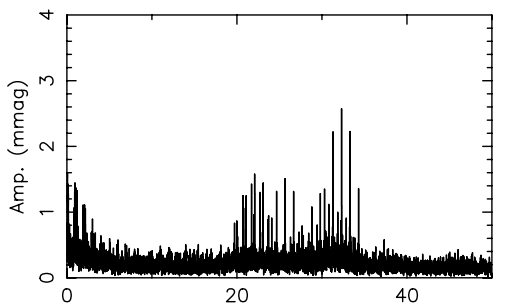

Freq. $\left(d^{-1}\right)$

Frequency $32.3129768 d^{-1}$

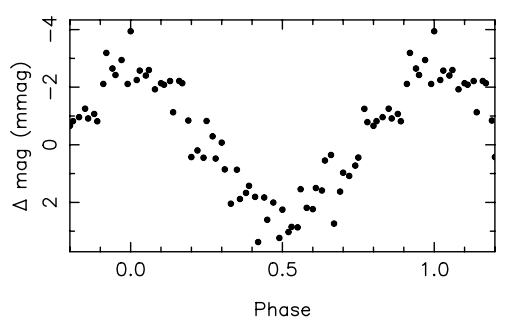

Ren ID 110 (1SWASPJ001128.62-261511.7)

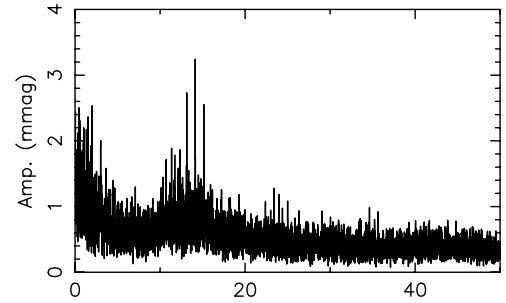

Freq. $\left(d^{-1}\right)$

Frequency $14.1368027 \mathrm{~d}^{-1}$

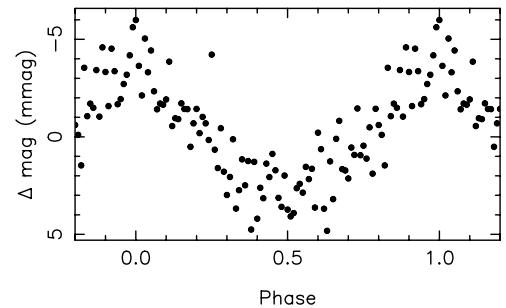

Ren ID 210 (1SWASPJ001507.96-290023.0)

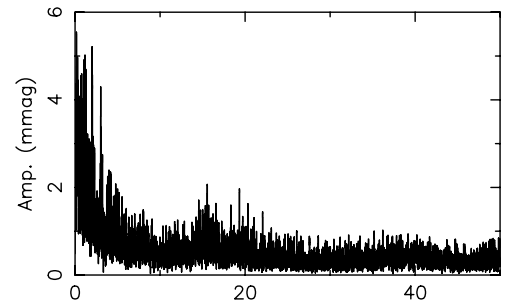

Freq. $\left(d^{-1}\right)$

Frequency $15.5490952 d^{-1}$

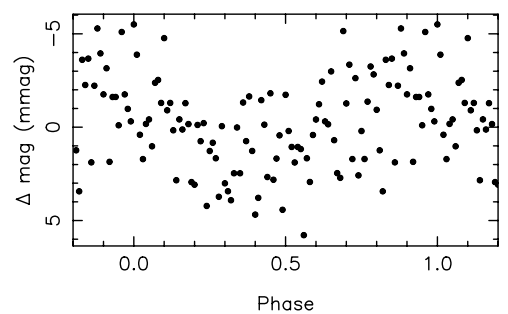

Ren ID 1233 (1SWASPJ004809.56-031824.5)

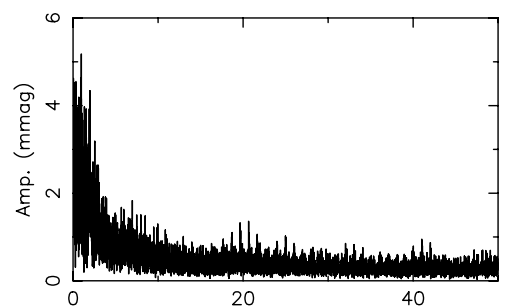

Freq. $\left(d^{-1}\right)$

Frequency $20.6497288 d^{-1}$

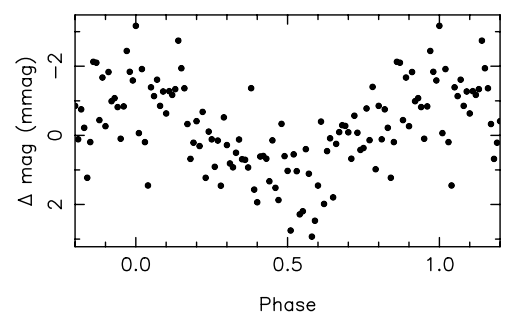

Ren ID 113 (1SWASPJ001140.67+000719.4)

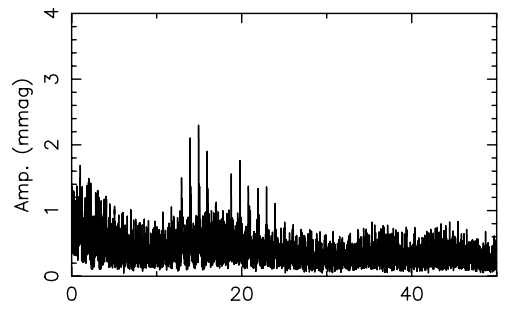

Freq. $\left(d^{-1}\right)$

Frequency $14.926815 d^{-1}$

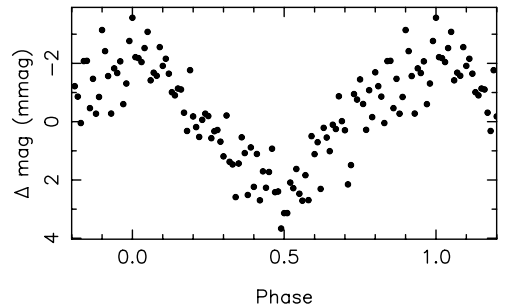

Ren ID 355 (1SWASPJ002049.76-005215.9)

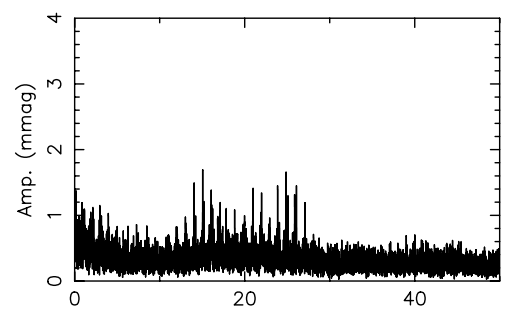

Freq. $\left(d^{-1}\right)$

Frequency $15.0600405 d^{-1}$

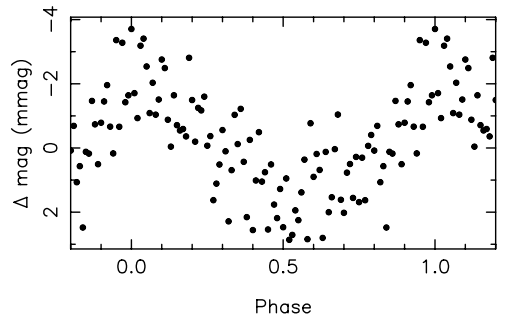

Ren ID 1790 (1SWASPJ011134.98-052024.7)

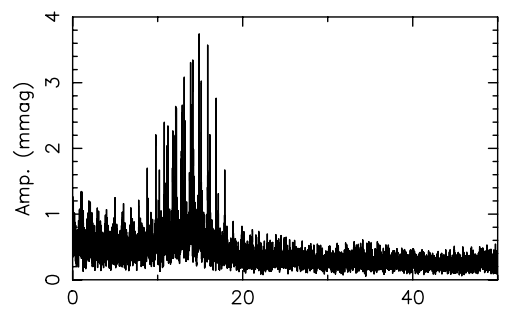

Freq. $\left(d^{-1}\right)$

Frequency $14.8754635 \mathrm{~d}^{-1}$

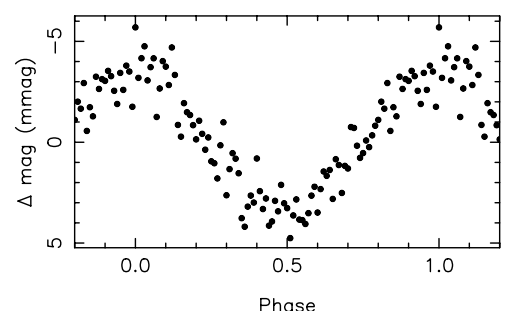

Fig. 1. SuperWASP periodograms for the pulsating Am stars (top) and corresponding lightcurves folded on the principal frequency (below). The lightcurves have been binned in 0.01 phase steps and phase 0.0 corresponds to the time of maximum light. 


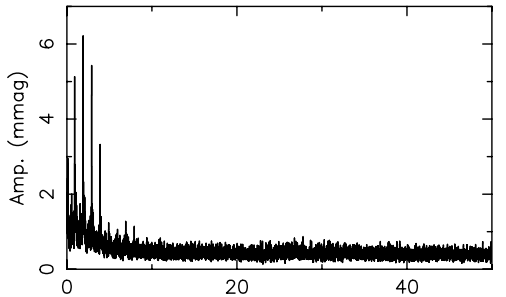

Freq. $\left(d^{-1}\right)$

Frequency $1.9040935 d^{-1}$

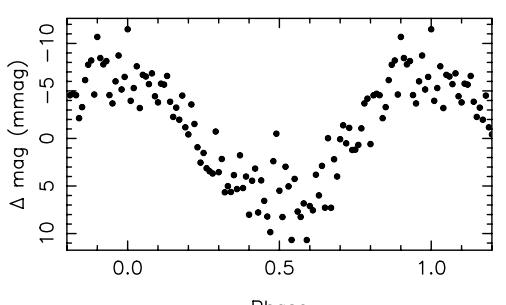

Ren ID 2060 (1SWASPJ012334.25-130234.0)

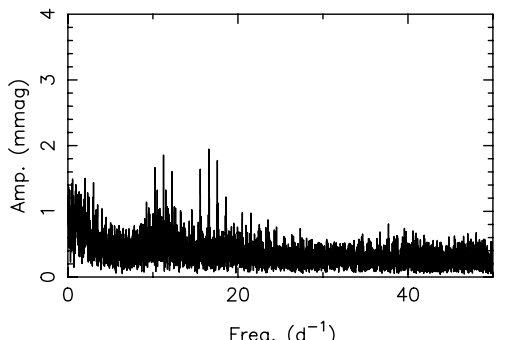

Frequency $16.5740757 d^{-1}$

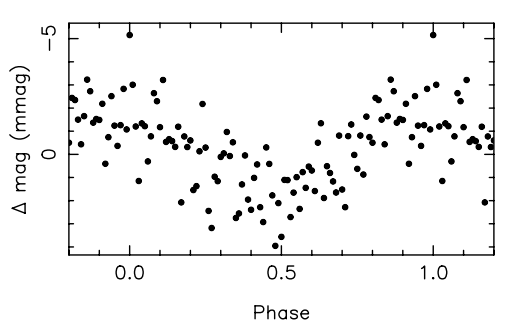

Ren ID 2720 (1SWASPJ014849.55+585832.3)

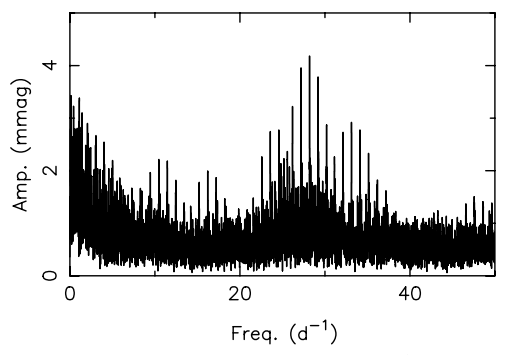

Frequency $28.191164 d^{-1}$

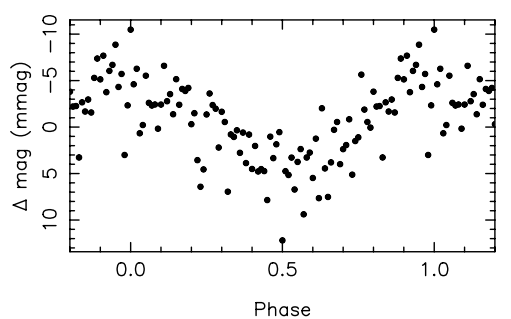

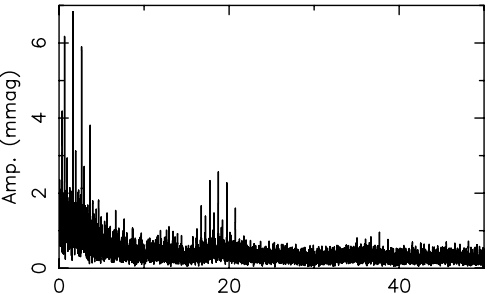

Freq. $\left(d^{-1}\right)$

Frequency $1.64204299 d^{-1}$

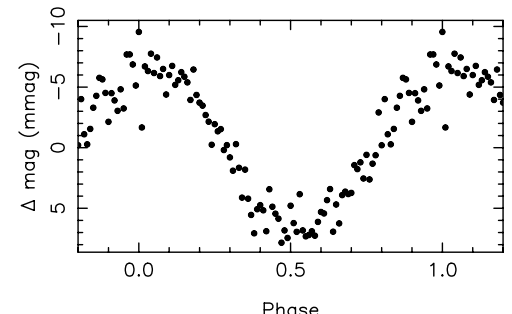

Ren ID 2340 (1SWASPJ013405.68-112212.9)

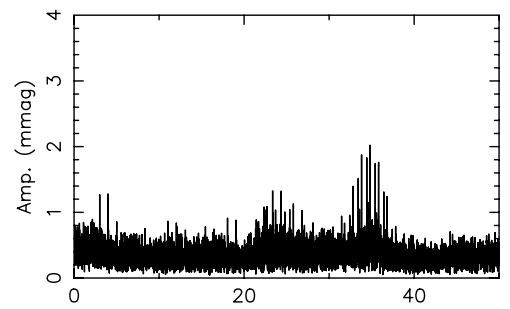

Freq. $\left(d^{-1}\right)$

Frequency $34.8219414 d^{-1}$

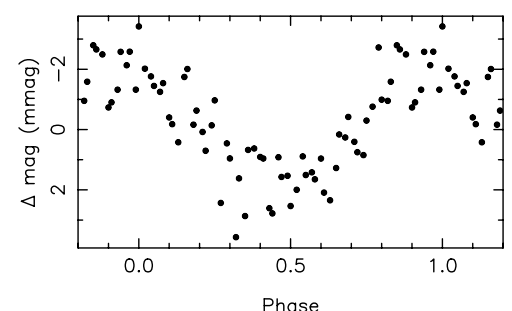

Ren ID 2920 (1SWASPJ015209.74-361429.5)

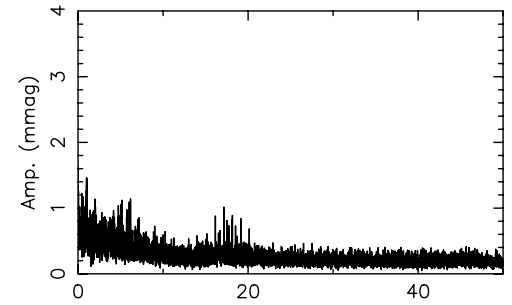

Freq. $\left(d^{-1}\right)$

Frequency $6.1678896 d^{-1}$

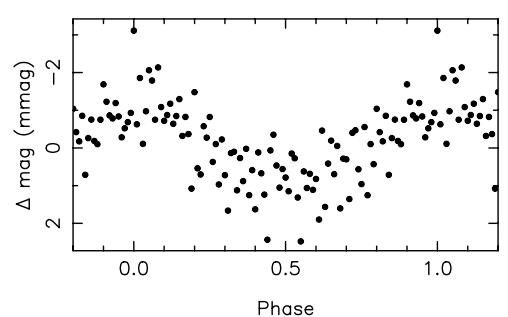

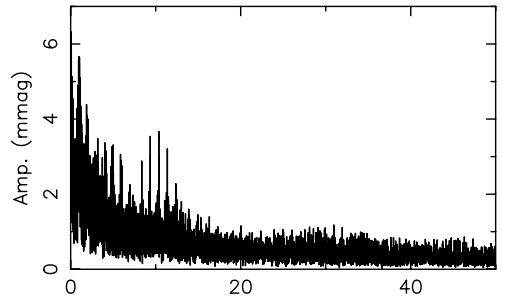

Freq. $\left(d^{-1}\right)$

Frequency $10.3662443 d^{-1}$

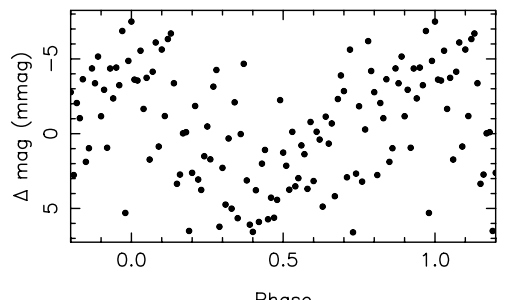

Ren ID 2370 (1SWASPJ013426.15-174353.2)

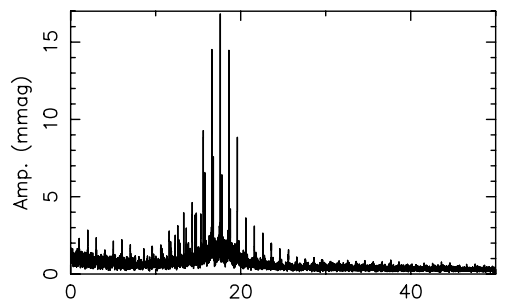

Freq. $\left(d^{-1}\right)$

Frequency $17.6028271 d^{-1}$

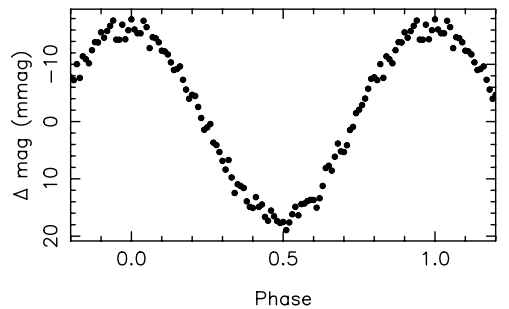

Ren ID 3013 (1SWASPJ015608.96+373952.7)

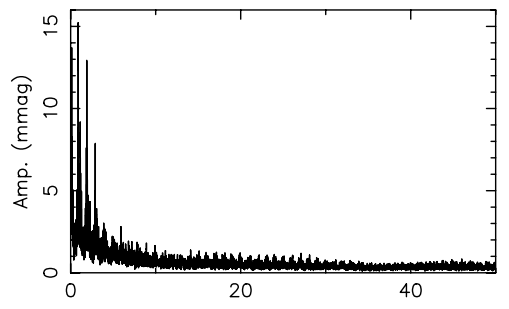

Freq. $\left(d^{-1}\right)$

Frequency $0.883810818 d^{-1}$

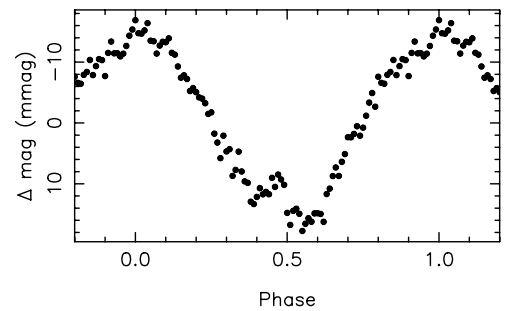

Fig. 1. continued. 
B. Smalley et al.: SuperWASP observations of pulsating Am stars

Ren ID 3340 (1SWASPJ020549.28-415541.9)

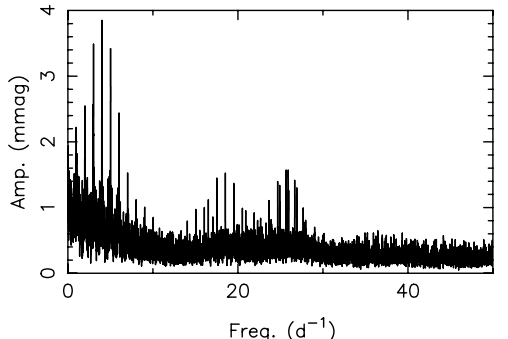

Frequency $4.01150274 \mathrm{~d}^{-1}$

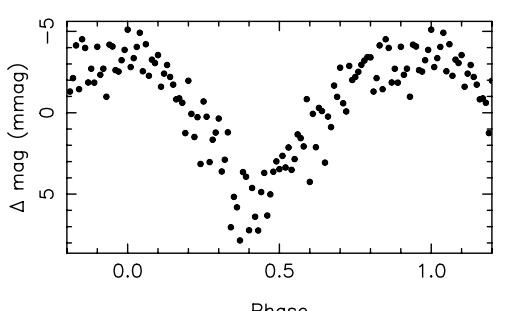

Ren ID 3655 (1SWASPJ021958.53-190341.7)

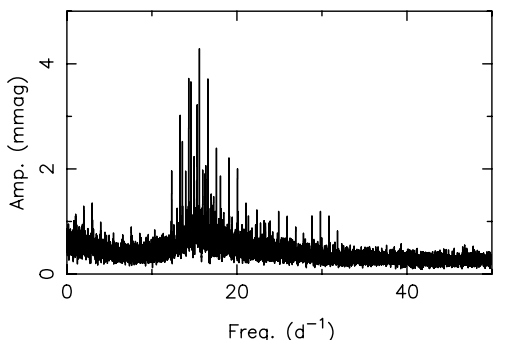

Frequency $15.5854826 d^{-1}$

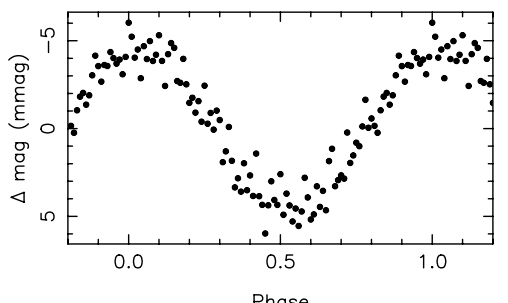

Ren ID 4885 (1SWASPJ031024.31-132232.5)

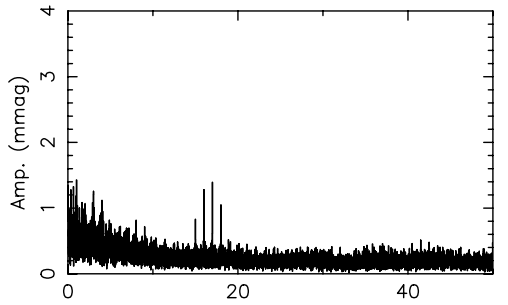

Freq. $\left(d^{-1}\right)$

Frequency $16.9993057 \mathrm{~d}^{-1}$

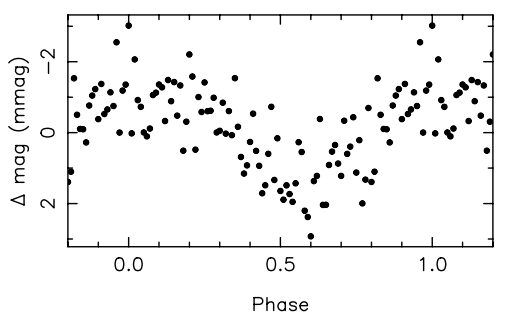

Ren ID 3378 (1SWASPJ020902.32+393532.2)

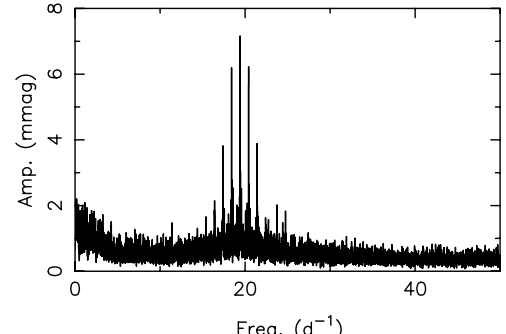

Frequency $19.408989 d^{-1}$

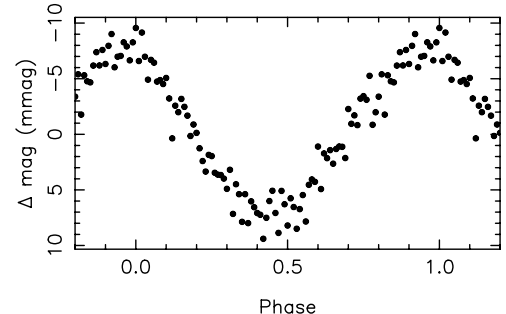

Ren ID 4413 (1SWASPJ024939.64+010407.1)

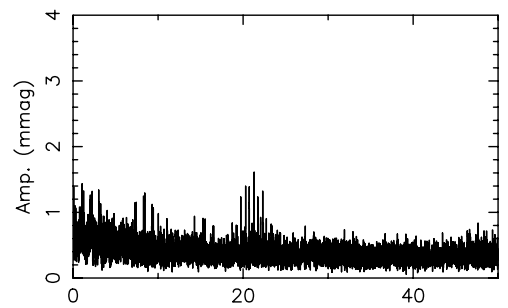

Freq. $\left(d^{-1}\right)$

Frequency $21.3019028 d^{-1}$

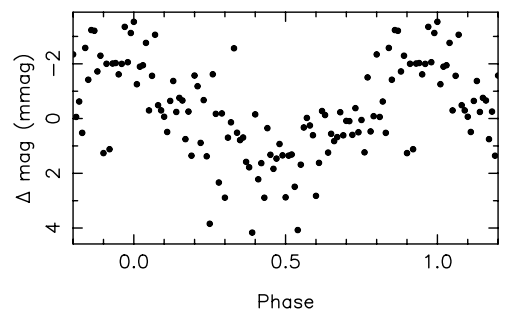

Ren ID 5055 (1SWASPJ031531.64-163317.6)

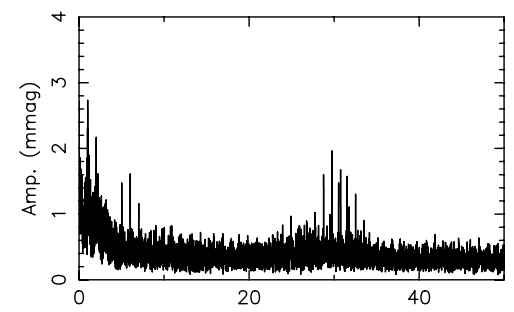

Freq. $\left(d^{-1}\right)$

Frequency $29.7633915 d^{-1}$

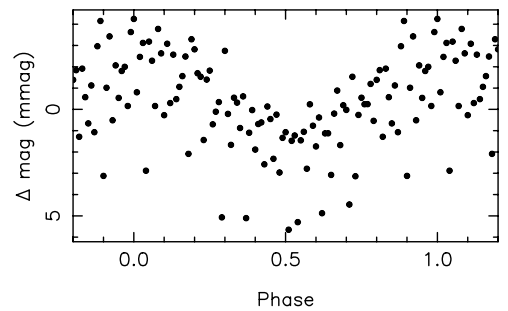

Ren ID 3550 (1SWASPJ021345.99-235824.7)

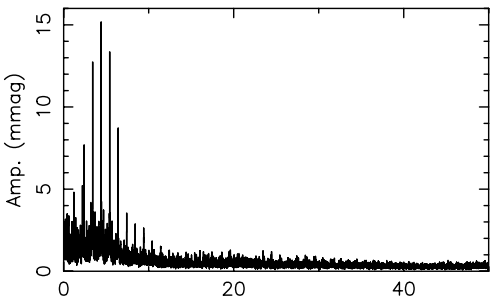

Freq. $\left(d^{-1}\right)$

Frequency $4.40780878 d^{-1}$

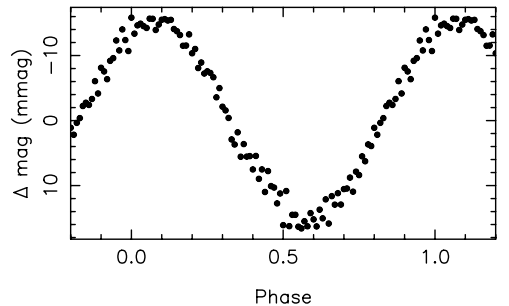

Ren ID 4793 (1SWASPJ030413.47-082752.4)

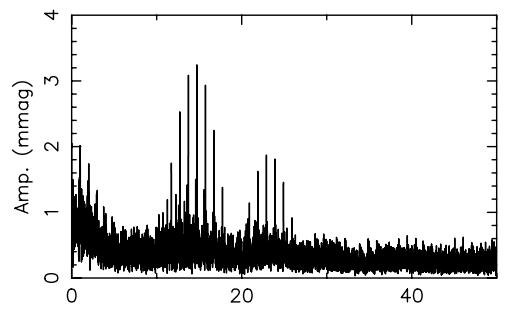

Freq. $\left(d^{-1}\right)$

Frequency $14.7305946 d^{-1}$

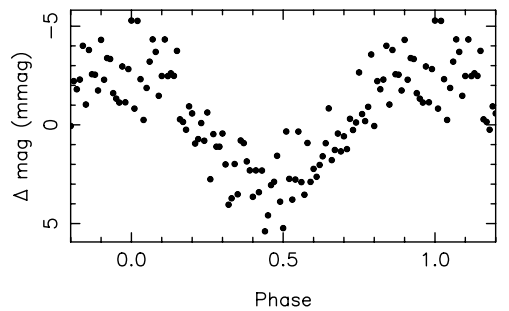

Ren ID 6044 (1SWASPJ034512.81-215834.5)

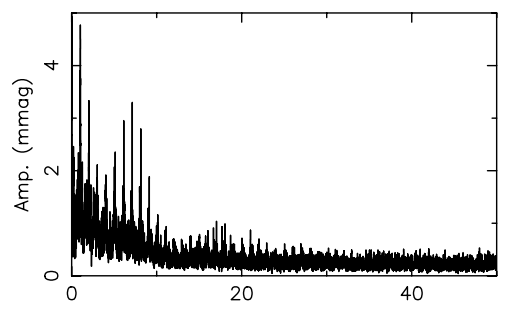

Freq. $\left(d^{-1}\right)$

Frequency $7.1267724 d^{-1}$

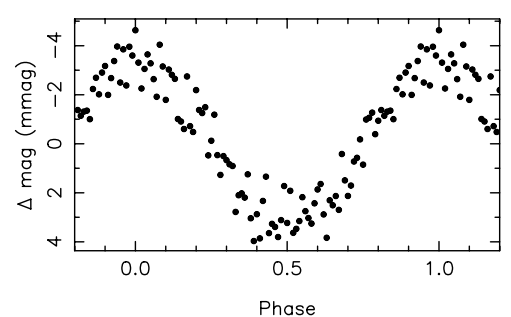

Fig. 1. continued. 
Ren ID 6083 (1SWASPJ035020.03+582454.8)

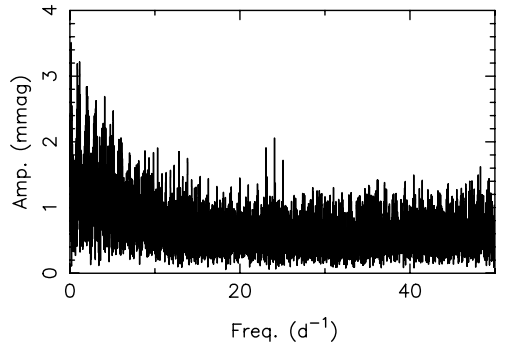

Frequency $24.0685692 \mathrm{~d}^{-1}$

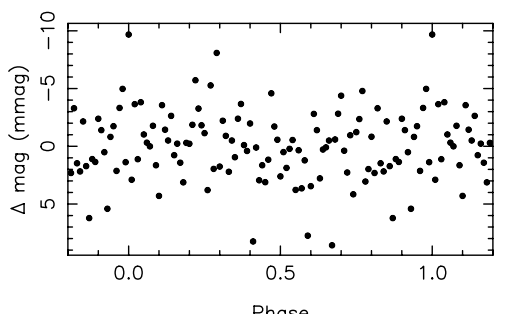

Ren ID 6368 (1SWASPJ035734.08-053400.8)

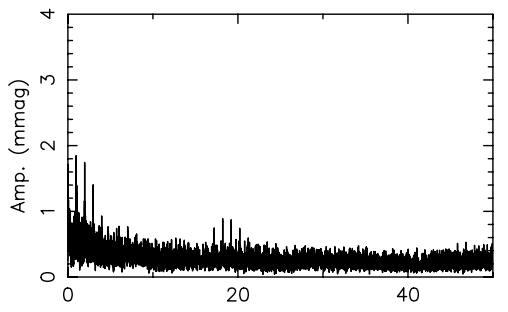

Freq. $\left(d^{-1}\right)$

Frequency $18.208786 d^{-1}$

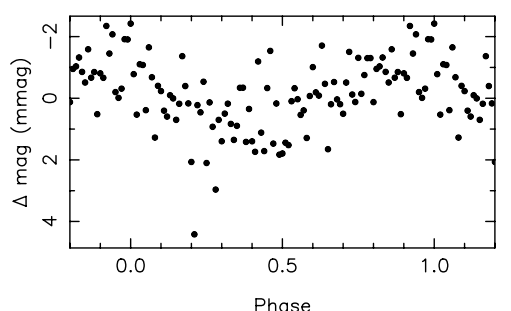

Ren ID 6681 (1SWASPJ041017.54-030818.5)

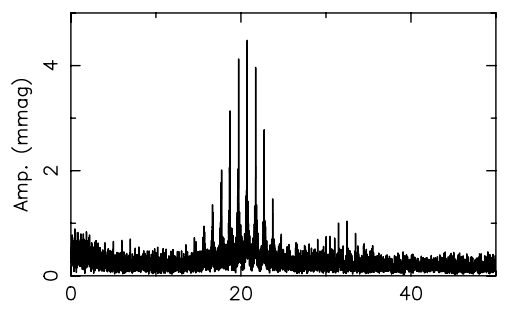

Freq. $\left(d^{-1}\right)$

Frequency $20.730732 d^{-1}$

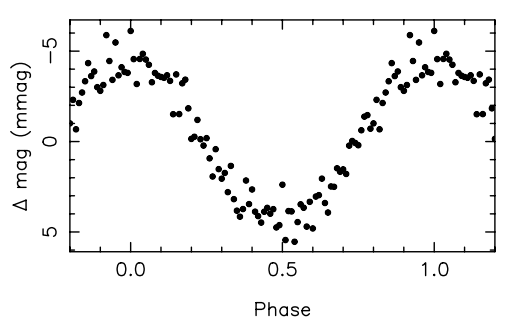

Ren ID 6295 (1SWASPJ035611.62+570512.3)

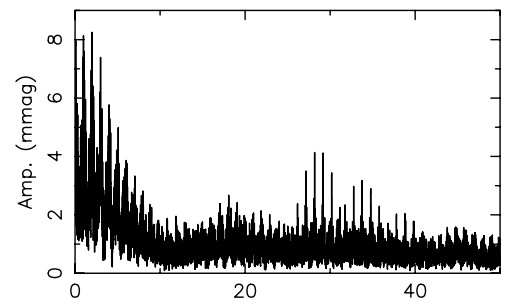

Freq. $\left(d^{-1}\right)$

Frequency $28.1773891 d^{-1}$

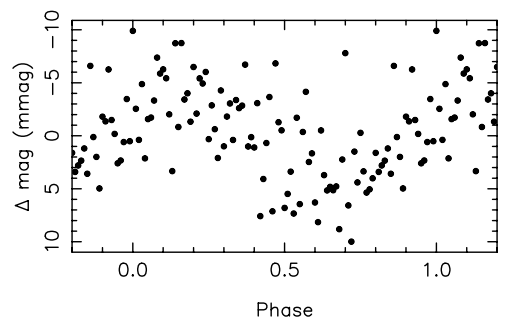

Ren ID 6463 (1SWASPJ040137.56-025433.6)

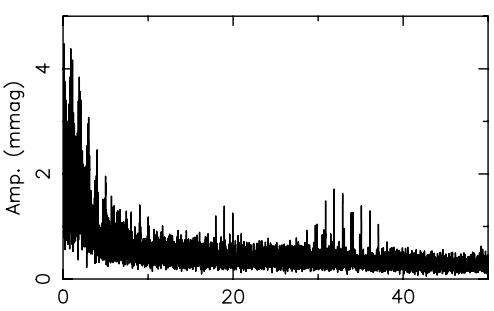

Freq. $\left(d^{-1}\right)$

Frequency $31.8867283 d^{-1}$

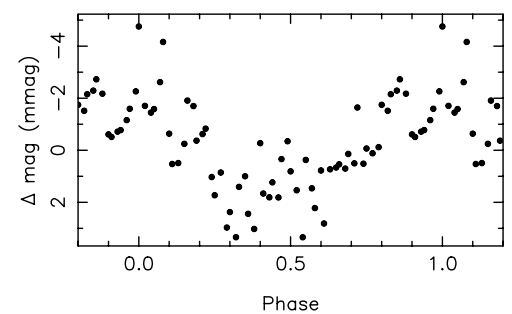

Ren ID 6663 (1SWASPJ041142.67+570754.9)

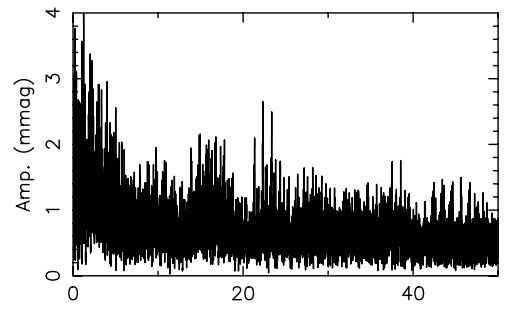

Freq. $\left(d^{-1}\right)$

Frequency $22.3602047 d^{-1}$

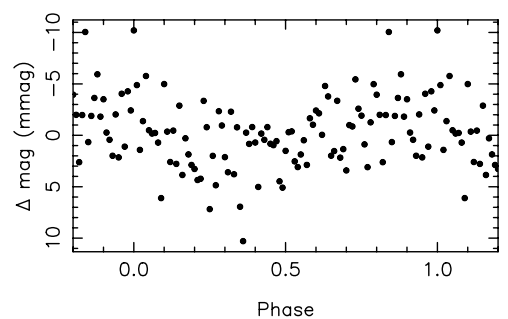

Ren ID 6390 (1SWASPJ035711.24-405420.6)

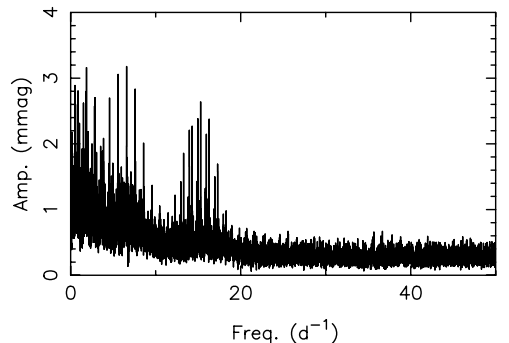

Frequency $6.5758028 \mathrm{~d}^{-1}$

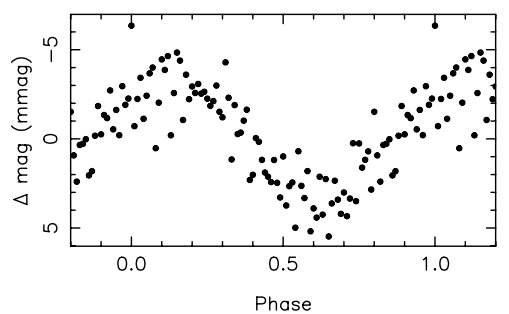

Ren ID 6527 (1SWASPJ040358.68-060306.6)

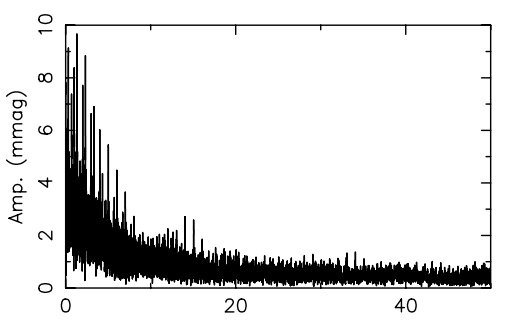

Freq. $\left(d^{-1}\right)$

Frequency $1.3182503 \mathrm{~d}^{-1}$

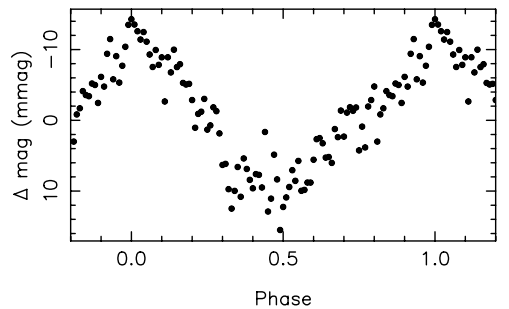

Ren ID 8720 (1SWASPJ051432.77-411443.0)

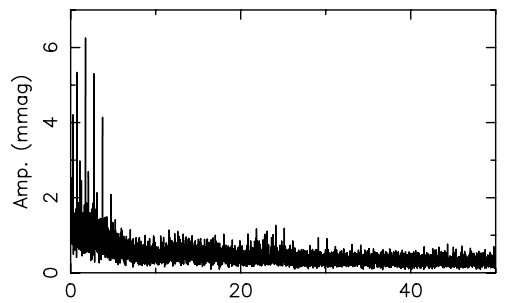

Freq. $\left(d^{-1}\right)$

Frequency $1.74240255 \mathrm{~d}^{-1}$

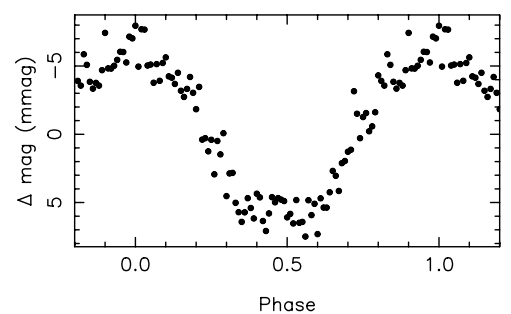

Fig. 1. continued. 
B. Smalley et al.: SuperWASP observations of pulsating Am stars

Ren ID 8711 (1SWASPJ051710.94+331613.2)

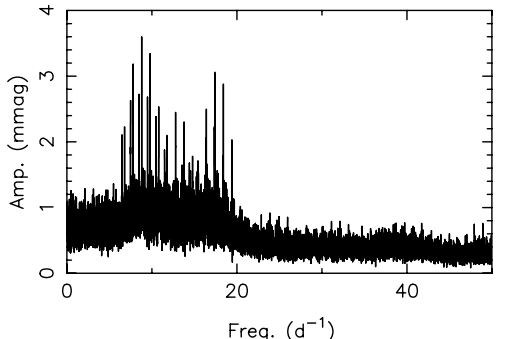

Frequency $8.78979111 \mathrm{~d}^{-1}$

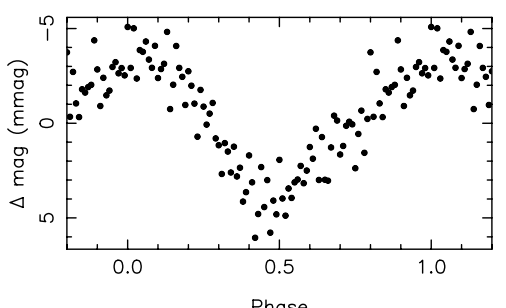

Ren ID 8932 (1SWASPJ052230.29+291548.7)

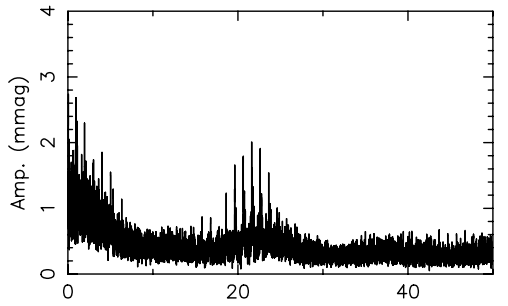

Freq. $\left(d^{-1}\right)$

Frequency $21.6154137 d^{-1}$

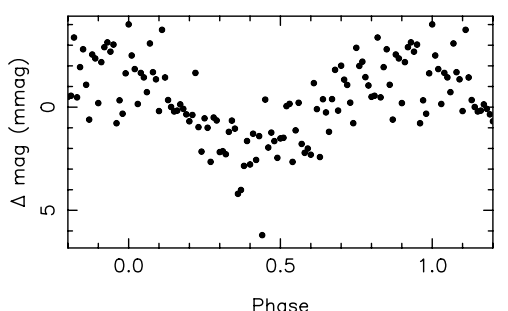

Ren ID 8974 (1SWASPJ052343.42+302219.2)
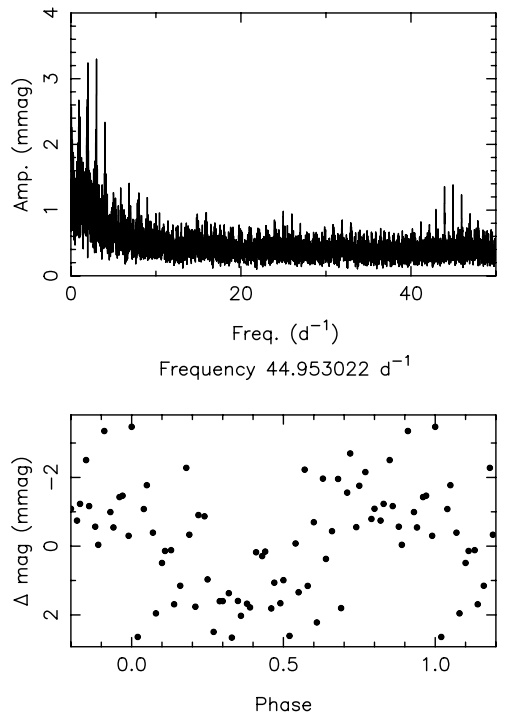

Ren ID 8842 (1SWASPJ052000.44+242526.2)

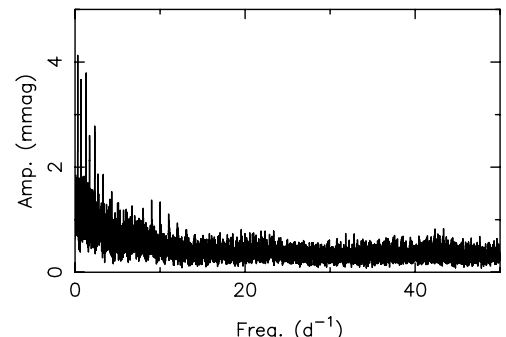

Frequency $0.304347098 d^{-1}$

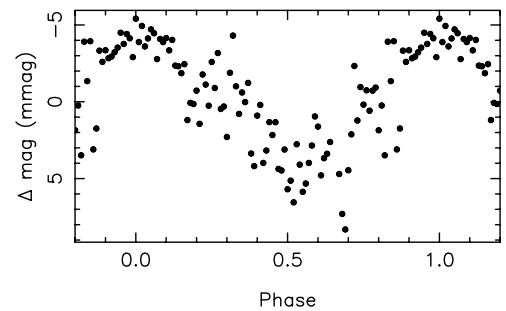

Ren ID 8951 (1SWASPJ052247.99+234332.7)

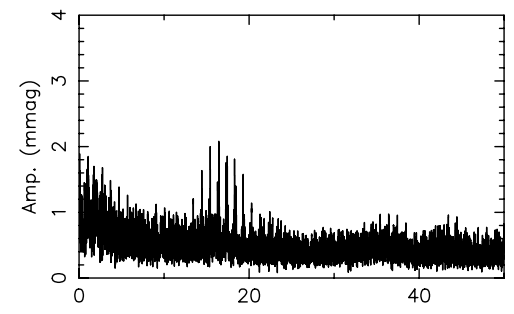

Freq. $\left(d^{-1}\right)$

Frequency $16.4352798 d^{-1}$

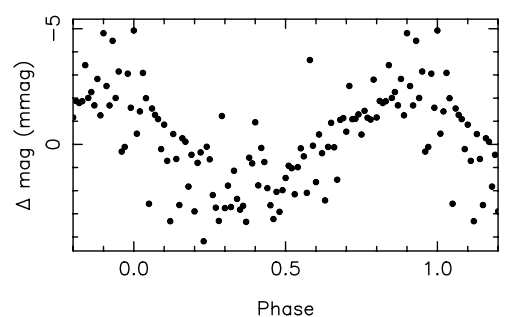

Ren ID 8988 (1SWASPJ052447.95+332047.8)

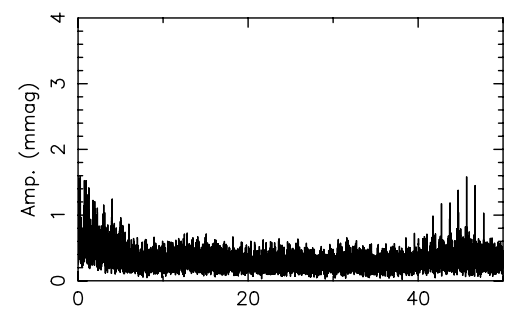

Freq. $\left(d^{-1}\right)$

Frequency $45.7112083 d^{-1}$

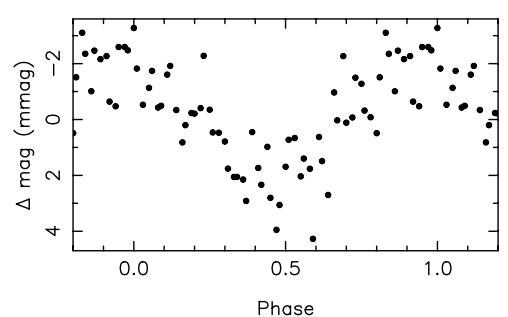

Ren ID 8898 (1SWASPJ052156.12+332311.5)

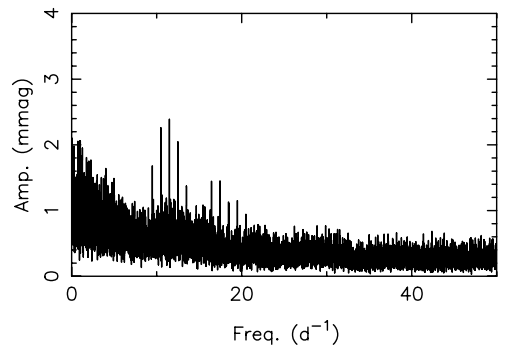

Frequency $11.4942684 d^{-1}$

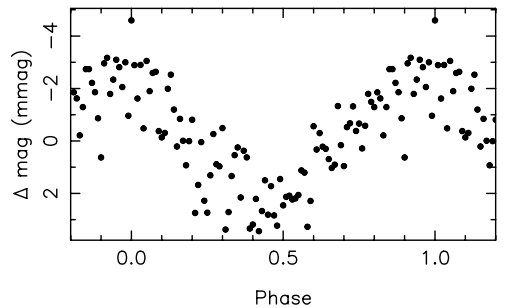

Ren ID 8972 (1SWASPJ052341.88+320649.9)

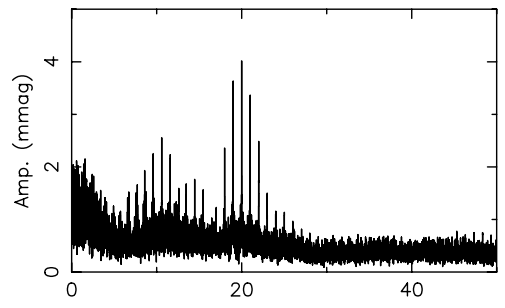

Freq. $\left(d^{-1}\right)$

Frequency $19.9968987 d^{-1}$

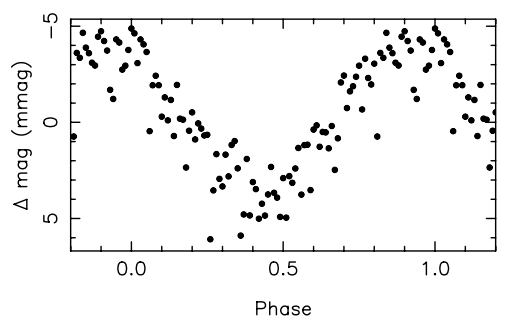

Ren ID 9084 (1SWASPJ052618.78+335304.6)

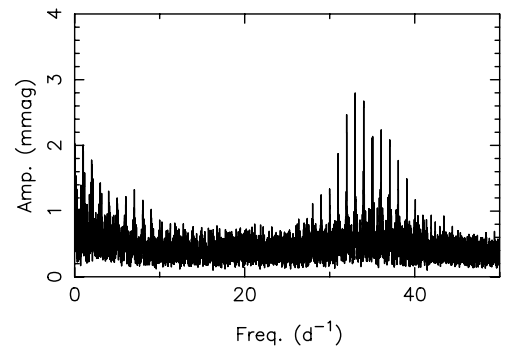

Frequency $33.0007286 d^{-1}$

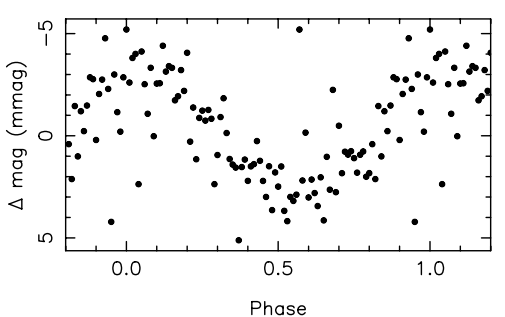

Fig. 1. continued. 
Ren ID 9123 (1SWASPJ052626.08+303712.6)

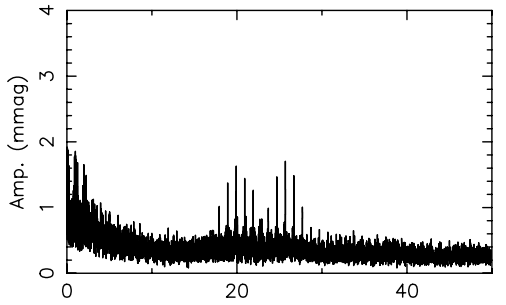

Freq. $\left(d^{-1}\right)$

Frequency $25.6982899 d^{-1}$

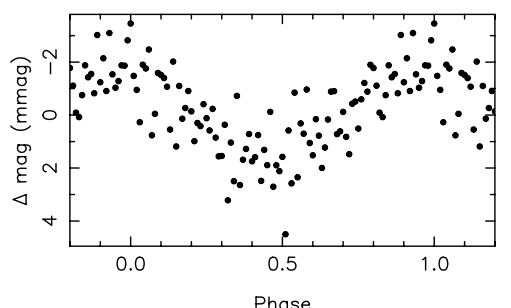

Ren ID 9269 (1SWASPJ052952.61+294436.9)

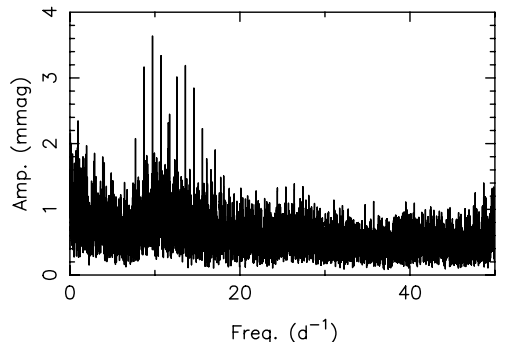

Frequency $9.71830368 d^{-1}$

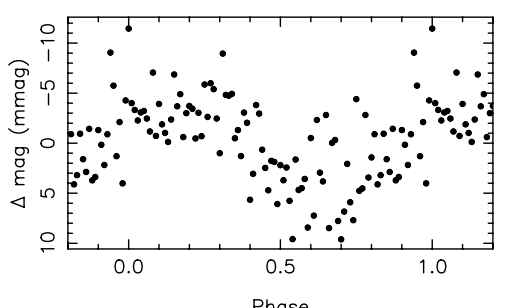

Ren ID 9454 (1SWASPJ053311.31+240705.3)

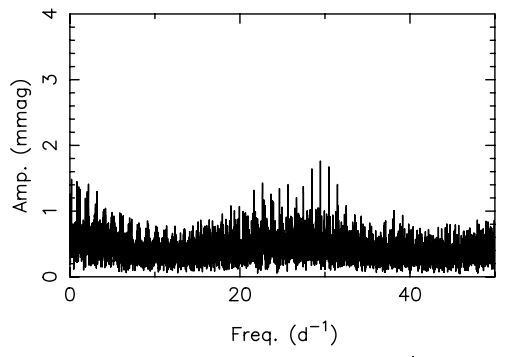

Frequency $29.4675198 d^{-1}$

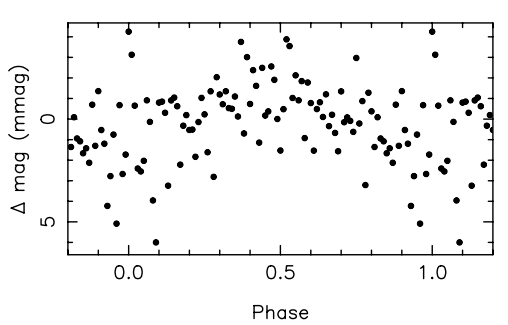

Ren ID 9144 (1SWASPJ052653.92+323251.0)

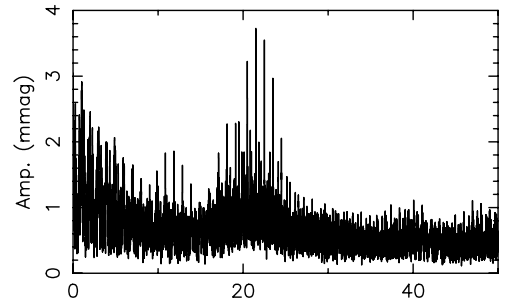

Freq. $\left(d^{-1}\right)$

Frequency $21.4997215 d^{-1}$

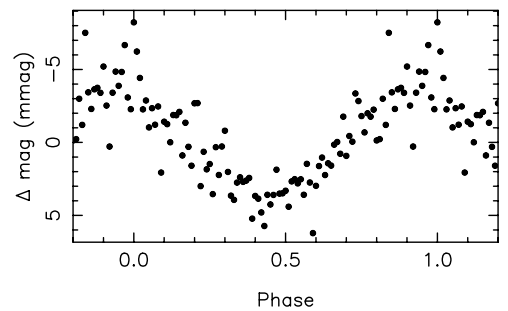

Ren ID 9470 (1SWASPJ053201.99-071524.8)

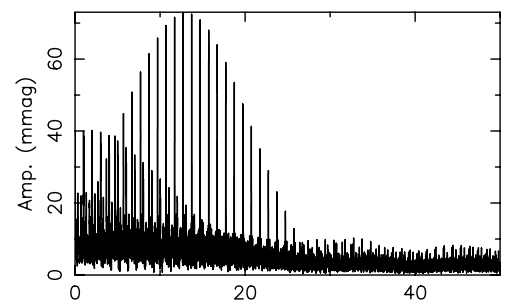

Freq. $\left(d^{-1}\right)$

Frequency $12.7087898 d^{-1}$

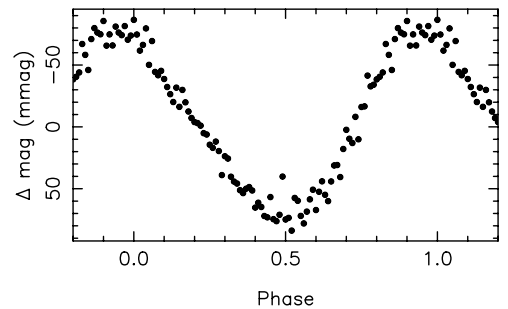

Ren ID 9685 (1SWASPJ053353.83-224406.3)

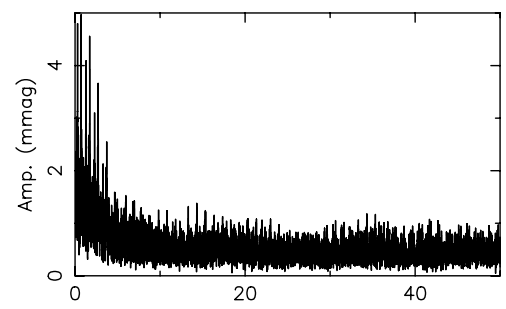

Freq. $\left(d^{-1}\right)$

Frequency $0.711294234 d^{-1}$

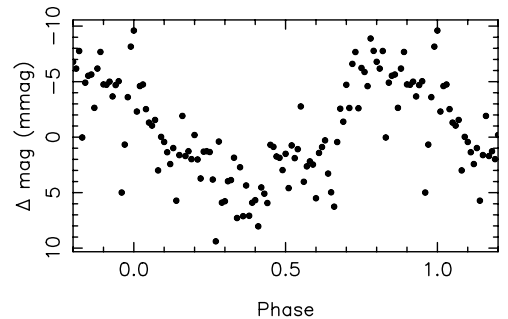

Ren ID 9262 (1SWASPJ052927.84+323027.8)

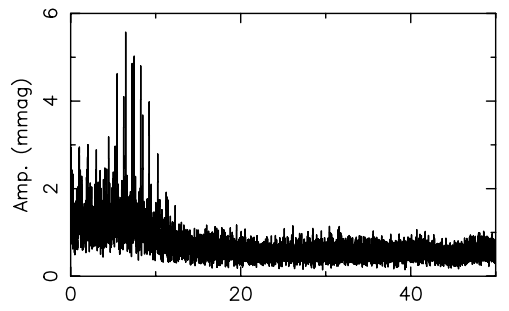

Freq. $\left(d^{-1}\right)$

Frequency $6.46808863 d^{-1}$

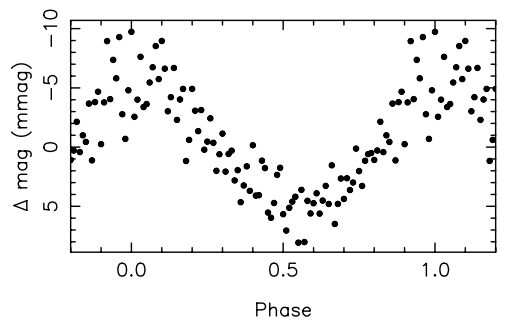

Ren ID 9375 (1SWASPJ053232.84+345030.8)

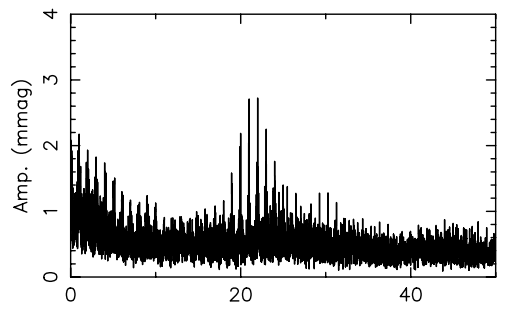

Freq. $\left(d^{-1}\right)$

Frequency $21.9980488 d^{-1}$

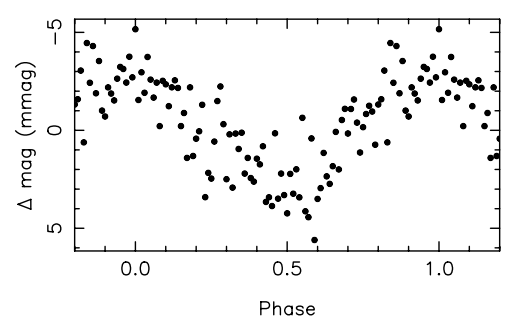

Ren ID 9534 (1SWASPJ053417.42+313123.9)

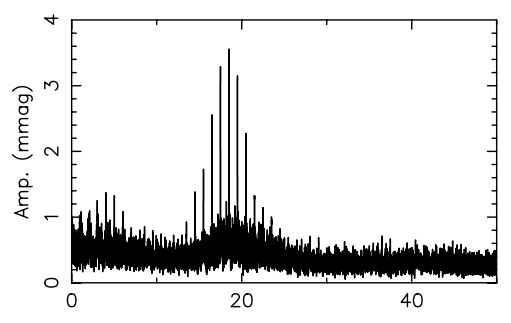

Freq. $\left(d^{-1}\right)$

Frequency $18.5025463 d^{-1}$

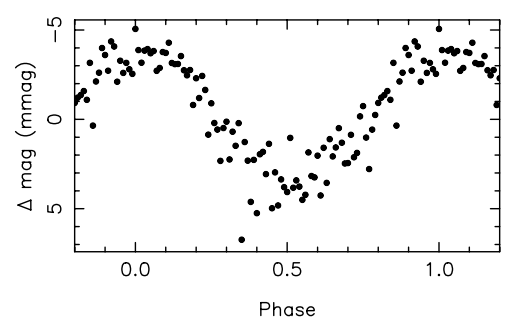

Fig. 1. continued. 
B. Smalley et al.: SuperWASP observations of pulsating Am stars

Ren ID 9581 (1SWASPJ053452.74+313808.5)

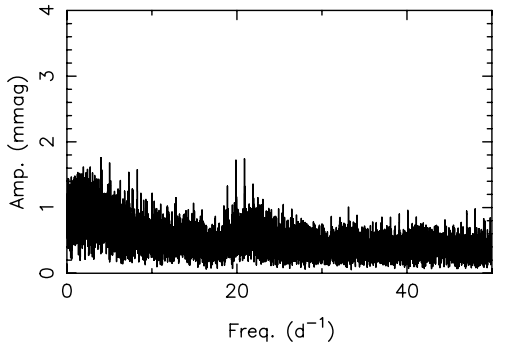

Frequency $20.8886681 d^{-1}$

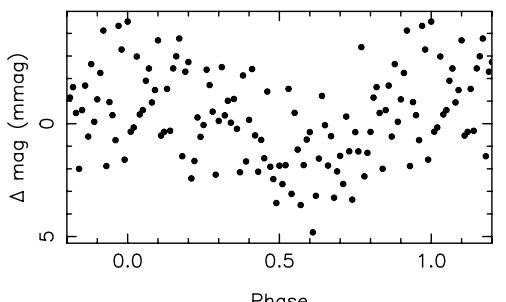

Ren ID 9812 (1SWASPJ053646.85+331254.1)

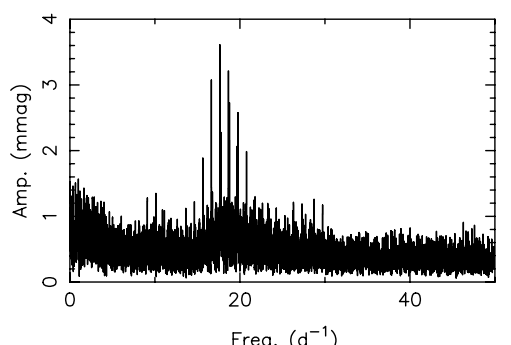

Frequency $17.6418152 d^{-1}$

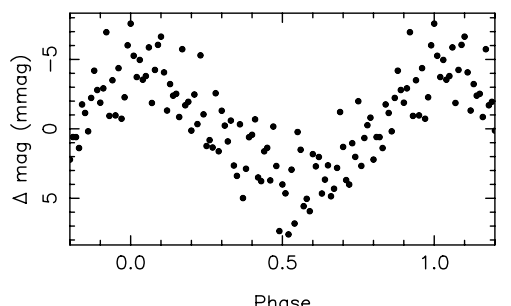

Ren ID 10259 (1SWASPJ054518.88+335223.9)

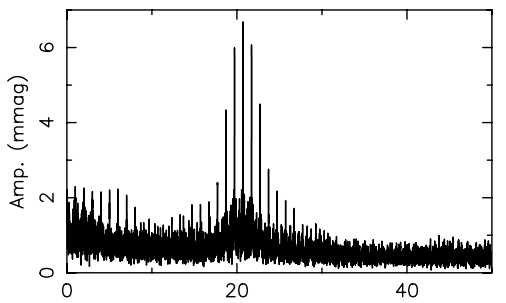

Freq. $\left(d^{-1}\right)$

Frequency $20.7162228 d^{-1}$

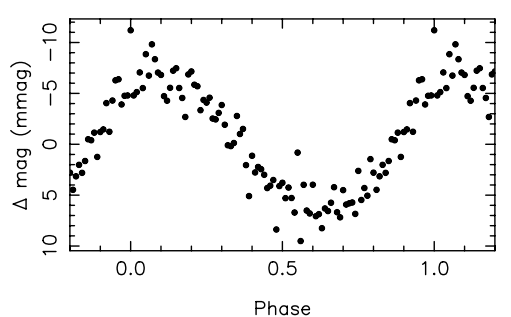

Ren ID 9556 (1SWASPJ053455.16+343849.3)

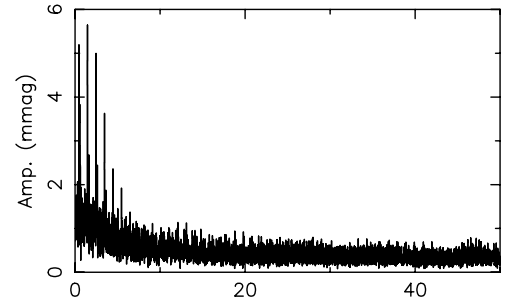

Freq. $\left(d^{-1}\right)$

Frequency $1.45707655 d^{-1}$

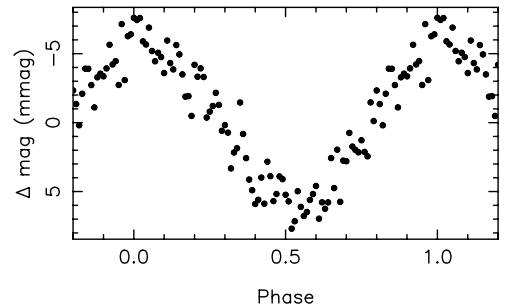

Ren ID 9868 (1SWASPJ053730.35+322621.6)

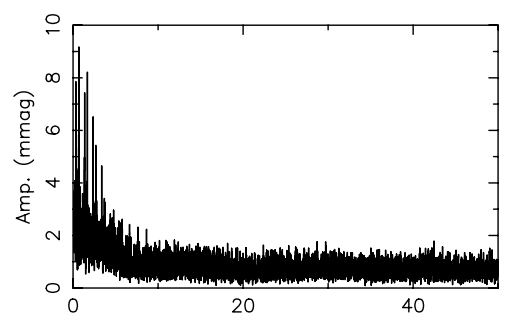

Freq. $\left(d^{-1}\right)$

Frequency $0.678587794 d^{-1}$

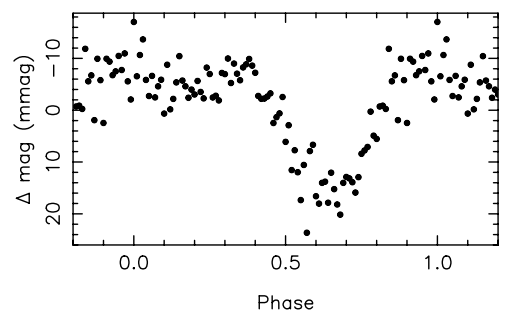

Ren ID 10383 (1SWASPJ054812.18+294604.7)

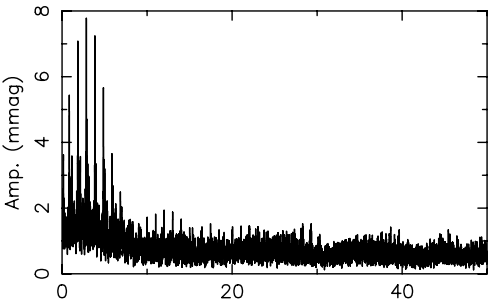

Freq. $\left(d^{-1}\right)$

Frequency $2.85380125 d^{-1}$

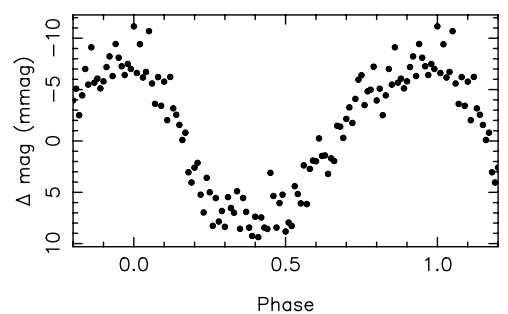

Ren ID 9653 (1SWASPJ053547.14+323232.6)

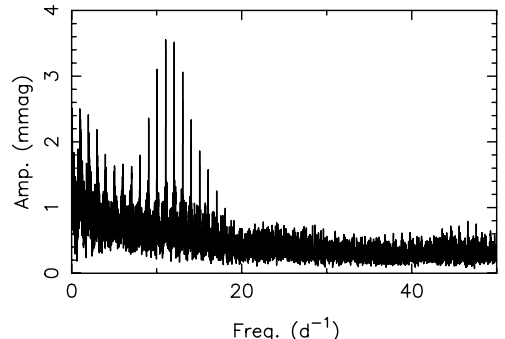

Frequency $11.0582571 \mathrm{~d}^{-1}$

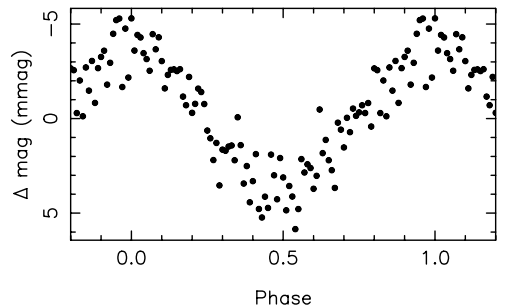

Ren ID 10206 (1SWASPJ054227.04+270237.4)

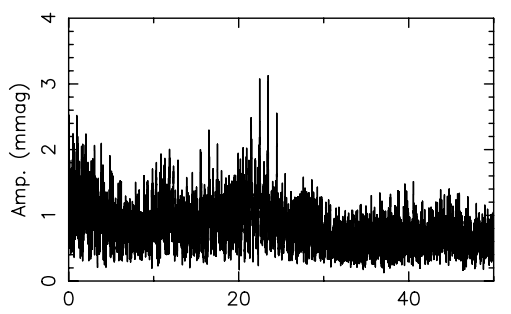

Freq. $\left(d^{-1}\right)$

Frequency $23.4739361 d^{-1}$

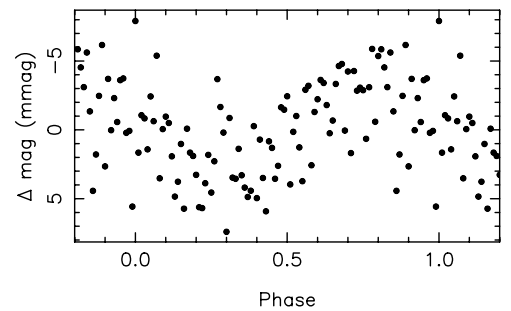

Ren ID 10423 (1SWASPJ054904.81+261521.5)

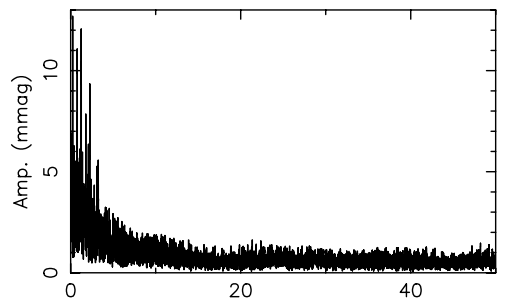

Freq. $\left(d^{-1}\right)$

Frequency $0.233571857 d^{-1}$

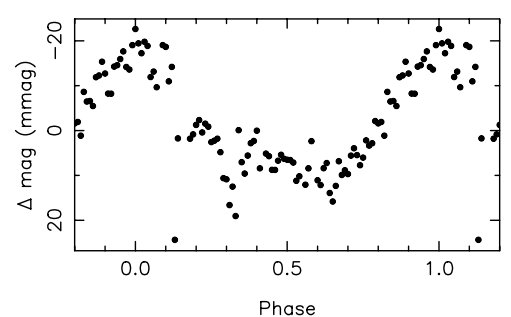

Fig. 1. continued. 


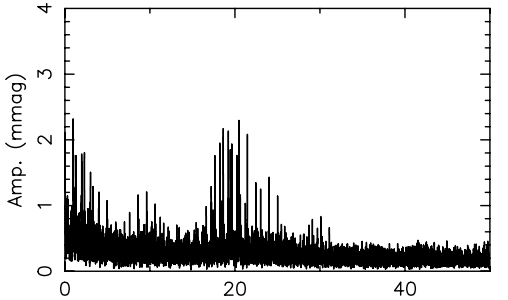

Freq. $\left(d^{-1}\right)$

Frequency $20.46278 d^{-1}$

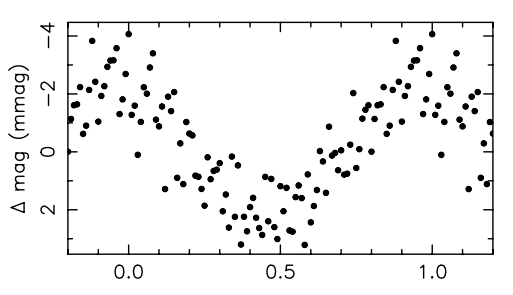

Phase

Ren ID 10482 (1SWASPJ055122.90+335136.0)

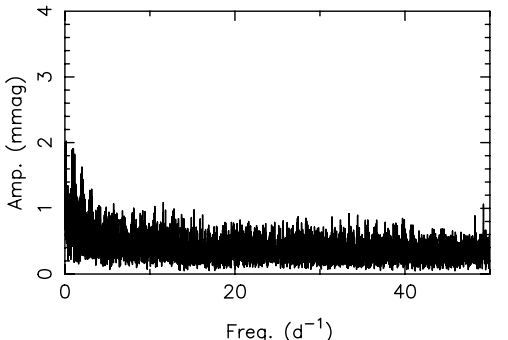

Frequency $49.2363129 \mathrm{~d}^{-1}$

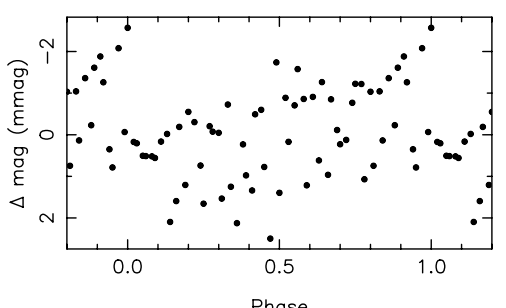

Ren ID 10586 (1SWASPJ055318.29+244704.3)

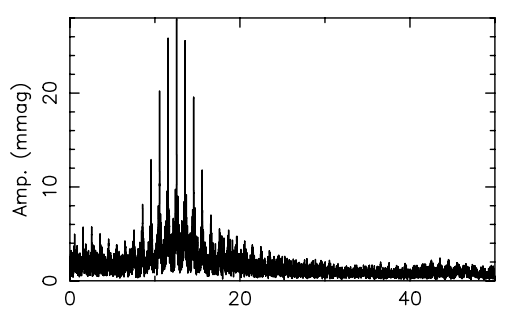

Freq. $\left(d^{-1}\right)$

Frequency $12.549674 d^{-1}$

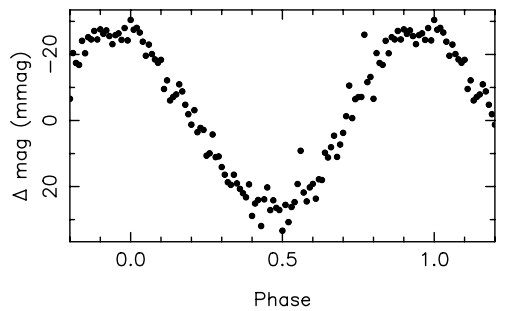

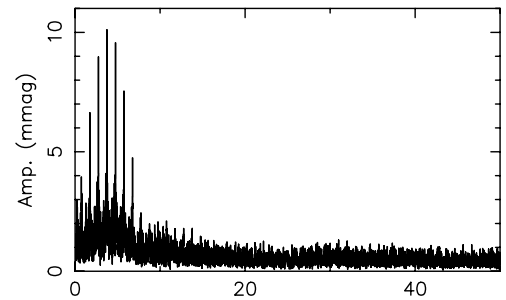

Freq. $\left(d^{-1}\right)$

Frequency $3.74725747 d^{-1}$

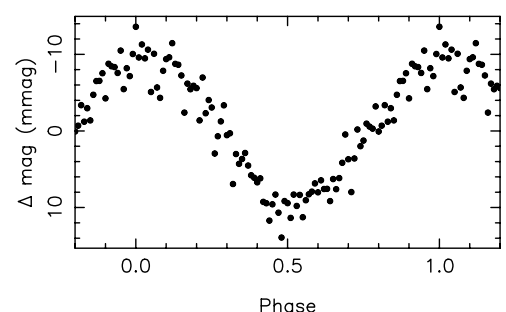

Ren ID 10565 (1SWASPJ055254.70+245759.6)

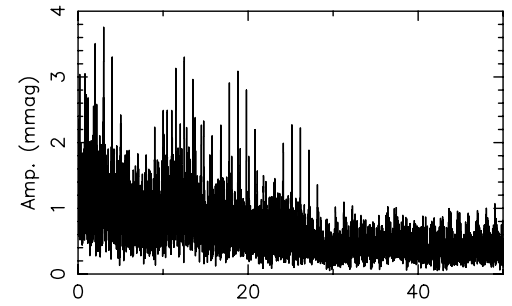

Freq. $\left(d^{-1}\right)$

Frequency $12.4952059 d^{-1}$

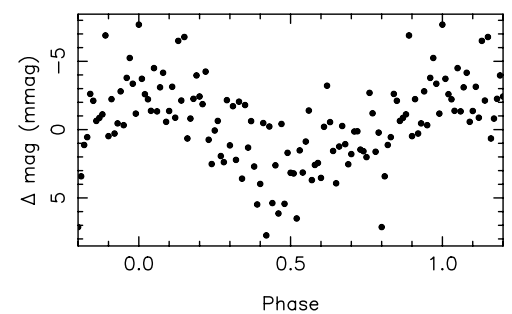

Ren ID 10602 (1SWASPJ055422.75+305401.8)

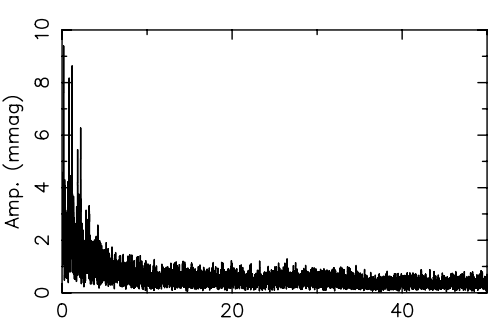

Freq. $\left(d^{-1}\right)$

Frequency $0.177097946 d^{-1}$

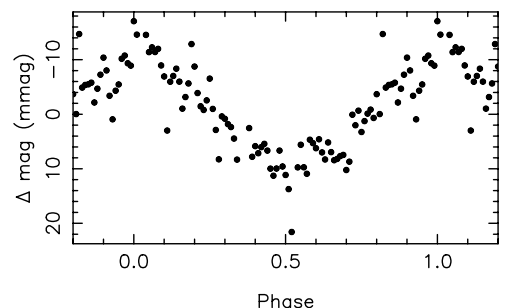

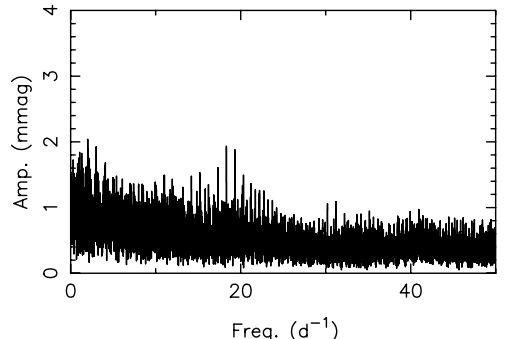

Frequency $18.3207073 d^{-1}$

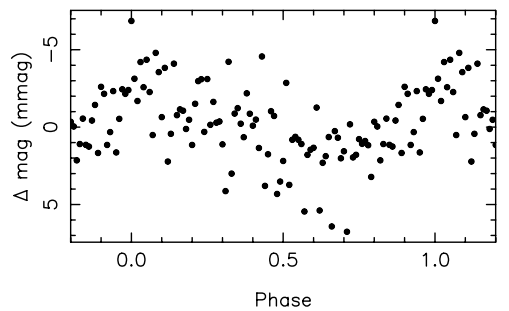

Ren ID 10554 (1SWASPJ055256.62+301210.5)

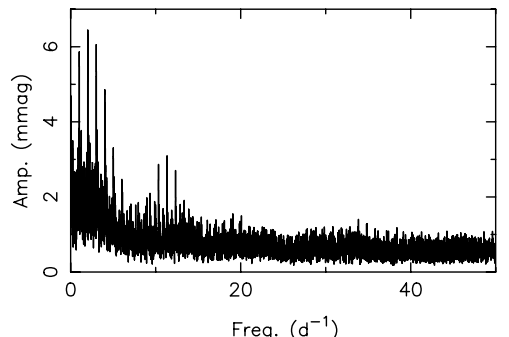

Frequency $11.3344879 d^{-1}$

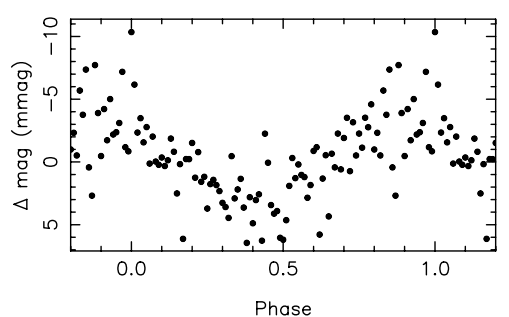

Ren ID 10641 (1SWASPJ055628.80+295932.3)

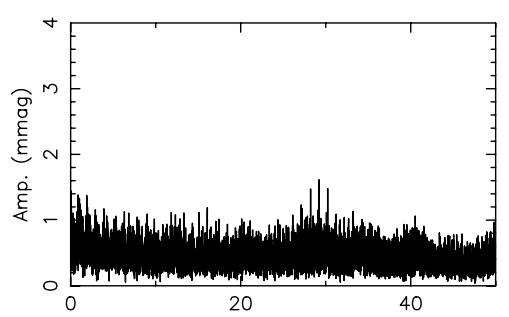

Freq. $\left(d^{-1}\right)$

Frequency $29.2306995 d^{-1}$

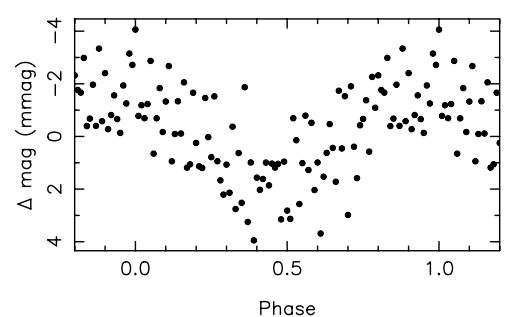

Fig. 1. continued. 
B. Smalley et al.: SuperWASP observations of pulsating Am stars

Ren ID 10855 (1SWASPJ060105.58+284706.4)

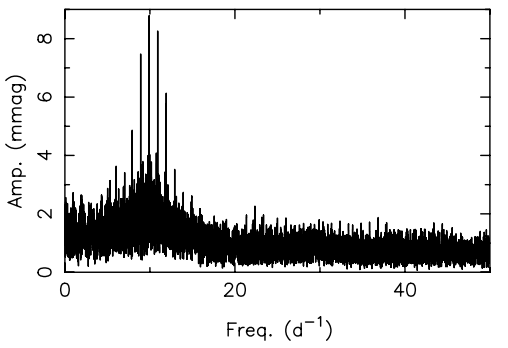

Frequency $9.91084671 \mathrm{~d}^{-1}$

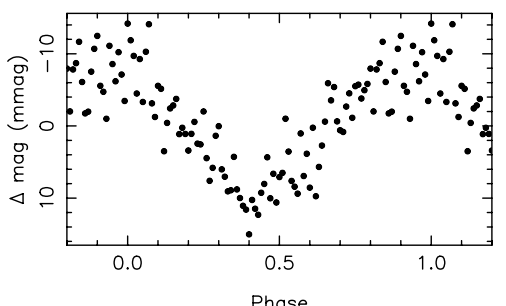

Ren ID 11025 (1SWASPJ060509.01+293459.5)

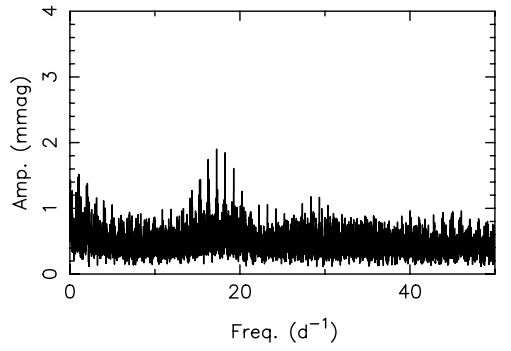

Frequency $17.256258 d^{-1}$

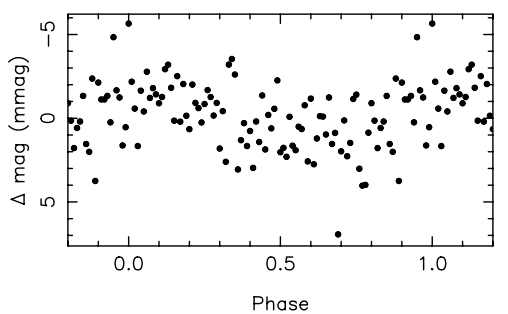

Ren ID 11135 (1SWASPJ060825.42+331752.4)

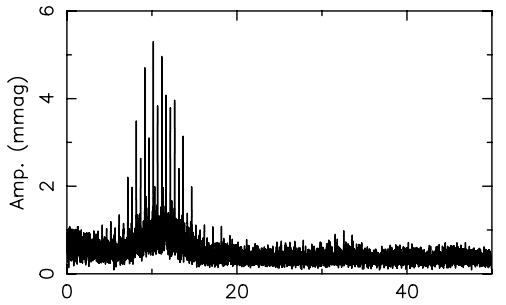

Freq. $\left(d^{-1}\right)$

Frequency $10.1654596 d^{-1}$

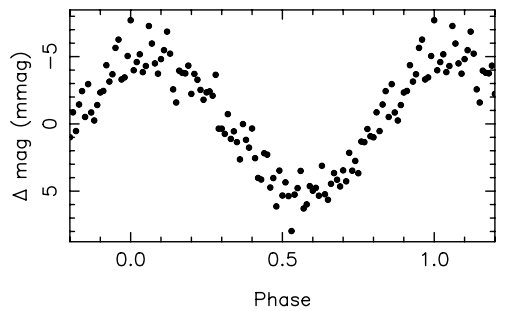

Ren ID 11007 (1SWASPJ060432.59+245925.7)

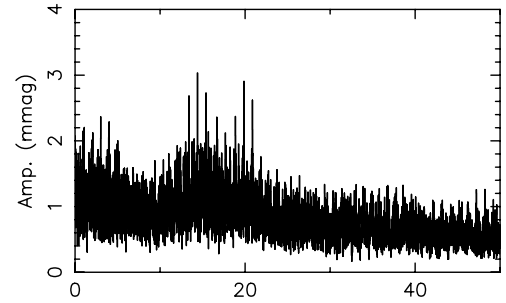

Freq. $\left(d^{-1}\right)$

Frequency $14.3992662 d^{-1}$

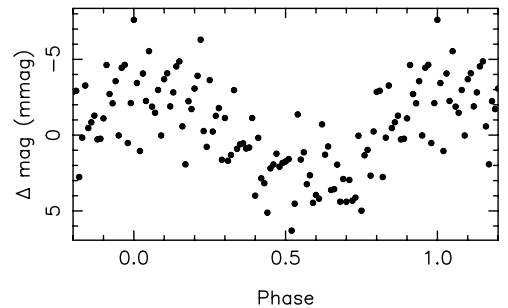

Ren ID 11032 (1SWASPJ060526.26+290149.0)

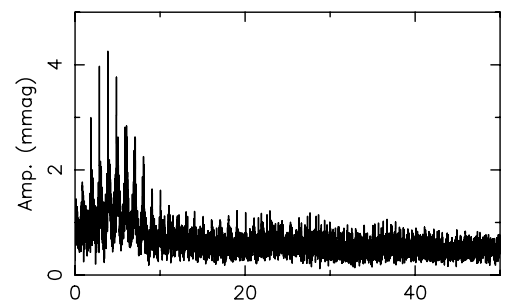

Freq. $\left(d^{-1}\right)$

Frequency $3.85902905 d^{-1}$

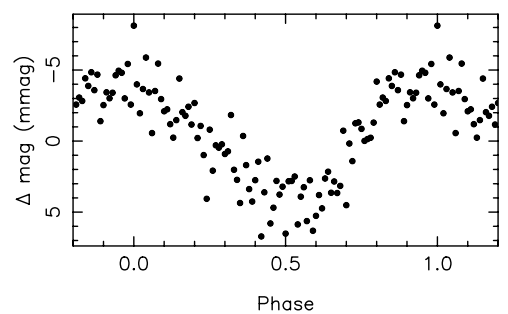

Ren ID 11184 (1SWASPJ060853.32+234828.0)

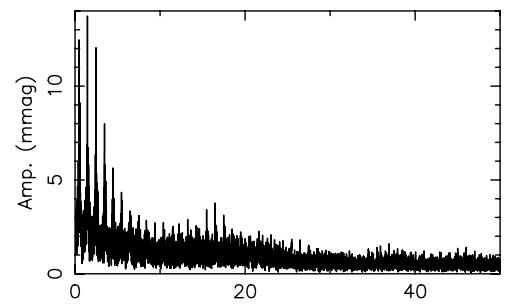

Freq. $\left(d^{-1}\right)$

Frequency $1.45250511 \mathrm{~d}^{-1}$

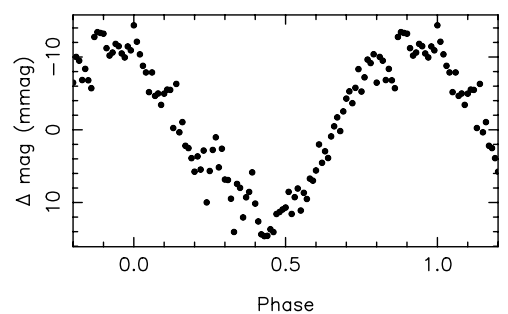

Ren ID 11022 (1SWASPJ060503.38+305522.5)

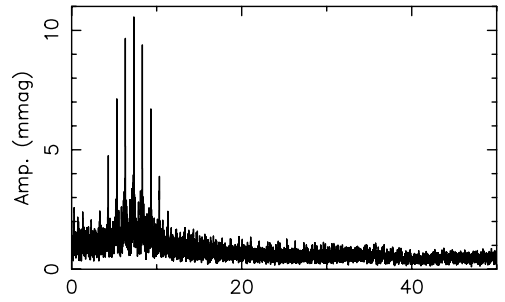

Freq. $\left(d^{-1}\right)$

Frequency $7.31085253 d^{-1}$

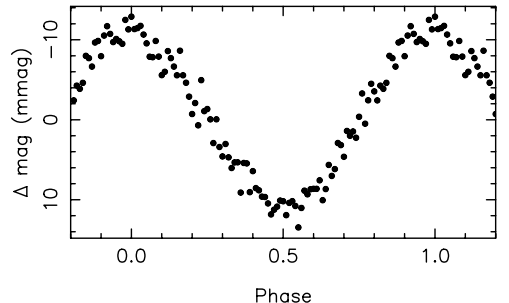

Ren ID 11033 (1SWASPJ060536.05+315120.1)

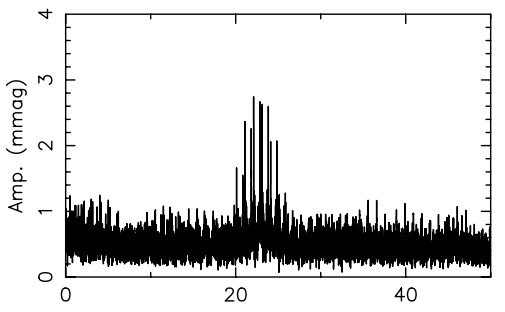

Freq. $\left(d^{-1}\right)$

Frequency $22.1083775 d^{-1}$

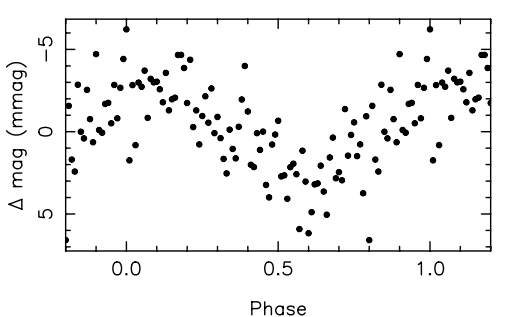

Ren ID 11182 (1SWASPJ060903.53+294515.0)

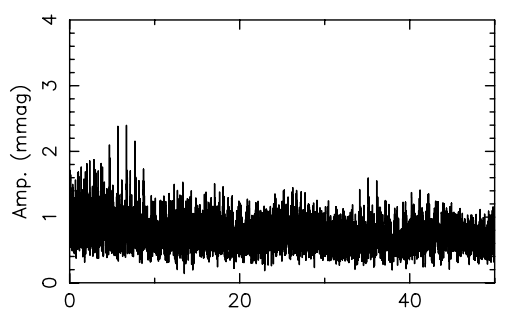

Freq. $\left(d^{-1}\right)$

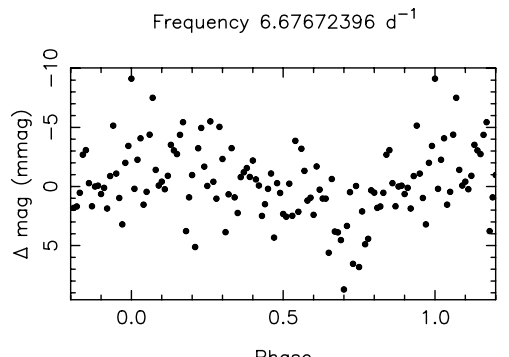

Fig. 1. continued. 

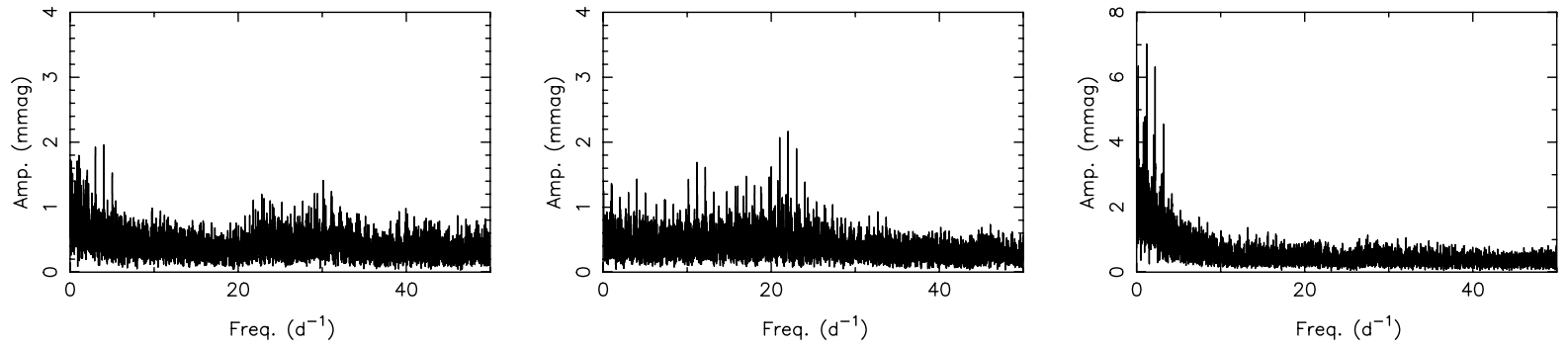

Frequency $30.1049366 d^{-1}$

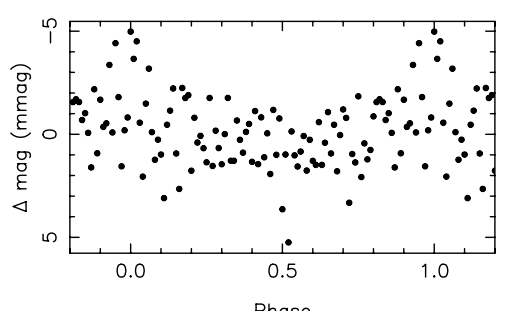

Frequency $22.0072556 d^{-1}$

Frequency $1.19433522 d^{-1}$
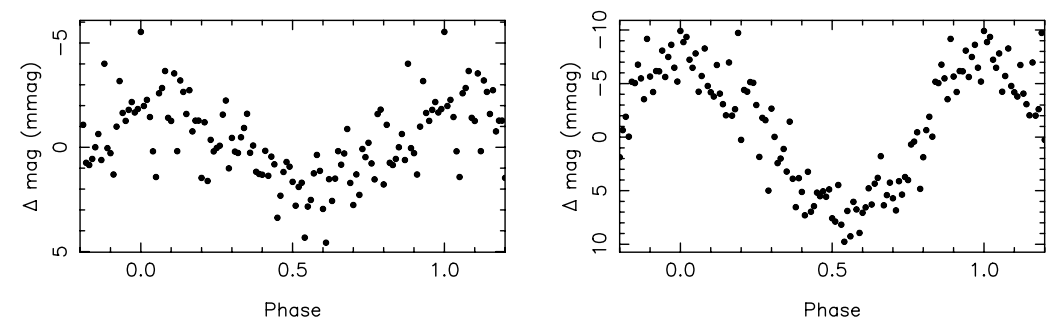

Ren ID 11820 (1SWASPJ061920.63-545156.3)

Ren ID 12250 (1SWASPJ062745.34-450154.6)

Ren ID 12820 (1SWASPJ063857.69-270712.3)
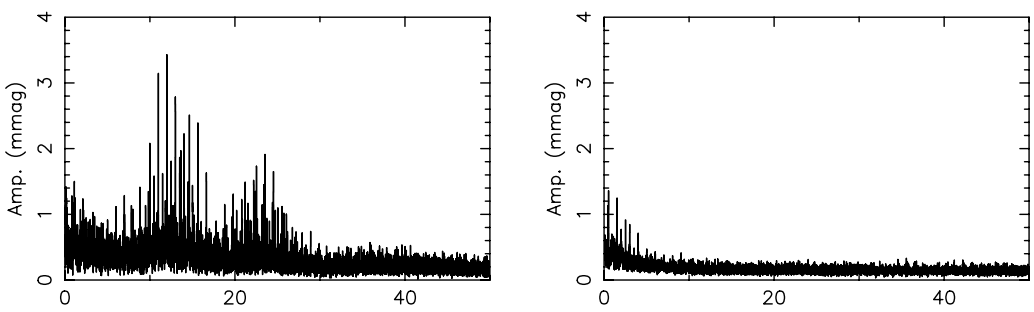

Freq. $\left(d^{-1}\right)$

Frequency $11.9914322 d^{-1}$

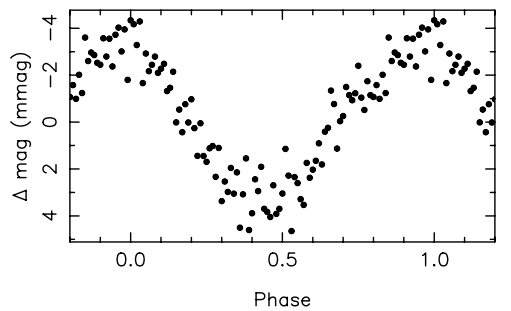

Freq. $\left(d^{-1}\right)$

Frequency $0.520913184 d^{-1}$

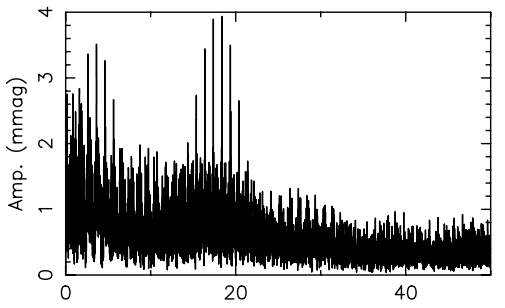

Freq. $\left(d^{-1}\right)$

Frequency $18.3705025 d^{-1}$
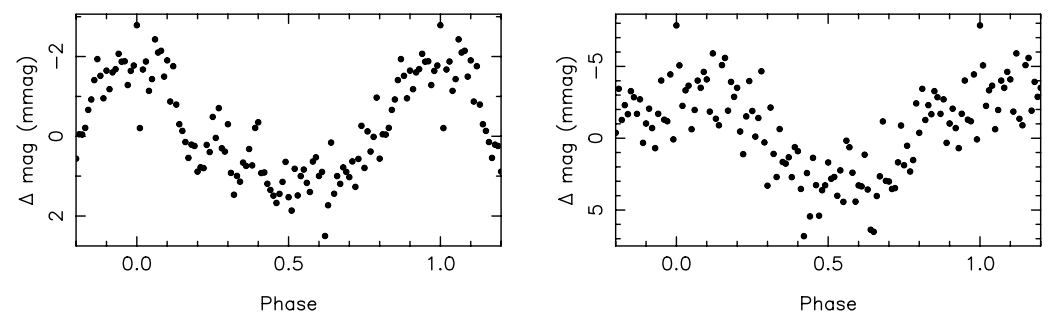

Ren ID 13550 (1SWASPJ064704.03-604331.6)

Ren ID 14140 (1SWASPJ065418.80-490217.1)

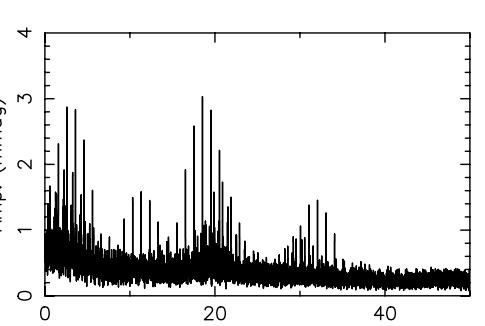

Freq. $\left(d^{-1}\right)$

Frequency $18.5330791 \mathrm{~d}^{-1}$

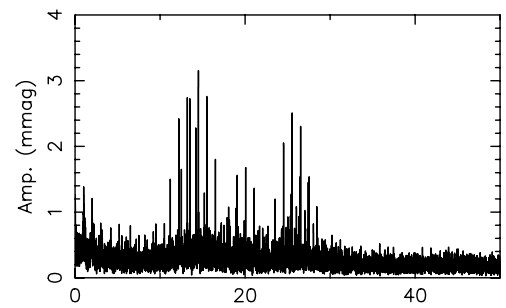

Freq. $\left(d^{-1}\right)$

Frequency $14.5059929 d^{-1}$
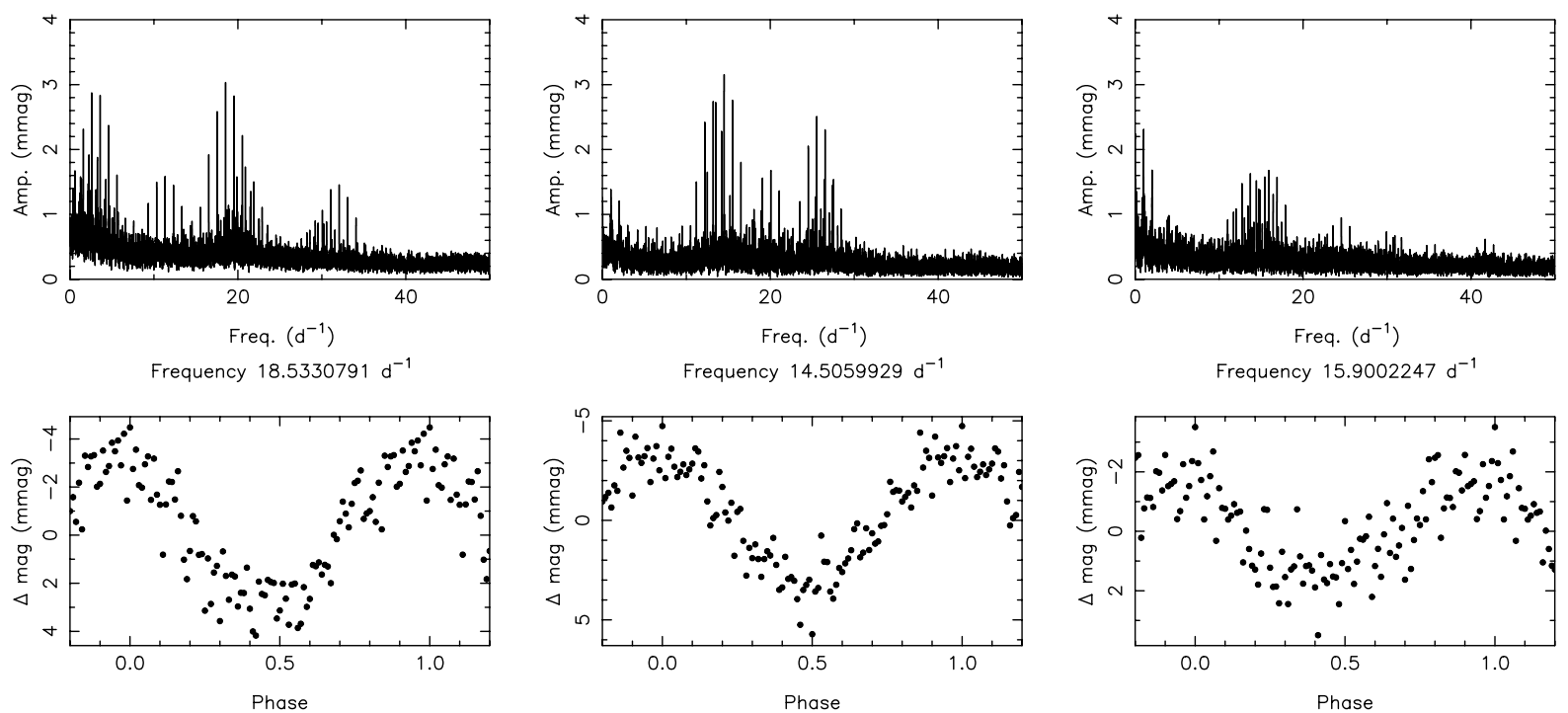

Freq. $\left(d^{-1}\right)$

Frequency $15.9002247 d^{-1}$

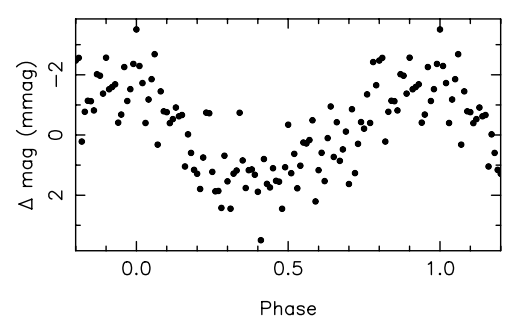

Fig. 1. continued. 
B. Smalley et al.: SuperWASP observations of pulsating Am stars

Ren ID 15423 (1SWASPJ071752.72+193249.6)

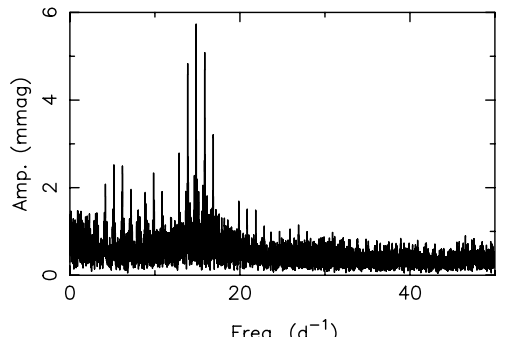

Frequency $14.8532677 d^{-1}$

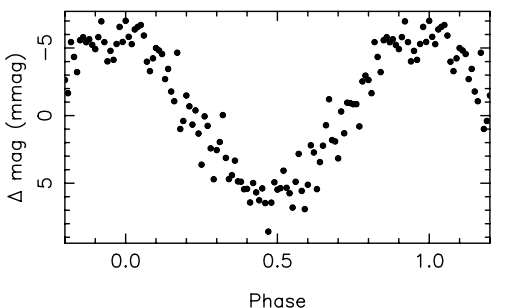

Ren ID 18730 (1SWASPJ081259.05+553730.9)

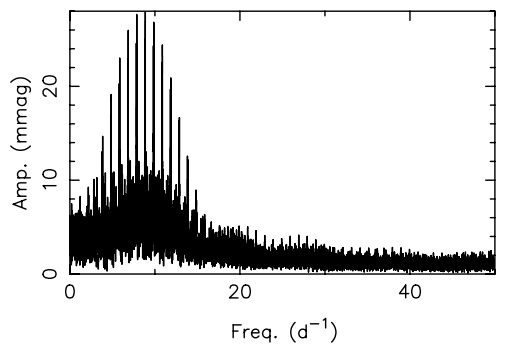

Frequency $8.86711502 d^{-1}$

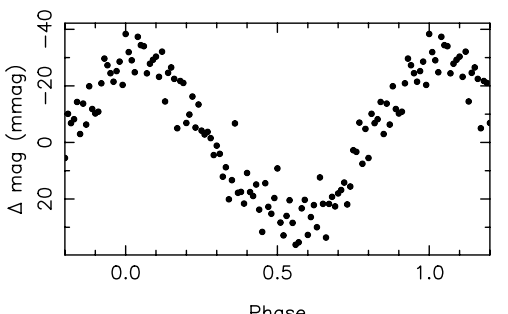

Ren ID 20485 (1SWASPJ083856.27-184234.7)

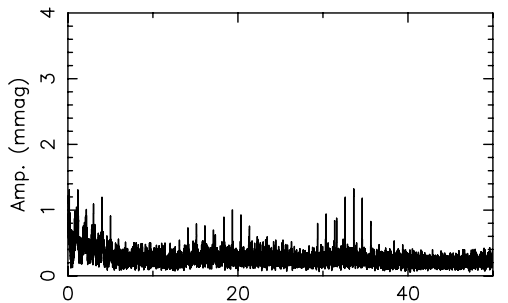

Freq. $\left(d^{-1}\right)$

Frequency $33.623642 d^{-1}$

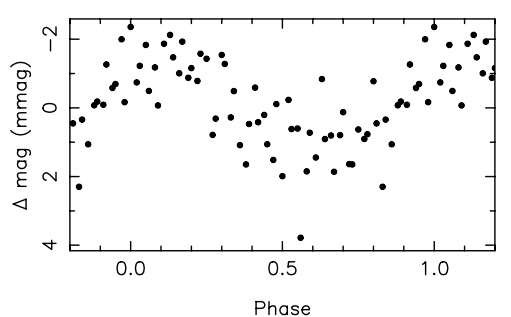

Ren ID 16920 (1SWASPJ074236.24+462928.4)

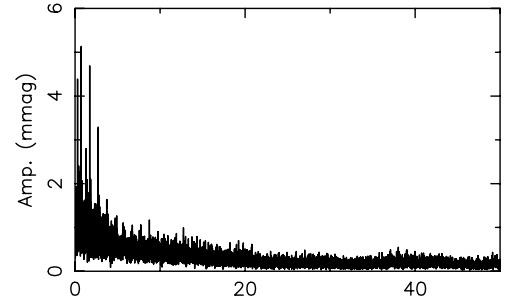

Freq. $\left(d^{-1}\right)$

Frequency $0.71362251 d^{-1}$

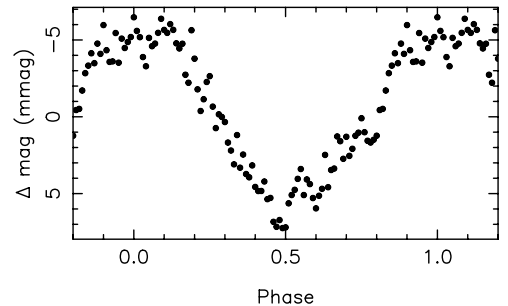

Ren ID 20143 (1SWASPJ083408.68-003156.5)

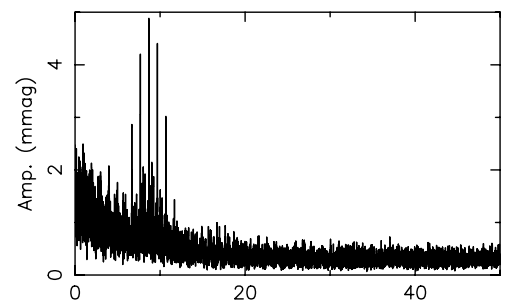

Freq. $\left(d^{-1}\right)$

Frequency $8.67754936 d^{-1}$

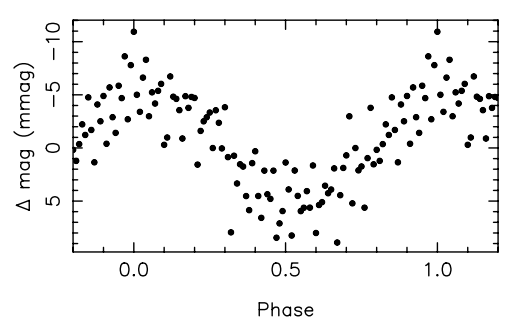

Ren ID 20855 (1SWASPJ084453.32-050446.9)

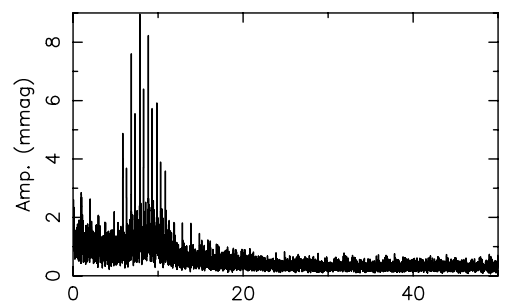

Freq. $\left(d^{-1}\right)$

Frequency $7.84860516 d^{-1}$

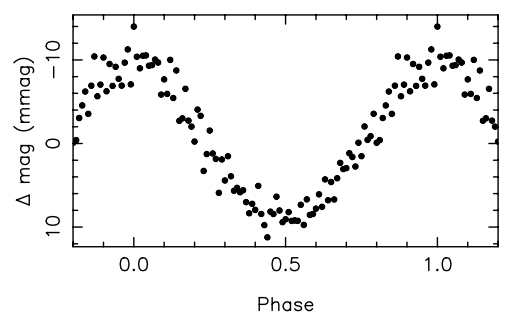

Ren ID 18657 (1SWASPJ080830.19+000755.8)

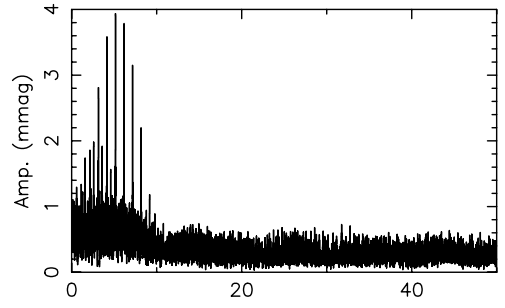

Freq. $\left(d^{-1}\right)$

Frequency $5.15440178 d^{-1}$

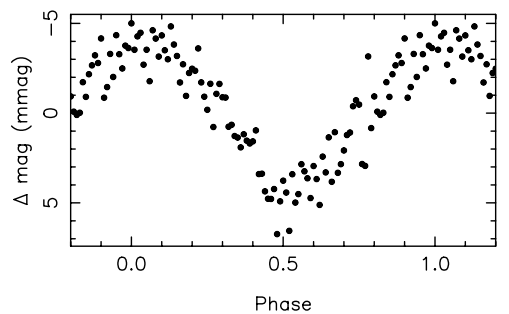

Ren ID 20333 (1SWASPJ083627.01-102709.5)

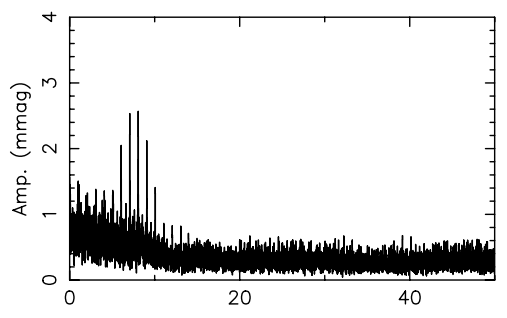

Freq. $\left(d^{-1}\right)$

Frequency $8.06291676 d^{-1}$

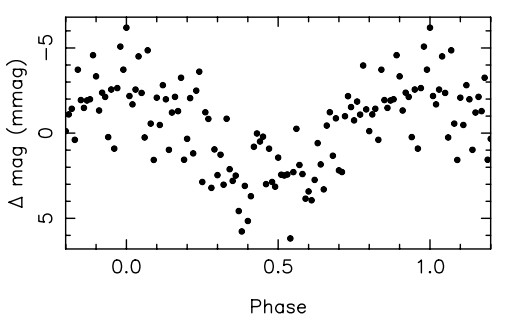

Ren ID 20908 (1SWASPJ084551.93-074252.6)

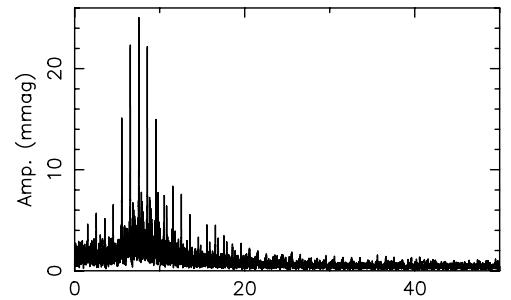

Freq. $\left(d^{-1}\right)$

Frequency $7.54267979 d^{-1}$

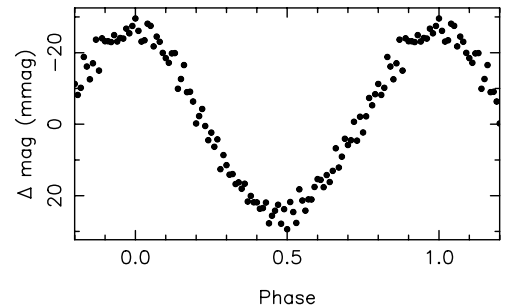

Fig. 1. continued. 

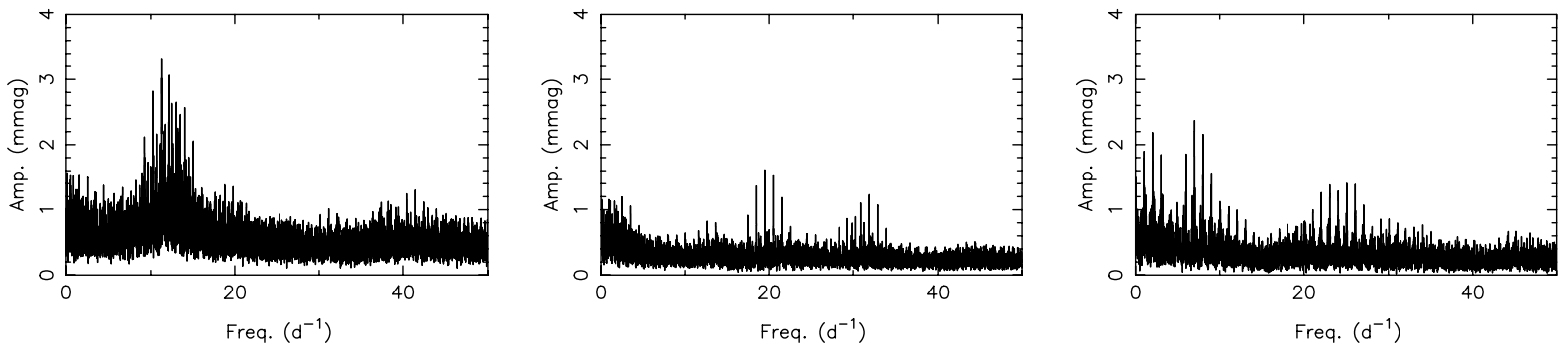

Frequency $11.2578754 d^{-1}$

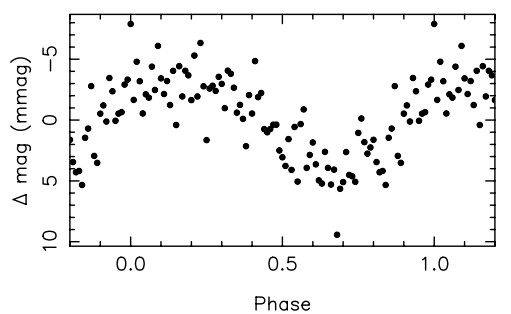

Frequency $19.5074158 d^{-1}$

Frequency $7.01719666 d^{-1}$
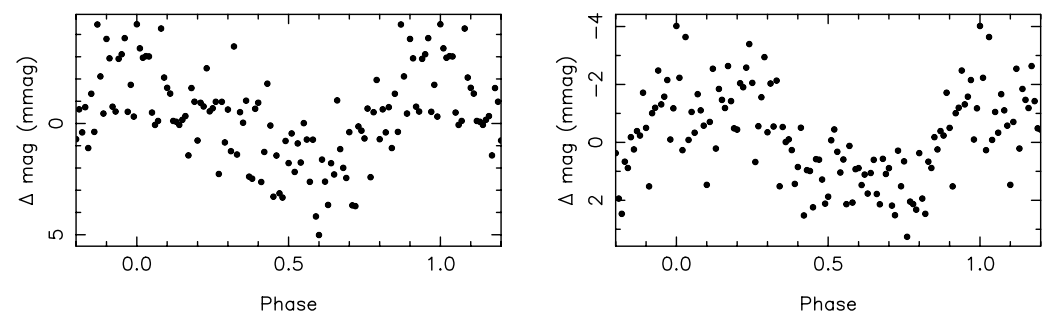

Ren ID 22404 (1SWASPJ091100.59-192859.1)

Ren ID 22450 (1SWASPJ091104.84-344329.0)

Ren ID 22685 (1SWASPJ091518.79-243141.7)
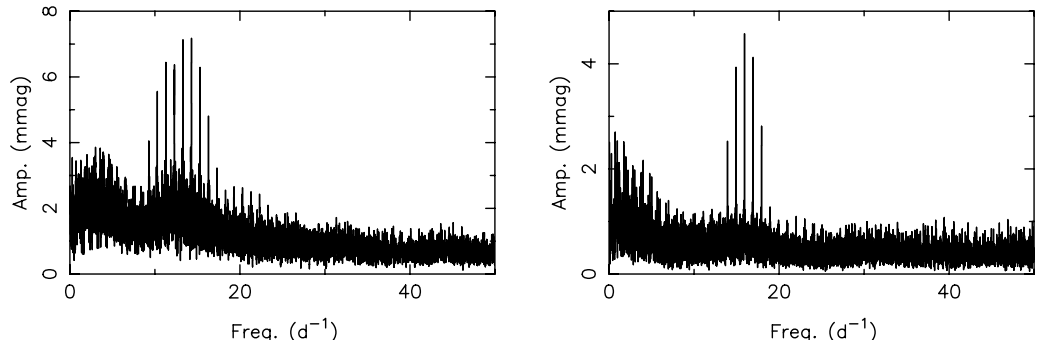

Freq. $\left(d^{-1}\right)$

Frequency $15.9319687 d^{-1}$

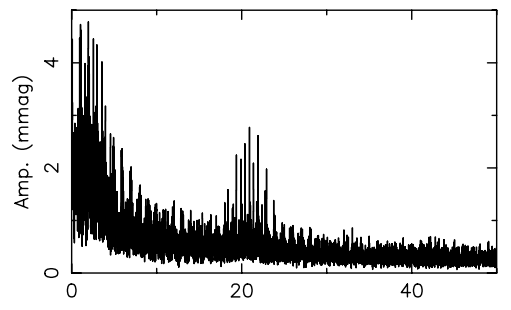

Freq. $\left(d^{-1}\right)$

Frequency $20.9176292 d^{-1}$
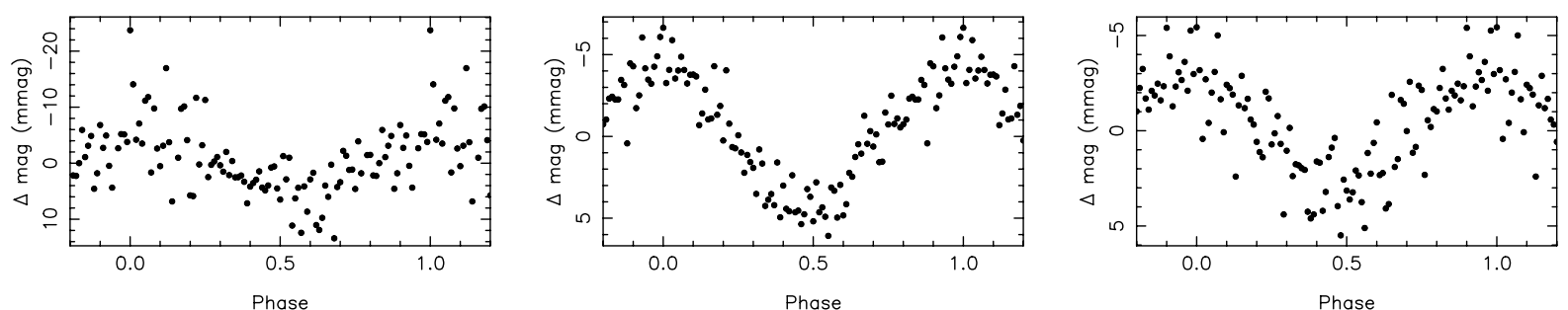

Ren ID 23195 (1SWASPJ092703.84-125433.7)

Ren ID 23410 (1SWASPJ093129.86-095912.9)

Ren ID 23672 (1SWASPJ093531.00-162820.1)

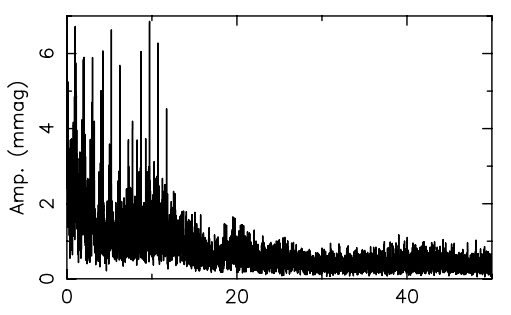

Freq. $\left(d^{-1}\right)$

Frequency $9.71984673 d^{-1}$

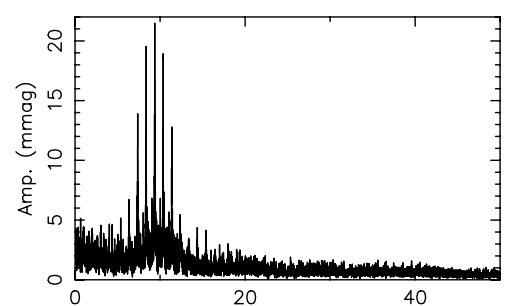

Freq. $\left(d^{-1}\right)$

Frequency $9.36723995 d^{-1}$

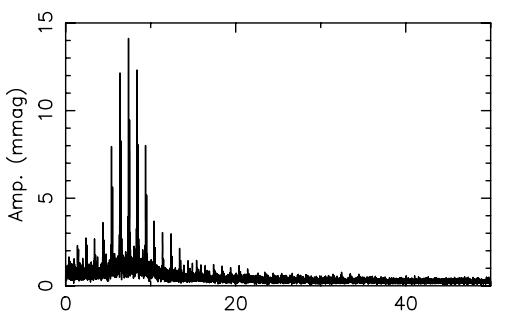

Freq. $\left(d^{-1}\right)$

Frequency $7.3911581 \mathrm{~d}^{-1}$
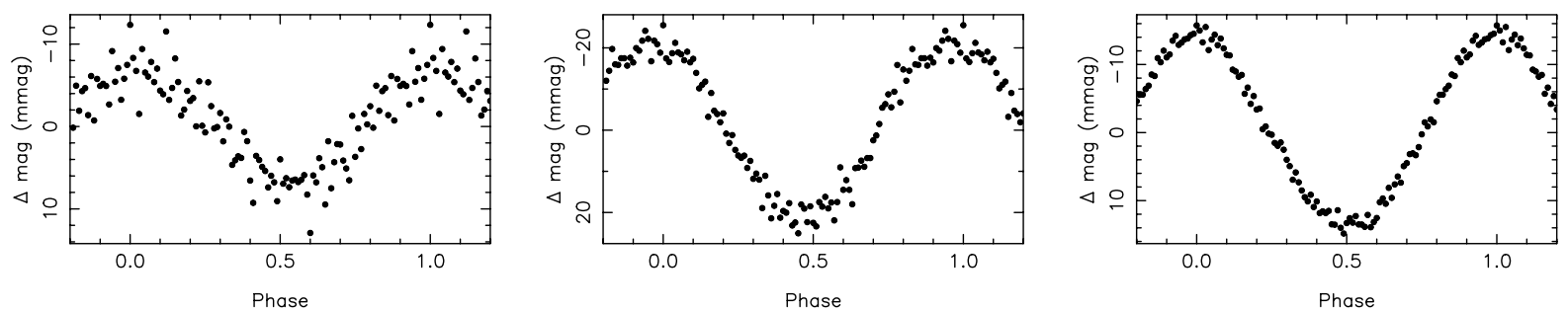

Fig. 1. continued. 
B. Smalley et al.: SuperWASP observations of pulsating Am stars

Ren ID 24920 (1SWASPJ100202.08-384115.0)

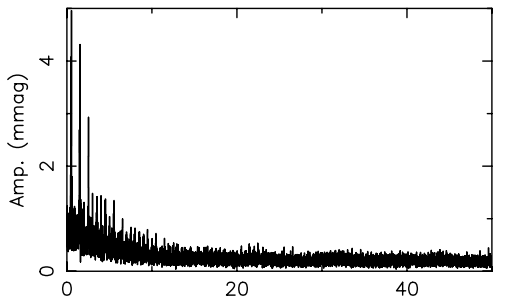

Freq. $\left(d^{-1}\right)$

Frequency $0.53337872 d^{-1}$

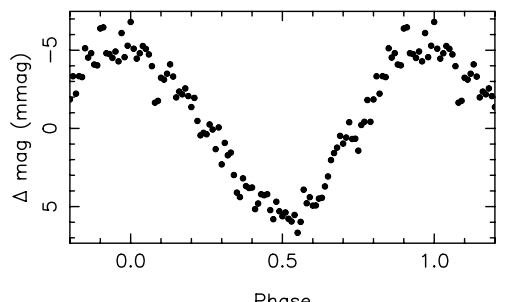

Ren ID 25730 (1SWASPJ102102.69+413650.2)

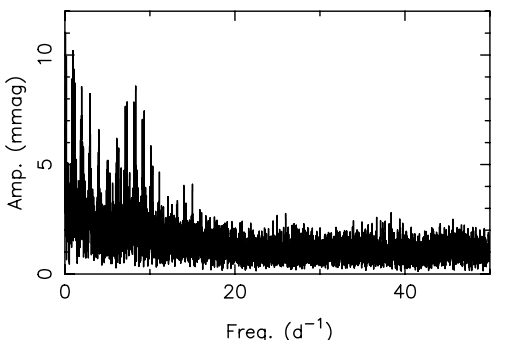

Frequency $8.32548141 \mathrm{~d}^{-1}$

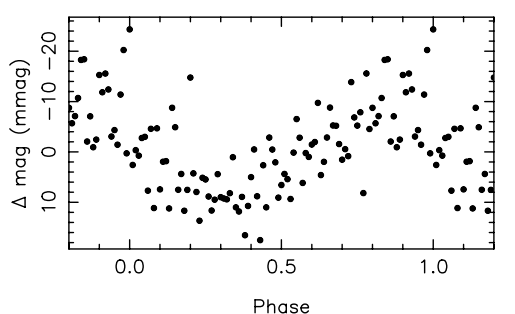

Ren ID 26880 (1SWASPJ104542.19+275754.1)

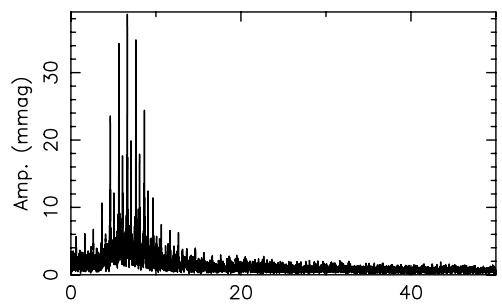

Freq. $\left(d^{-1}\right)$

Frequency $6.63842678 d^{-1}$

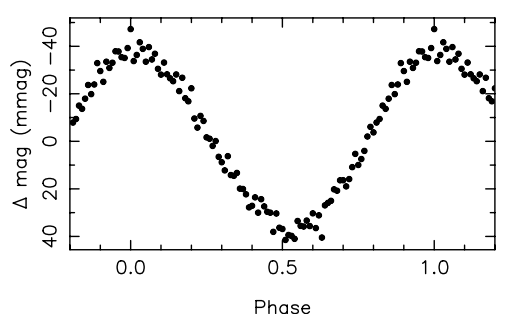

Ren ID 24990 (1SWASPJ100419.26-090941.8)

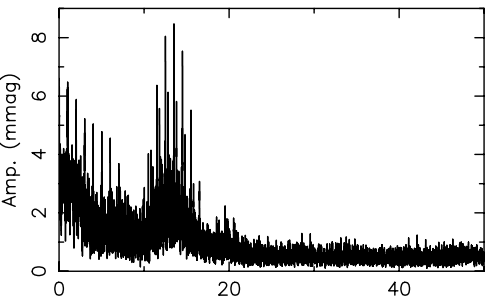

Freq. $\left(d^{-1}\right)$

Frequency $13.5112753 d^{-1}$

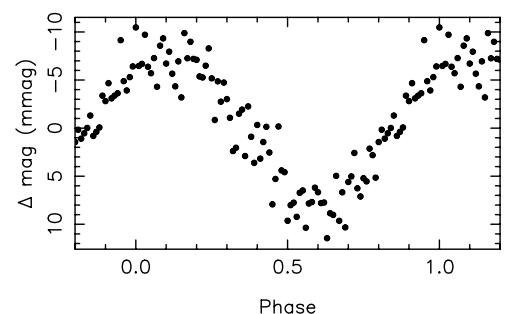

Ren ID 26360 (1SWASPJ103401.63-404025.0)

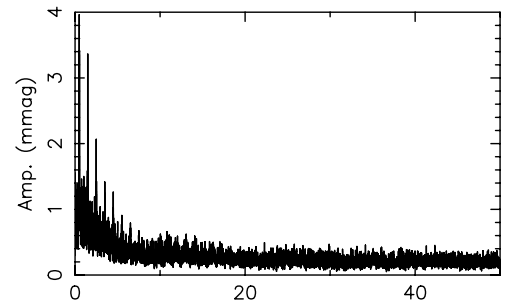

Freq. $\left(d^{-1}\right)$

Frequency $0.486142099 d^{-1}$

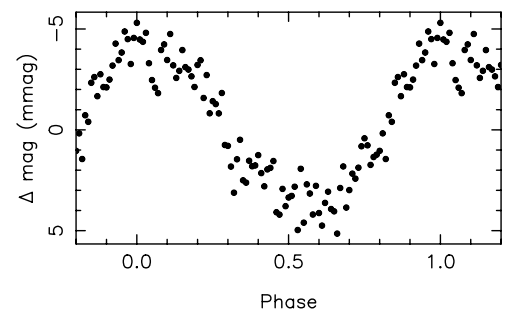

Ren ID 27270 (1SWASPJ105458.22+394726.1)

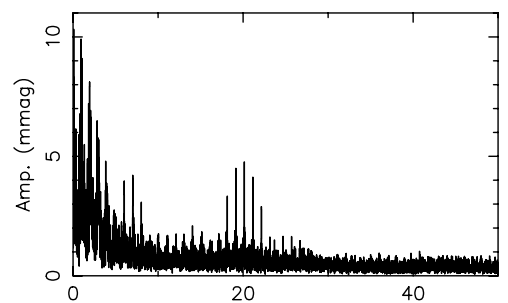

Freq. $\left(d^{-1}\right)$

Frequency $20.1477413 d^{-1}$

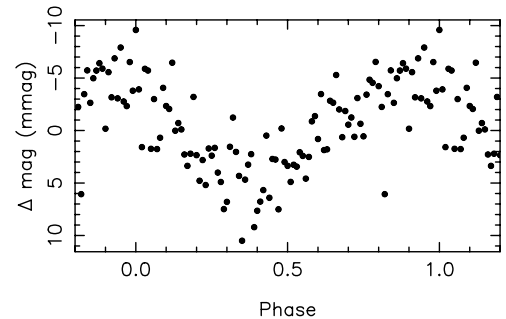

Ren ID 25160 (1SWASPJ100742.65-140954.6)

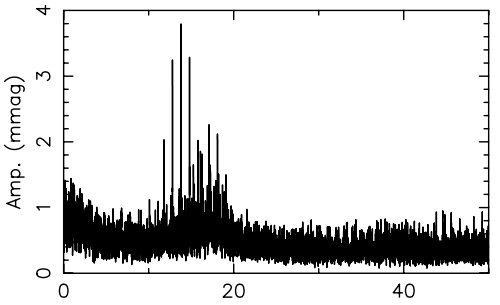

Freq. $\left(d^{-1}\right)$

Frequency $13.8007565 d^{-1}$

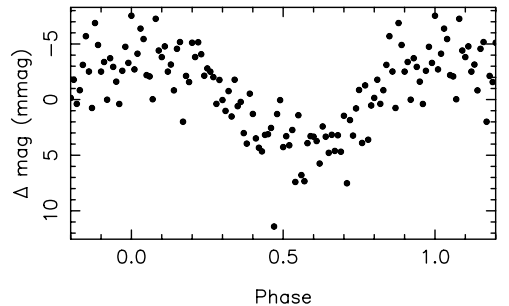

Ren ID 26860 (1SWASPJ104357.09-440512.8)

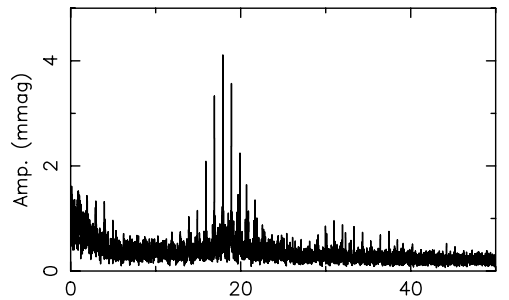

Freq. $\left(d^{-1}\right)$

Frequency $17.9073524 d^{-1}$

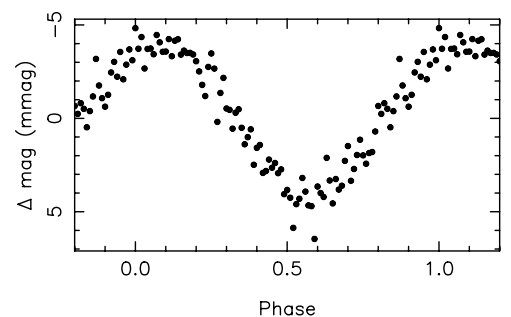

Ren ID 27405 (1SWASPJ105925.30-072037.9)

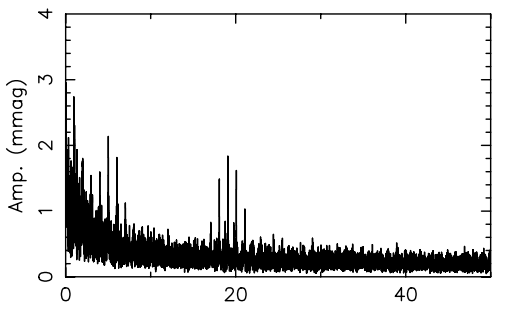

Freq. $\left(d^{-1}\right)$

Frequency $19.0771713 d^{-1}$

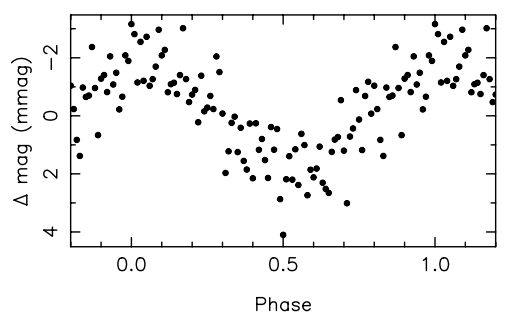

Fig. 1. continued. 


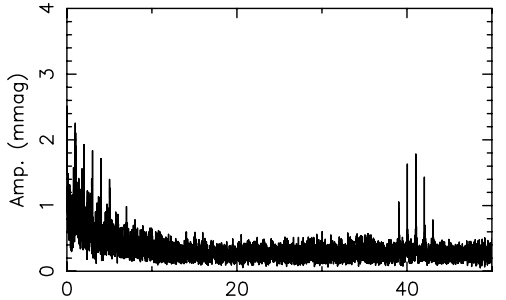

Freq. $\left(d^{-1}\right)$

Frequency $41.0463638 \mathrm{~d}^{-1}$

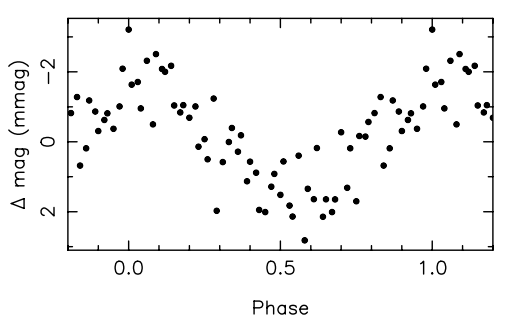

Ren ID 28340 (1SWASPJ111821.08-163305.0)

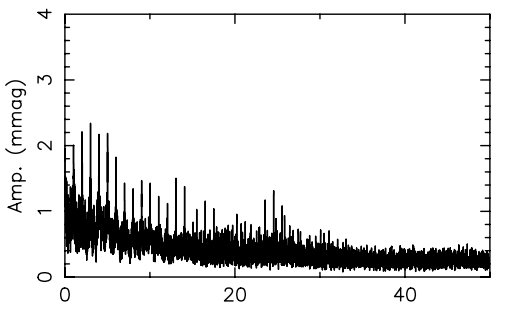

Freq. $\left(d^{-1}\right)$

Frequency $13.0735493 d^{-1}$

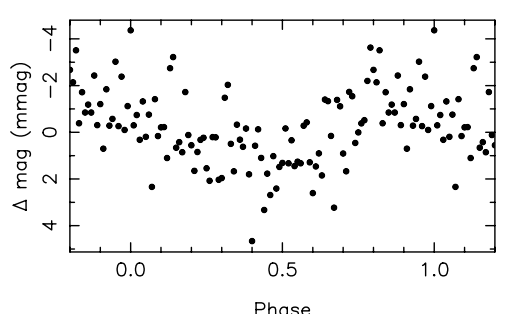

Ren ID 28690 (1SWASPJ112805.98-351156.1)

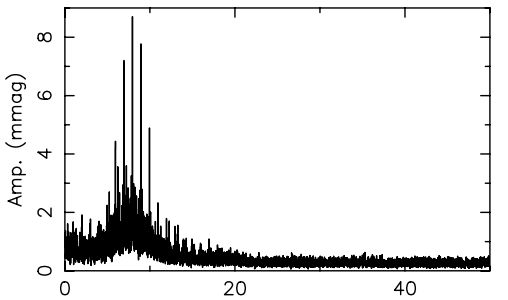

Freq. $\left(d^{-1}\right)$

Frequency $7.9437685 d^{-1}$

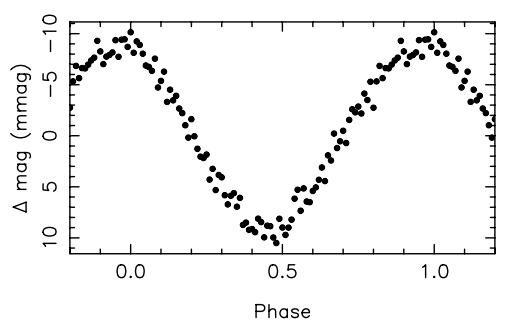

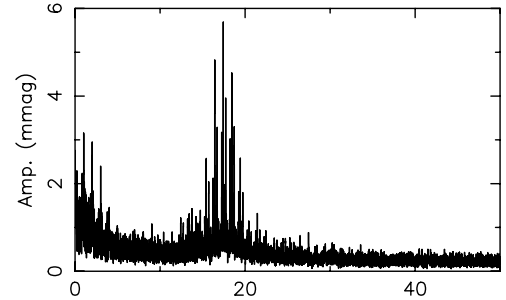

Freq. $\left(d^{-1}\right)$

Frequency $17.432888 d^{-1}$

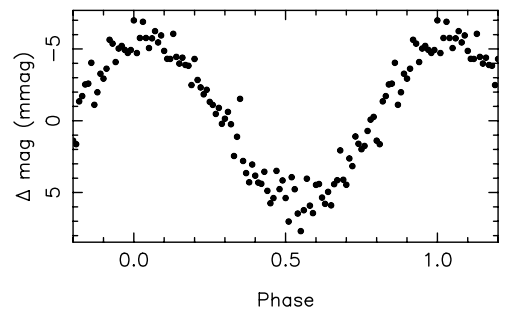

Ren ID 28510 (1SWASPJ112330.77+344914.7)

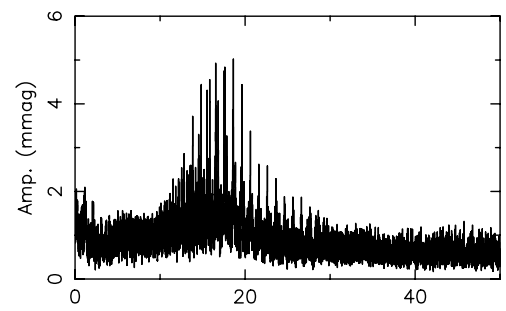

Freq. $\left(d^{-1}\right)$

Frequency $18.6186066 \mathrm{~d}^{-1}$

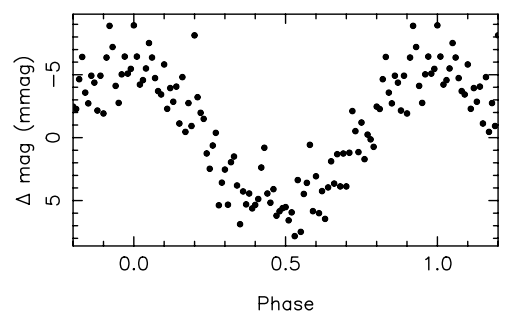

Ren ID 28850 (1SWASPJ113256.91-273723.4)

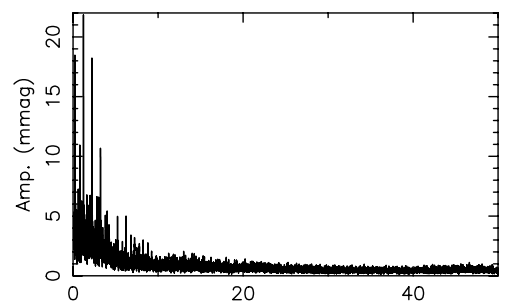

Freq. $\left(d^{-1}\right)$

Frequency $1.21685052 \mathrm{~d}^{-1}$

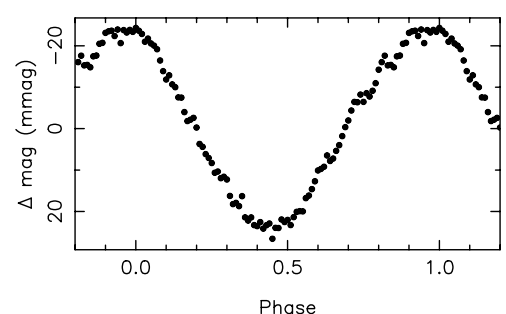

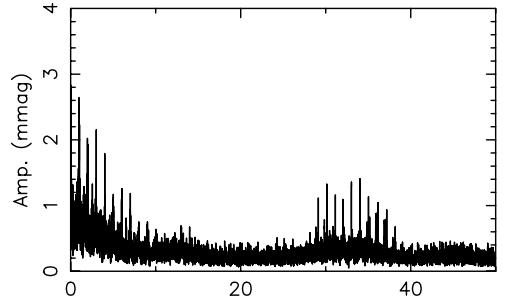

Freq. $\left(d^{-1}\right)$

Frequency $34.0299644 d^{-1}$

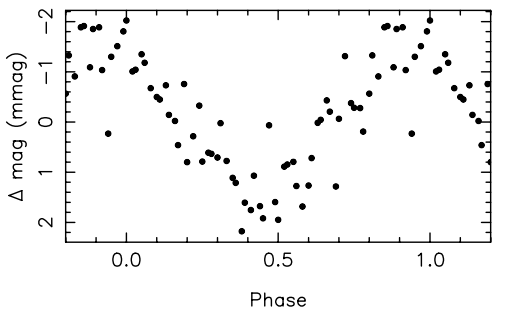

Ren ID 28610 (1SWASPJ112545.73+264450.0)

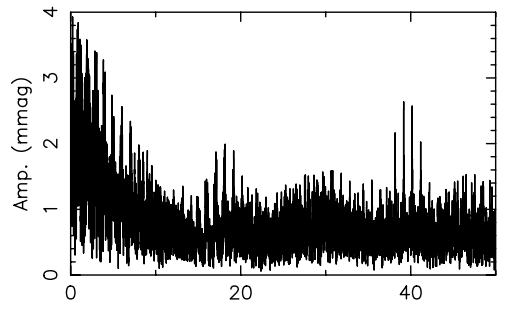

Freq. $\left(d^{-1}\right)$

Frequency $39.174263 d^{-1}$

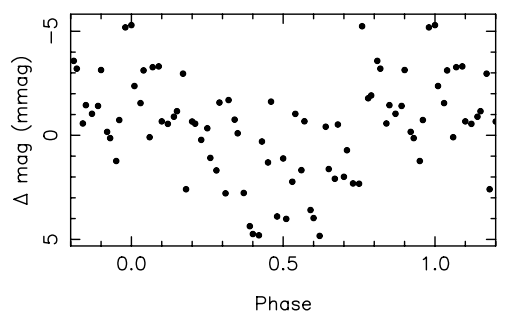

Ren ID 29280 (1SWASPJ114130.23+403822.7)

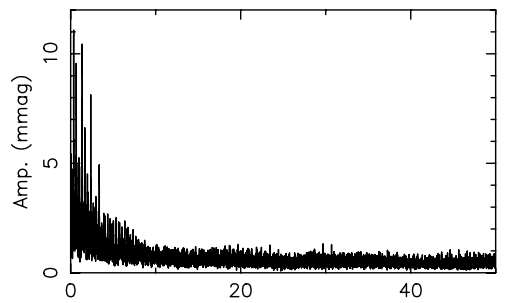

Freq. $\left(d^{-1}\right)$

Frequency $0.350461096 d^{-1}$

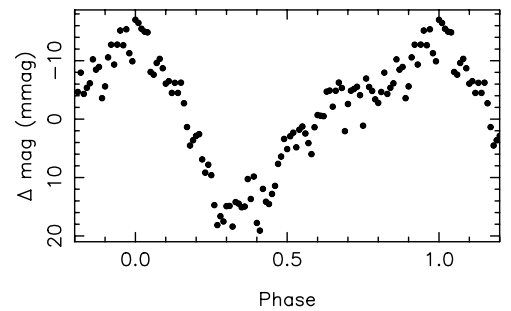

Fig. 1. continued. 
B. Smalley et al.: SuperWASP observations of pulsating Am stars

Ren ID 29310 (1SWASPJ114215.76-154343.0)

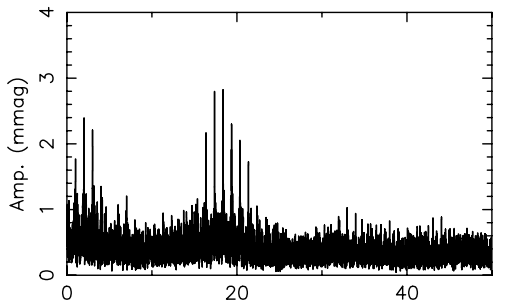

Freq. $\left(d^{-1}\right)$

Frequency $18.3662891 \mathrm{~d}^{-1}$

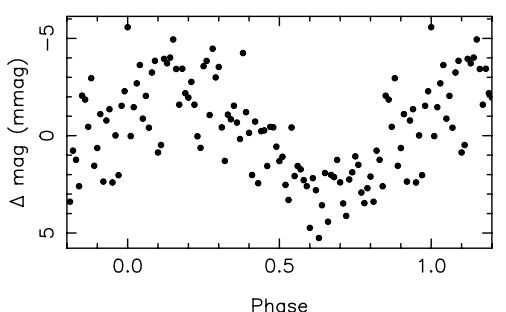

Ren ID 30390 (1SWASPJ120506.30+304957.1)

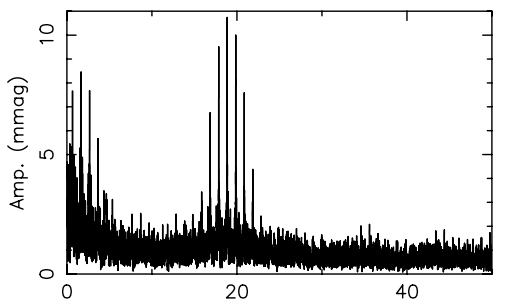

Freq. $\left(d^{-1}\right)$

Frequency $18.8594418 d^{-1}$

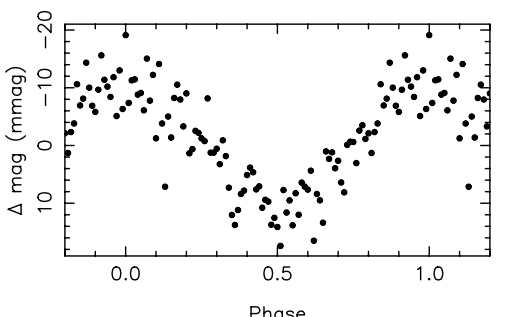

Ren ID 30970 (1SWASPJ121828.73+344052.3)

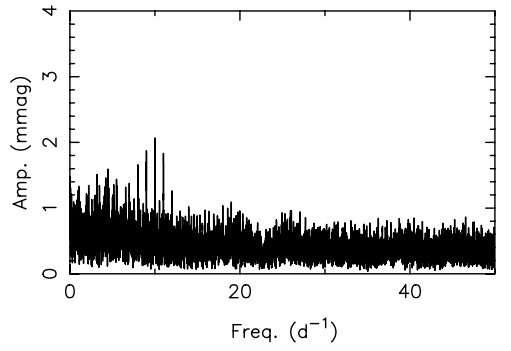

Frequency $10.000948 d^{-1}$

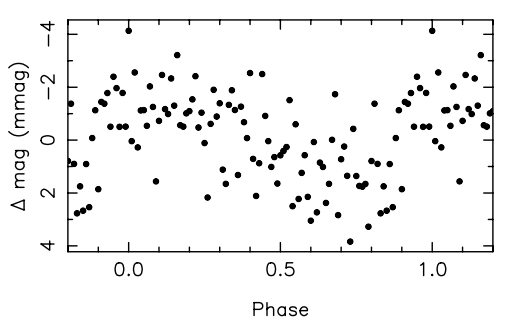

Ren ID 29590 (1SWASPJ114827.17-315807.4)

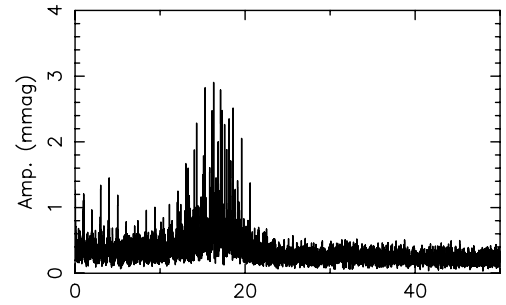

Freq. $\left(d^{-1}\right)$

Frequency $16.3002625 d^{-1}$

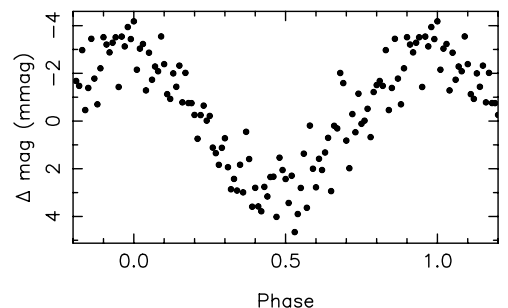

Ren ID 30453 (1SWASPJ120728.65+172344.0)

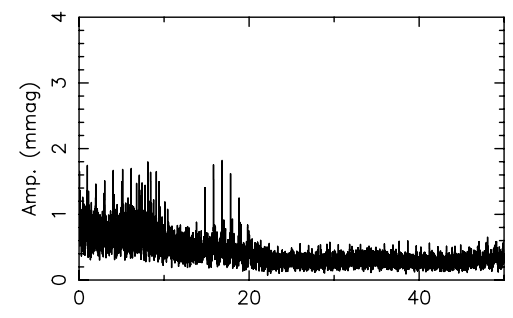

Freq. $\left(d^{-1}\right)$

Frequency $16.8163109 d^{-1}$

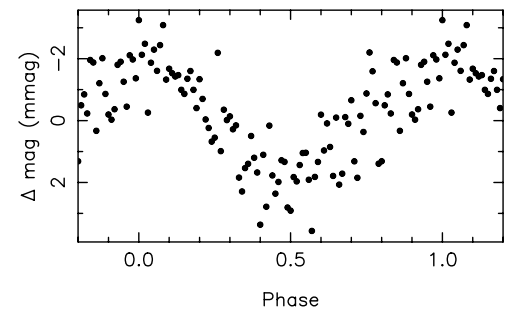

Ren ID 31500 (1SWASPJ122732.77+111648.6)

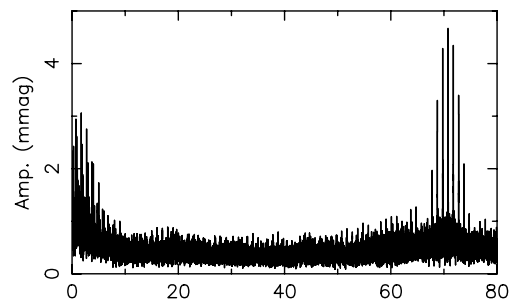

Freq. $\left(d^{-1}\right)$

Frequency $70.7600174 d^{-1}$

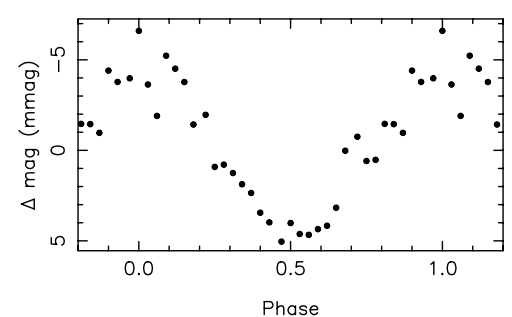

Ren ID 29800 (1SWASPJ115340.75-371804.9)

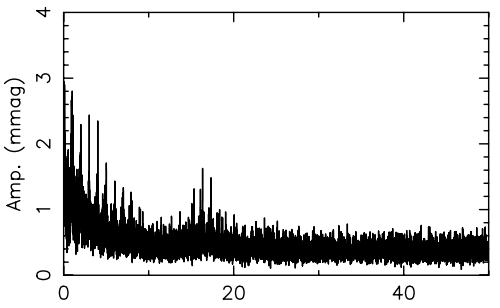

Freq. $\left(d^{-1}\right)$

Frequency $16.3386345 d^{-1}$

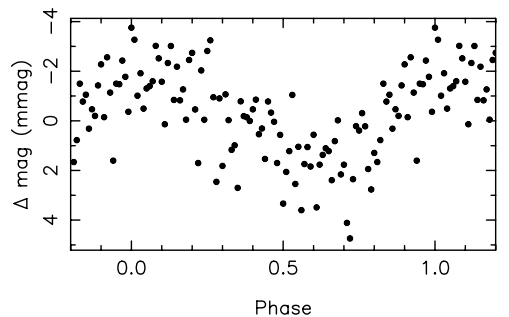

Ren ID 30926 (1SWASPJ121707.63+014245.0)

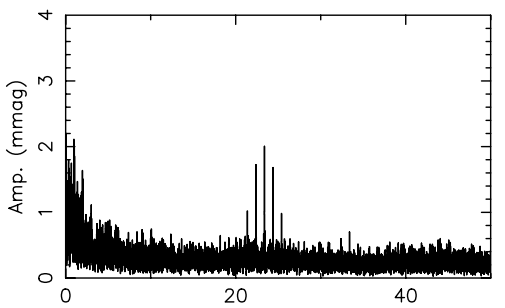

Freq. $\left(d^{-1}\right)$

Frequency $23.383625 \mathrm{~d}^{-1}$

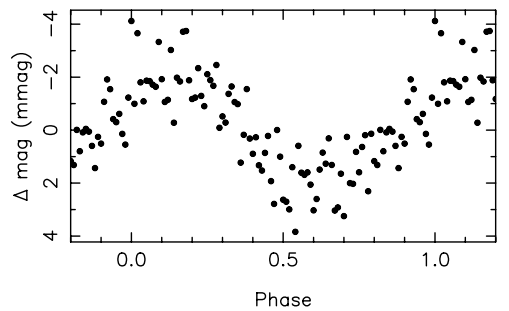

Ren ID 31560 (1SWASPJ122916.13-365652.2)

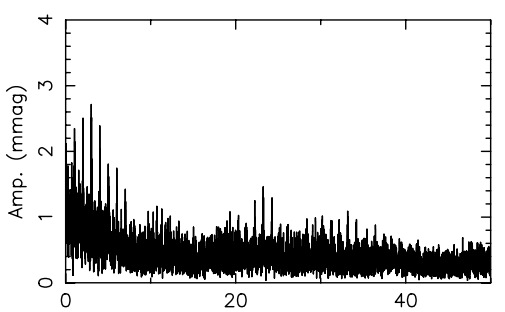

Freq. $\left(d^{-1}\right)$

Frequency $23.2409821 d^{-1}$

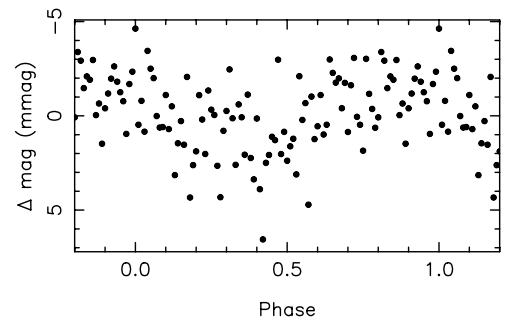

Fig. 1. continued. 


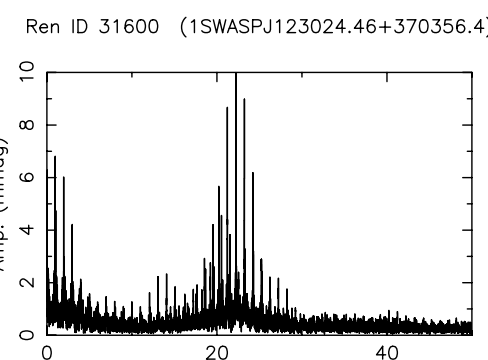

Freq. $\left(d^{-1}\right)$

Frequency $22.2298126 d^{-1}$

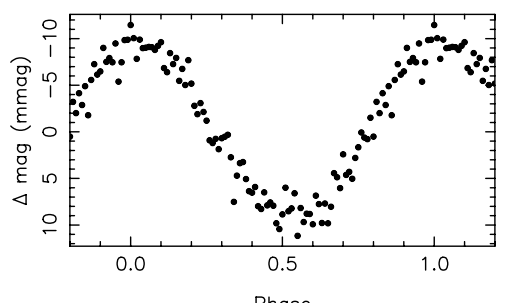

Ren ID 31800 (1SWASPJ123618.42+352224.9)

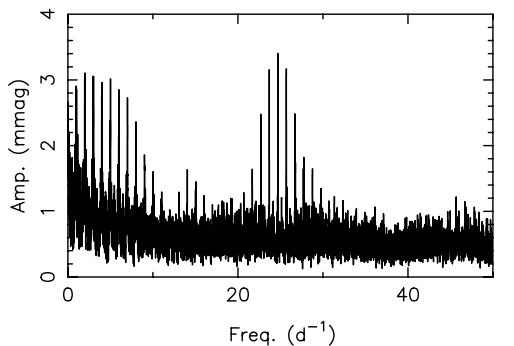

Frequency $24.6919365 d^{-1}$

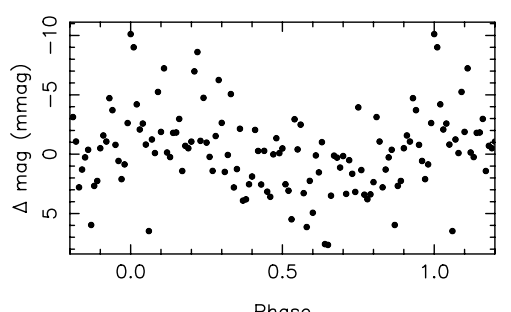

Ren ID 32180 (1SWASPJ124309.47+380339.3)

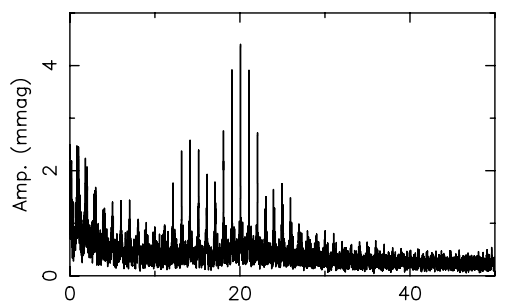

Freq. $\left(d^{-1}\right)$

Frequency $20.0646152 d^{-1}$

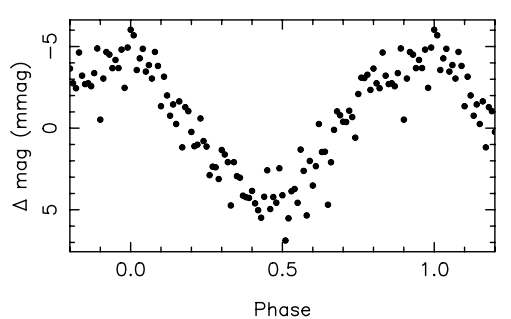

Ren ID 31680 (1SWASPJ123318.88+391049.0)

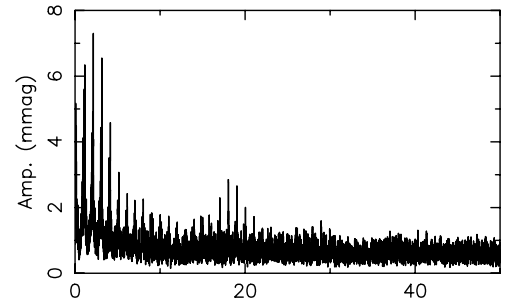

Freq. $\left(d^{-1}\right)$

Frequency $2.14287329 d^{-1}$

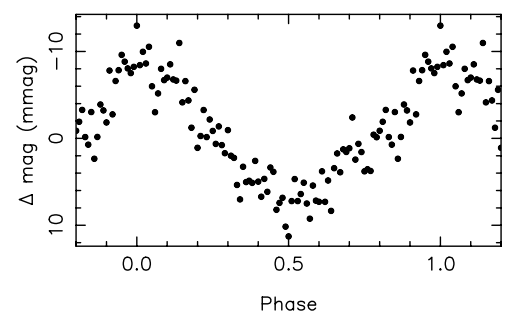

Ren ID 31913 (1SWASPJ123847.64-031832.6)

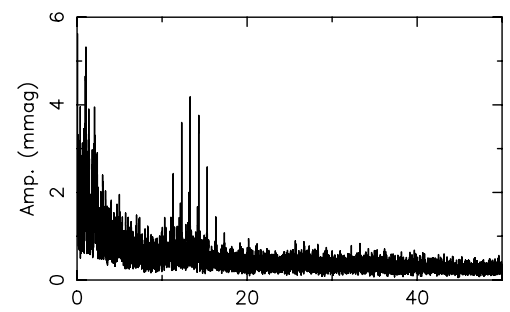

Freq. $\left(d^{-1}\right)$

Frequency $13.3080397 d^{-1}$

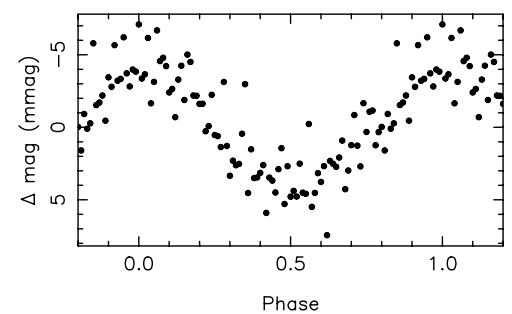

Ren ID 32340 (1SWASPJ124816.41+205245.6)

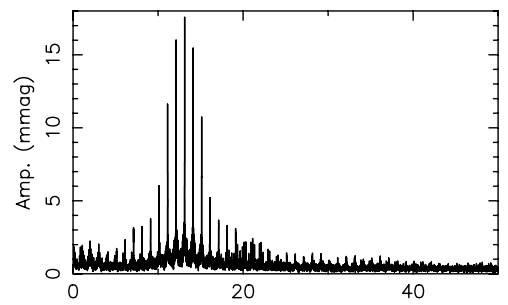

Freq. $\left(d^{-1}\right)$

Frequency $13.1200466 d^{-1}$

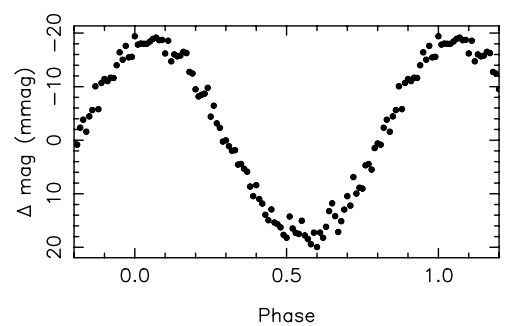

Ren ID 31710 (1SWASPJ123336.95+280453.3)

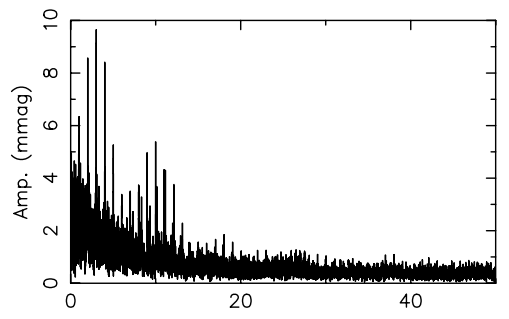

Freq. $\left(d^{-1}\right)$

Frequency $3.0067606 \mathrm{~d}^{-1}$

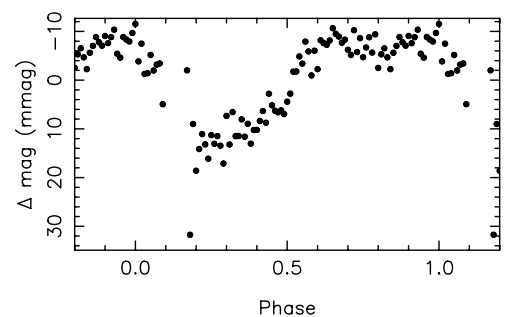

Ren ID 31950 (1SWASPJ123940.56-422227.9)

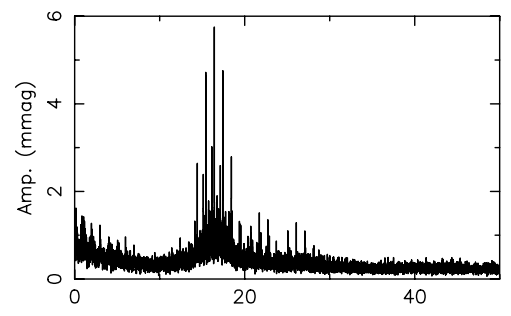

Freq. $\left(d^{-1}\right)$

Frequency $16.4269047 d^{-1}$

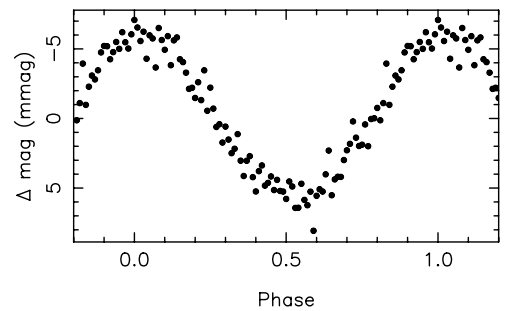

Ren ID 32624 (1SWASPJ125557.11+161339.8)

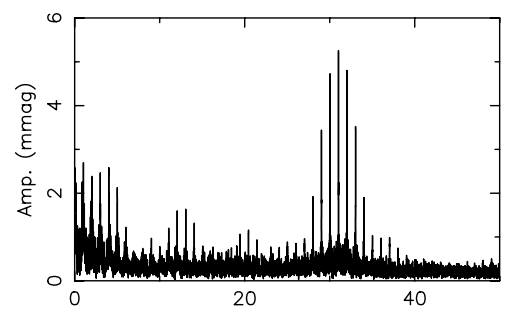

Freq. $\left(d^{-1}\right)$

Frequency $31.0375729 d^{-1}$

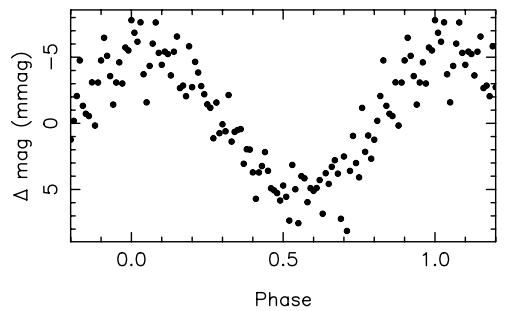

Fig. 1. continued. 
B. Smalley et al.: SuperWASP observations of pulsating Am stars

Ren ID 32870 (1SWASPJ130243.35-411358.8)

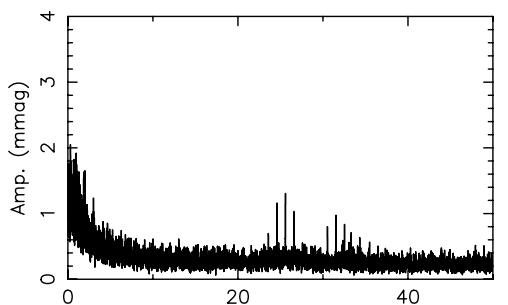

Freq. $\left(d^{-1}\right)$

Frequency $25.5893364 d^{-1}$

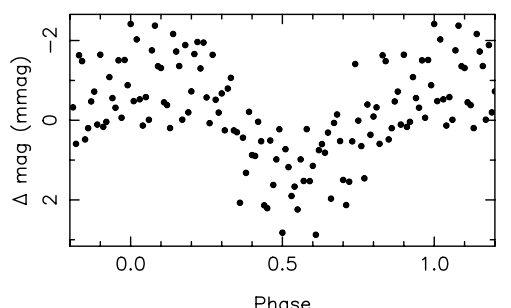

Ren ID 33490 (1SWASPJ131953.62-274815.8)

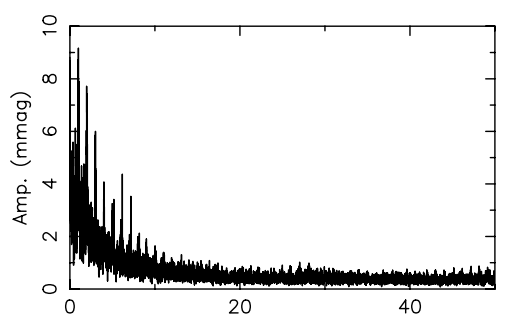

Freq. $\left(d^{-1}\right)$

Frequency $6.16544962 d^{-1}$

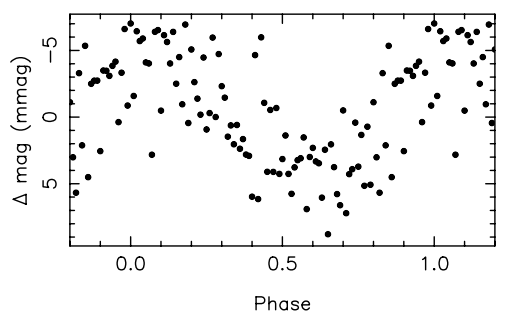

Ren ID 33940 (1SWASPJ133235.84-364441.9)

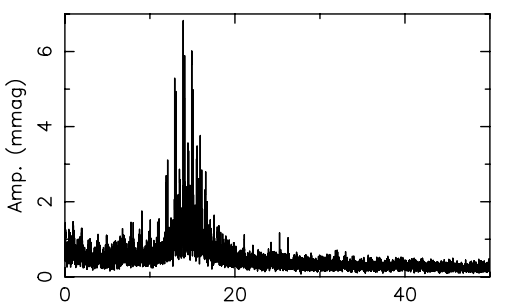

Freq. $\left(d^{-1}\right)$

Frequency $13.9188976 d^{-1}$

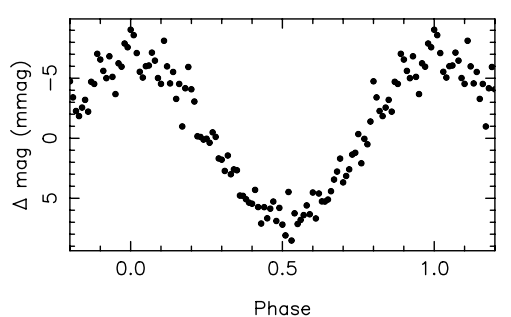

Ren ID 32885 (1SWASPJ130328.44-194035.1)

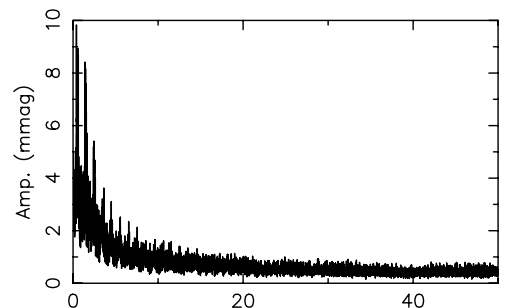

Freq. $\left(d^{-1}\right)$

Frequency $0.390450776 d^{-1}$

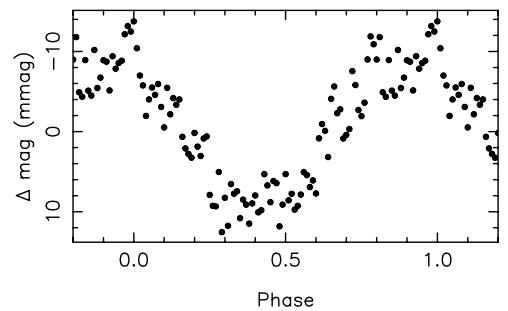

Ren ID 33555 (1SWASPJ132255.12-181203.4)

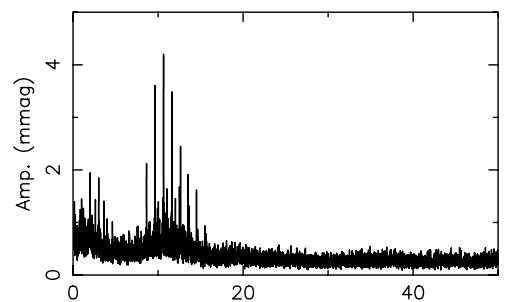

Freq. $\left(d^{-1}\right)$

Frequency $10.6403637 d^{-1}$

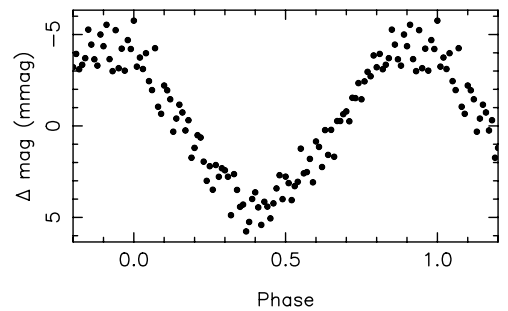

Ren ID 34137 (1SWASPJ133523.75+351339.0)

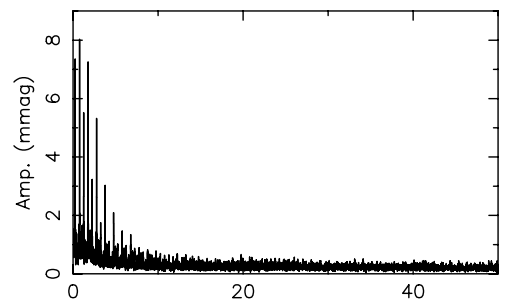

Freq. $\left(d^{-1}\right)$

Frequency $0.760733724 d^{-1}$

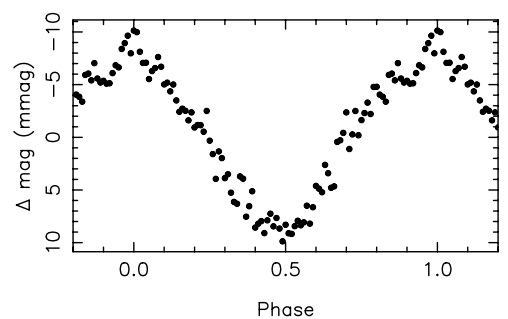

Ren ID 33220 (1SWASPJ131247.43+265252.1)

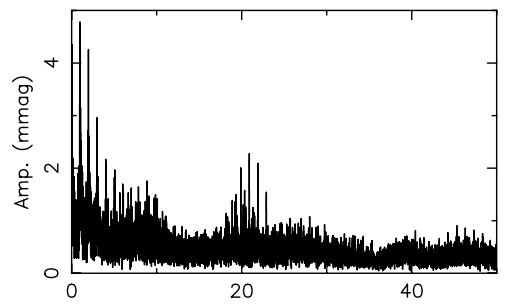

Freq. $\left(d^{-1}\right)$

Frequency $0.966495514 d^{-1}$

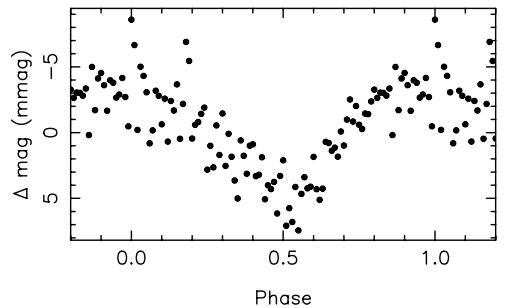

Ren ID 33636 (1SWASPJ132426.36+303314.2)

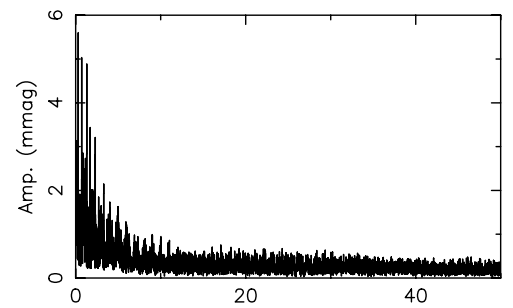

Freq. $\left(d^{-1}\right)$

Frequency $0.299687177 d^{-1}$

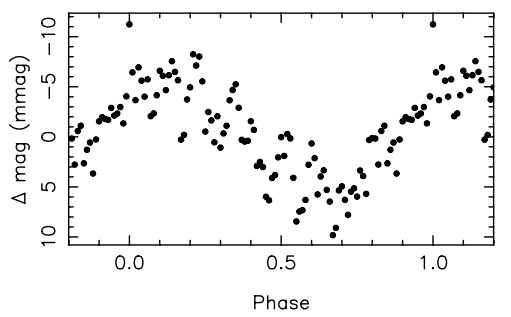

Ren ID 34076 (1SWASPJ133534.05-144948.4)

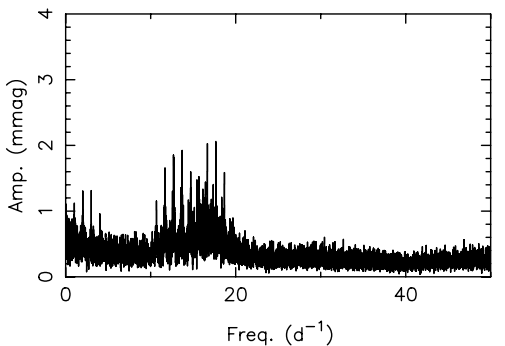

Frequency $17.6727486 d^{-1}$

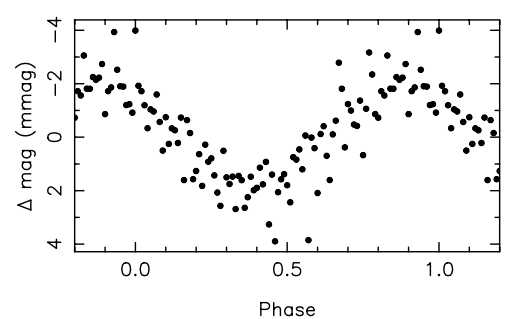

Fig. 1. continued. 
A\&A 535, A3 (2011)

Ren ID 34620 (1SWASPJ134749.25-332520.4)

Ren ID 34930 (1SWASPJ135527.34-273842.2)

Ren ID 34920 (1SWASPJ135535.64-430731.3)
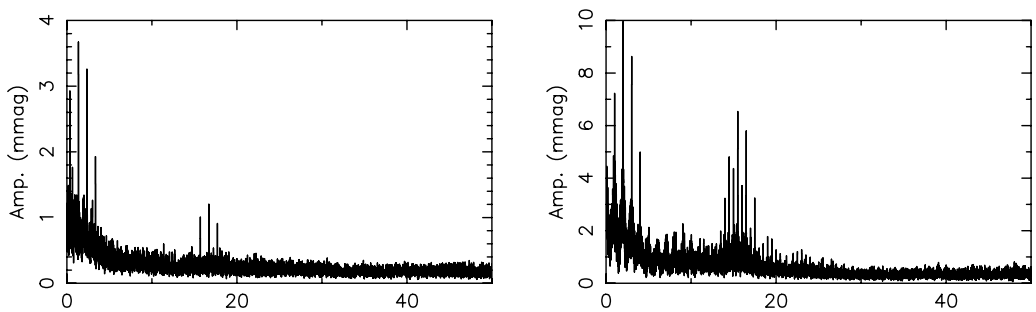

Freq. $\left(d^{-1}\right)$

Freq. $\left(d^{-1}\right)$

Frequency $1.35389304 \mathrm{~d}^{-1}$

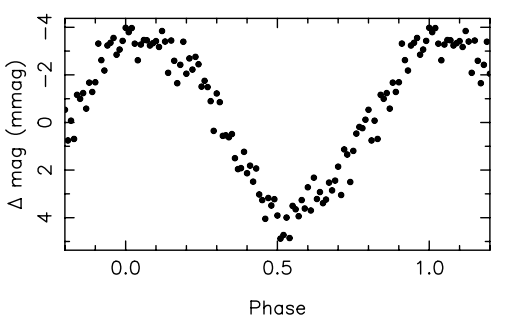

Frequency $15.4849453 d^{-1}$

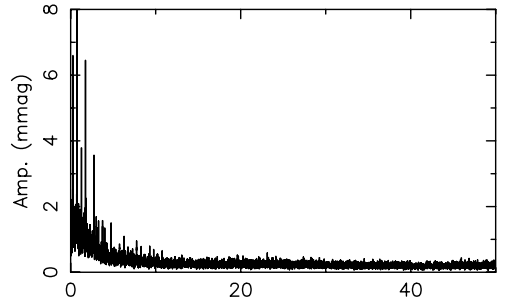

Freq. $\left(d^{-1}\right)$

Frequency $0.736288607 \mathrm{~d}^{-1}$
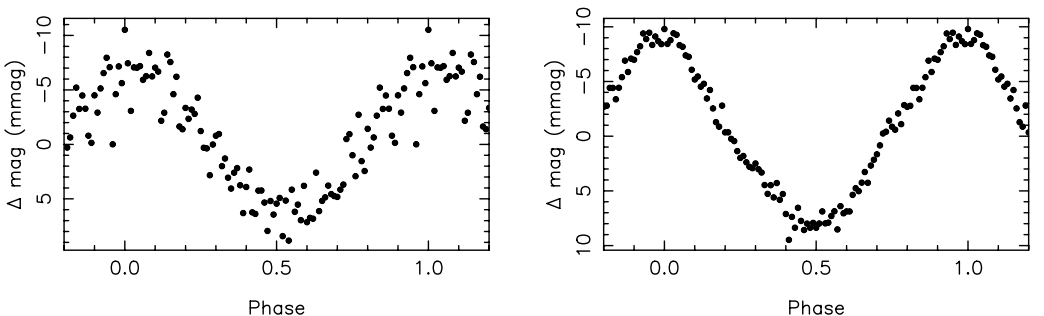

Ren ID 34996 (1SWASPJ135726.20-221853.2)

Ren ID 35074 (1SWASPJ140138.31-095624.4)

Ren ID 35450 (1SWASPJ141141.05-434456.5)

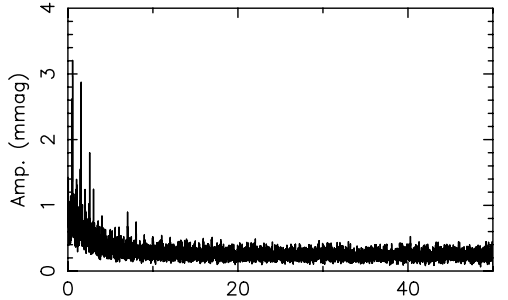

Freq. $\left(d^{-1}\right)$

Frequency $0.549834847 d^{-1}$
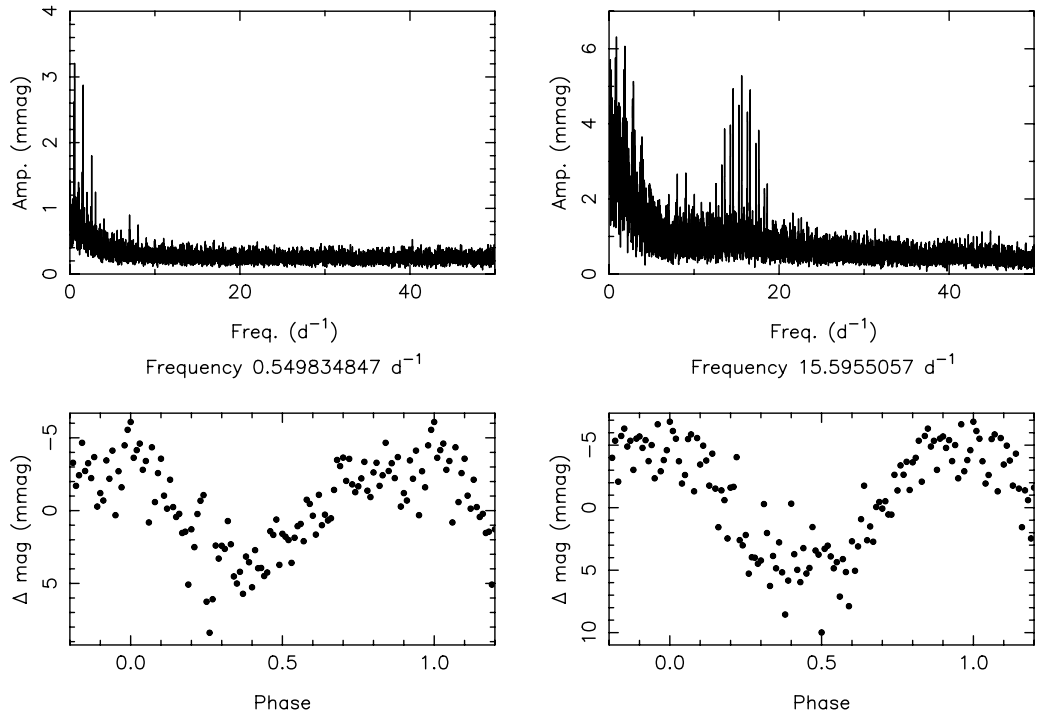

Freq. $\left(d^{-1}\right)$

Frequency $15.5955057 \mathrm{~d}^{-1}$
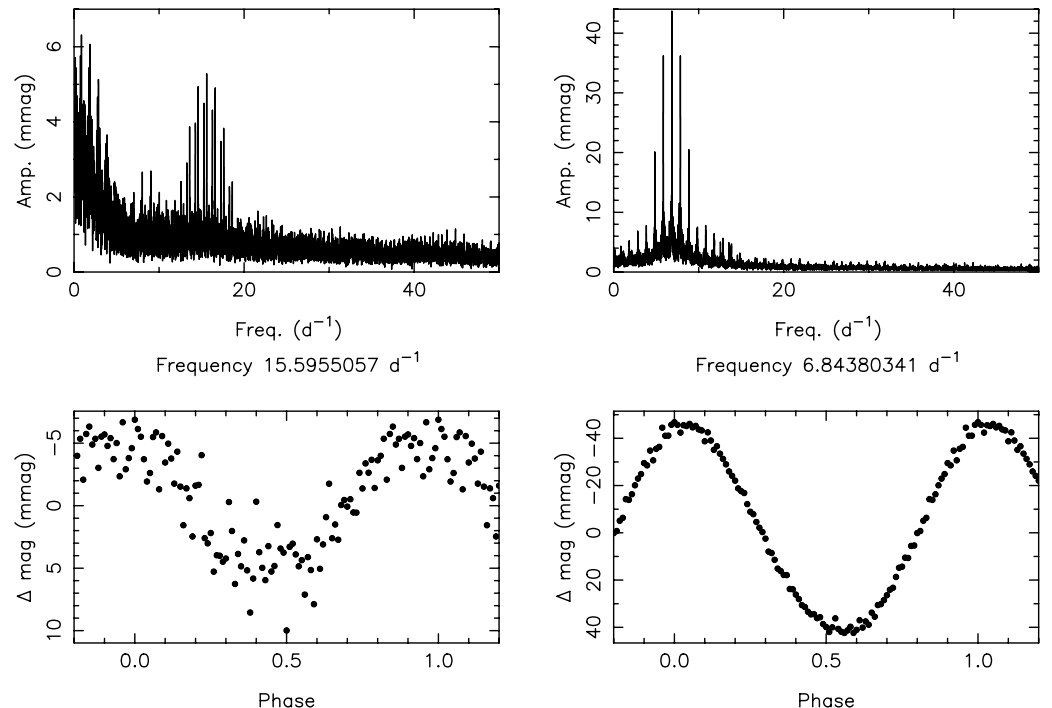

Freq. $\left(d^{-1}\right)$

Frequency $6.84380341 \mathrm{~d}^{-1}$

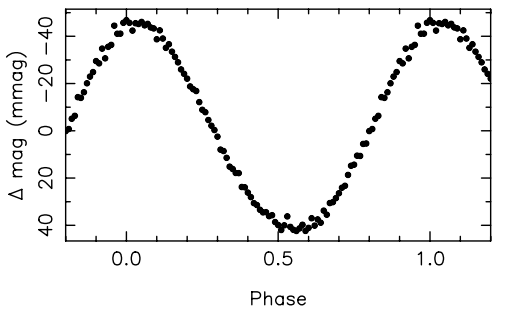

Ren ID 35650 (1SWASPJ141437.06-333336.0)

Ren ID 35710 (1SWASPJ141709.31-391707.4)

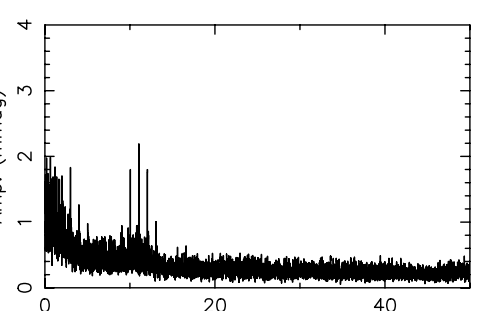

Freq. $\left(d^{-1}\right)$

Frequency $11.0526066 \mathrm{~d}^{-1}$
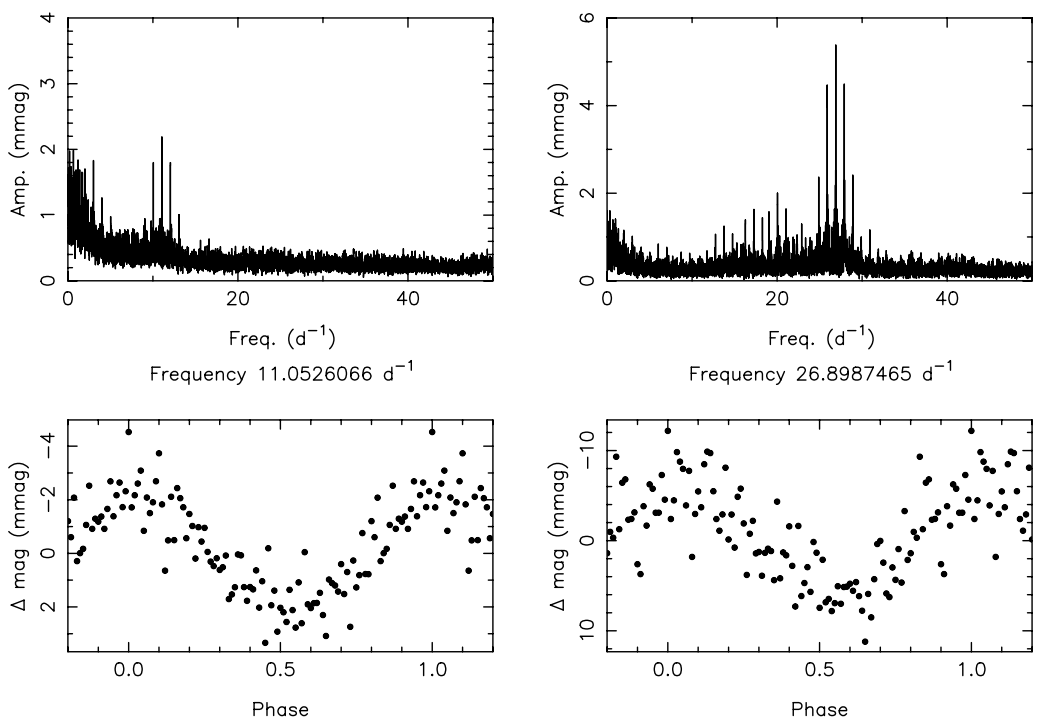

Freq. $\left(d^{-1}\right)$

Frequency $26.8987465 d^{-1}$
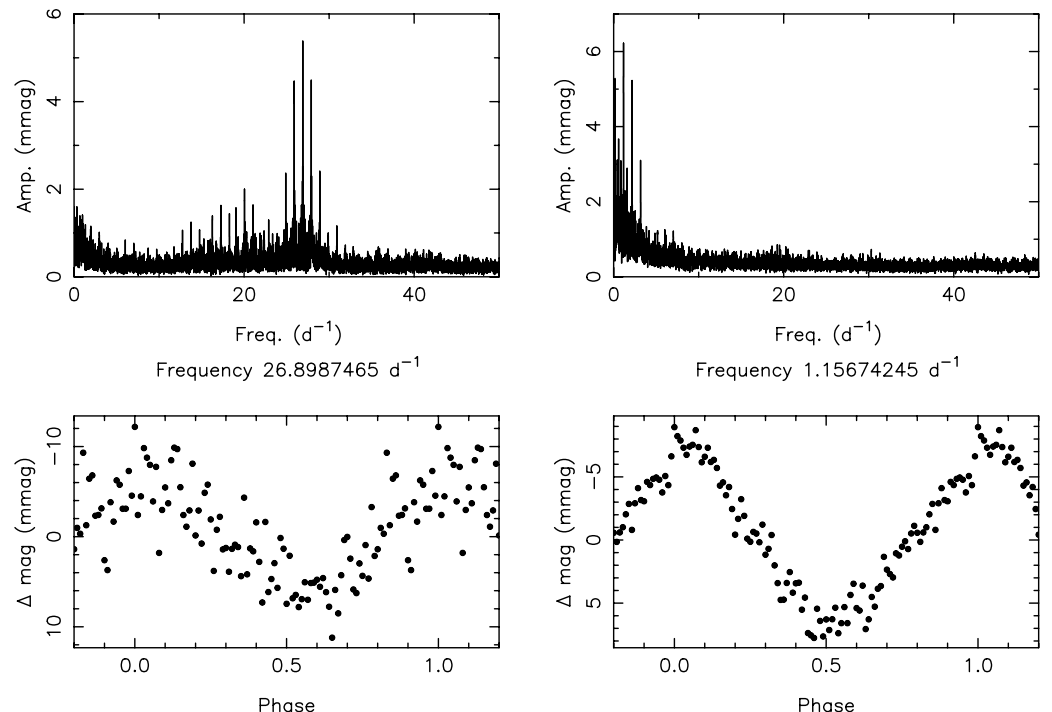

Freq. $\left(d^{-1}\right)$

Frequency $1.15674245 d^{-1}$

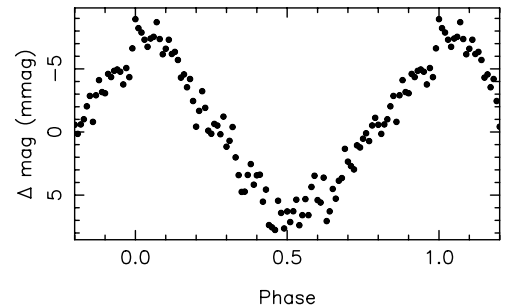

Fig. 1. continued. 
B. Smalley et al.: SuperWASP observations of pulsating Am stars

Ren ID 35776 (1SWASPJ141847.21-123500.5)

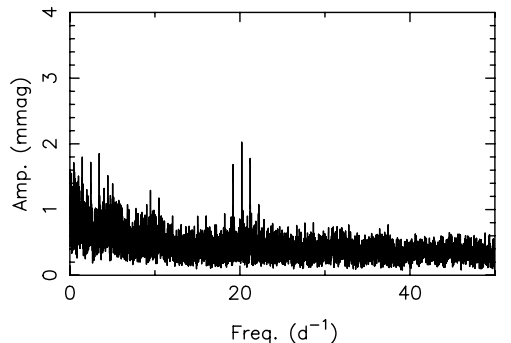

Frequency $20.210844 d^{-1}$

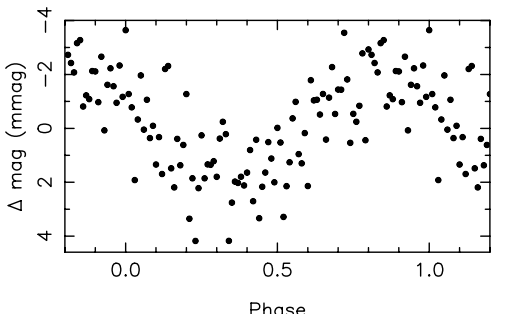

Ren ID 36940 (1SWASPJ144351.68-264043.4)

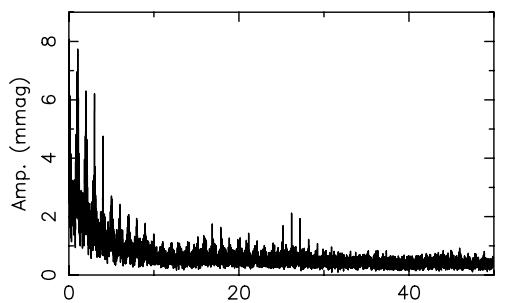

Freq. $\left(d^{-1}\right)$

Frequency $26.1936245 d^{-1}$

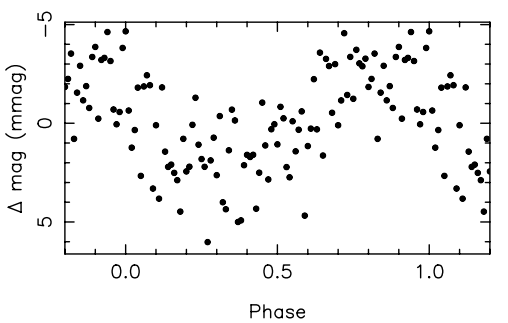

Ren ID 37884 (1SWASPJ150517.36-193616.7)

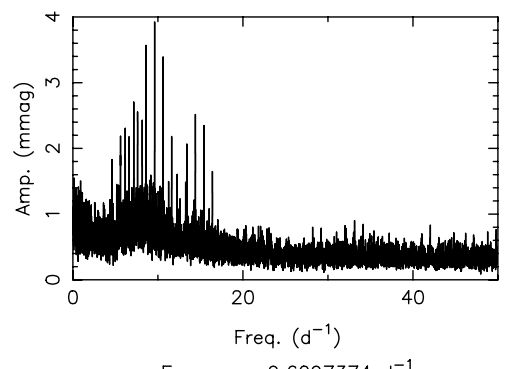

Frequency $9.6097374 d^{-1}$

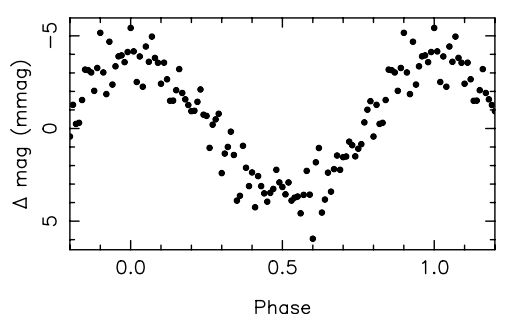

Ren ID 36080 (1SWASPJ142745.12-295858.6)

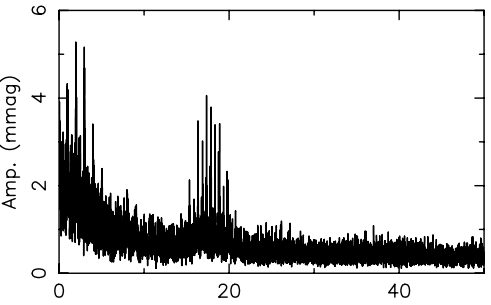

Freq. $\left(d^{-1}\right)$

Frequency $17.3455696 d^{-1}$

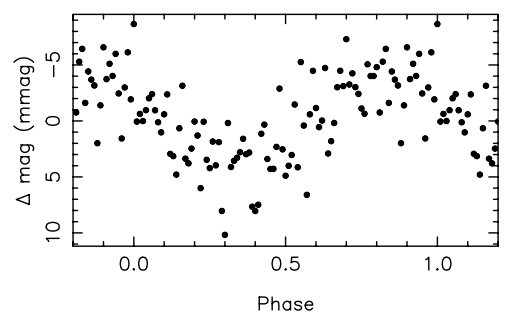

Ren ID 37513 (1SWASPJ145724.43-012550.3)

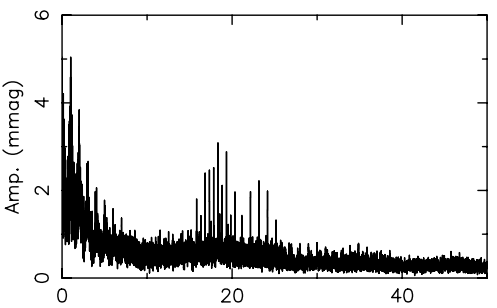

Freq. $\left(d^{-1}\right)$

Frequency $18.3348312 d^{-1}$

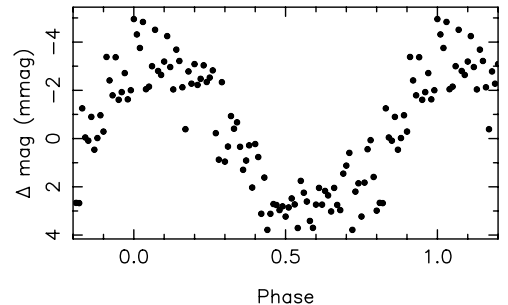

Ren ID 38400 (1SWASPJ151555.71-425038.1)

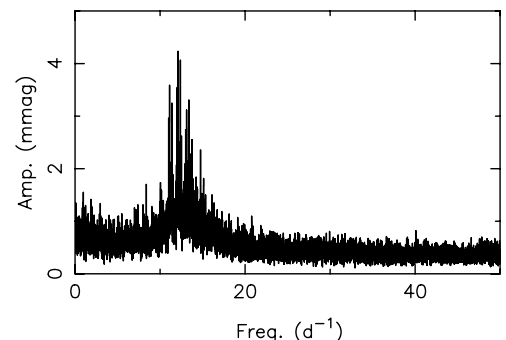

Frequency $12.1155987 d^{-1}$

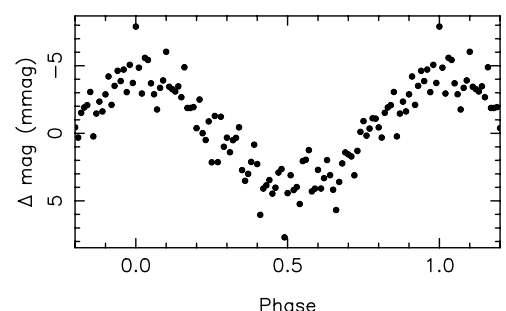

Ren ID 36330 (1SWASPJ143435.48-381221.9)

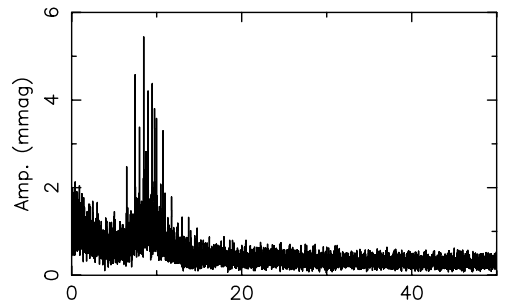

Freq. $\left(d^{-1}\right)$

Frequency $8.47139072 d^{-1}$

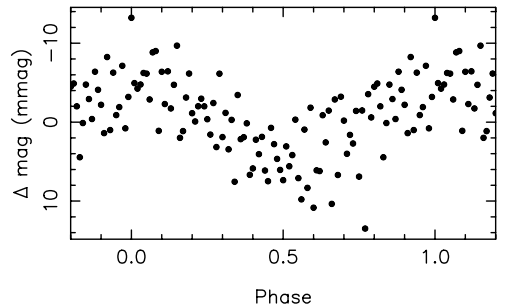

Ren ID 37494 (1SWASPJ145734.72-175550.3)

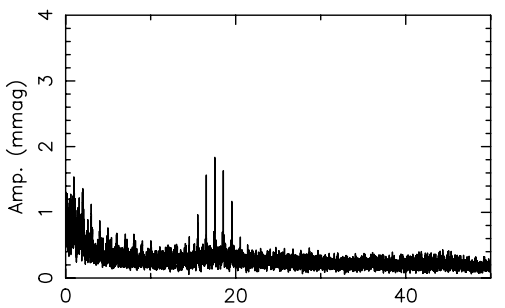

Freq. $\left(d^{-1}\right)$

Frequency $17.542799 \mathrm{~d}^{-1}$

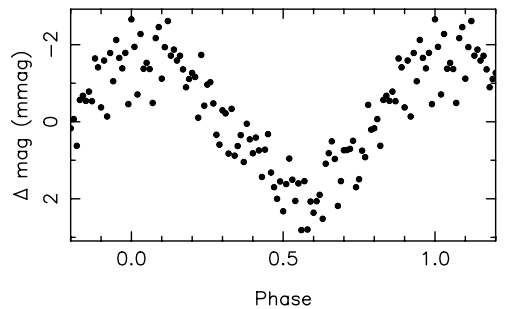

Ren ID 40280 (1SWASPJ155301.60-301504.4)

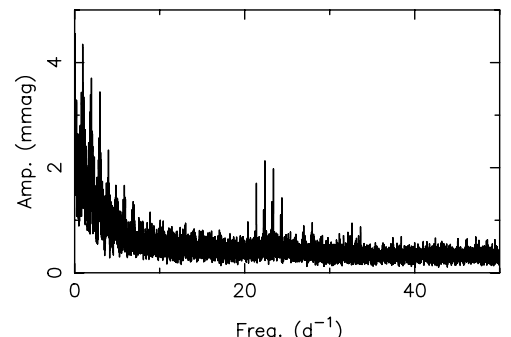

Frequency $22.3806896 d^{-1}$

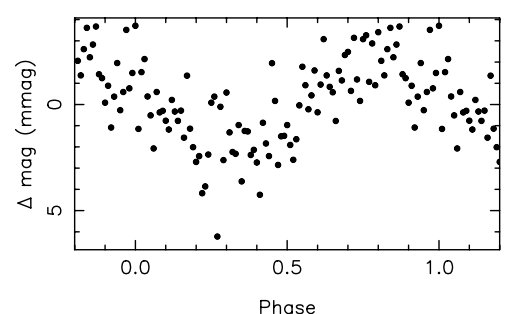

Fig. 1. continued. 


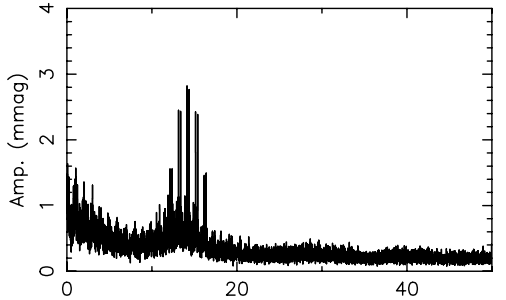

Freq. $\left(d^{-1}\right)$

Frequency $14.1302567 d^{-1}$

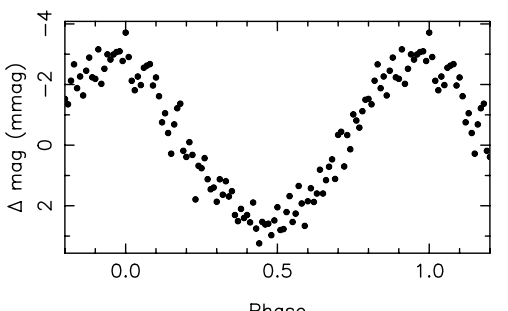

Ren ID 41030 (1SWASPJ160839.69-285120.6)

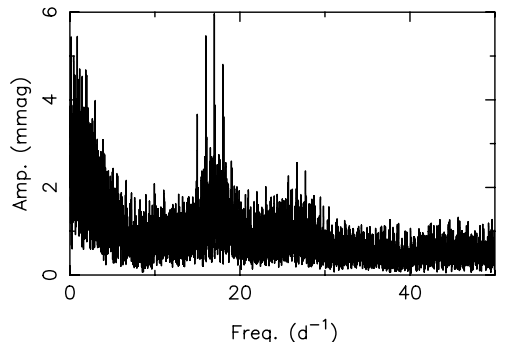

Frequency $16.988472 \mathrm{~d}^{-1}$

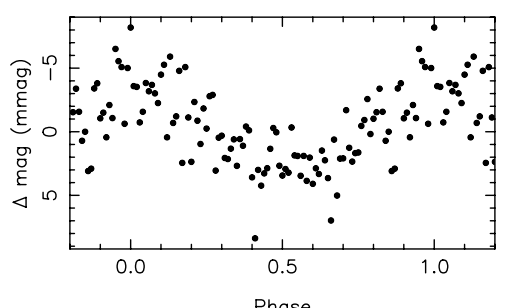

Ren ID 43588 (1SWASPJ170221.20+400422.8)

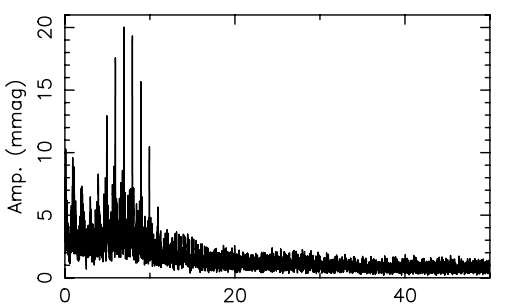

Freq. $\left(d^{-1}\right)$

Frequency $6.93457651 \mathrm{~d}^{-1}$

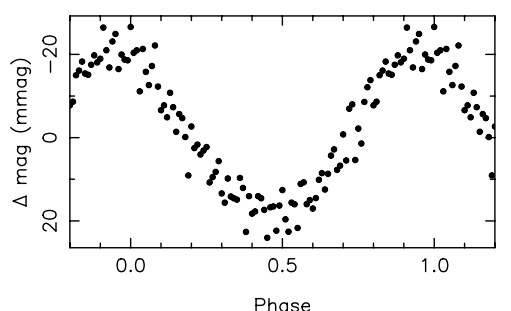

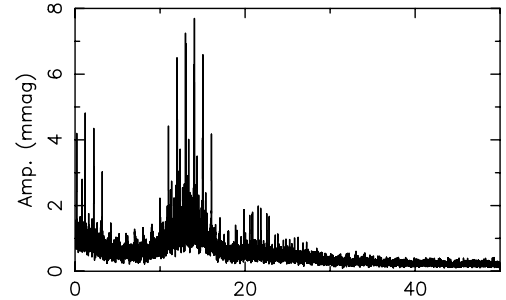

Freq. $\left(d^{-1}\right)$

Frequency $14.0384455 d^{-1}$

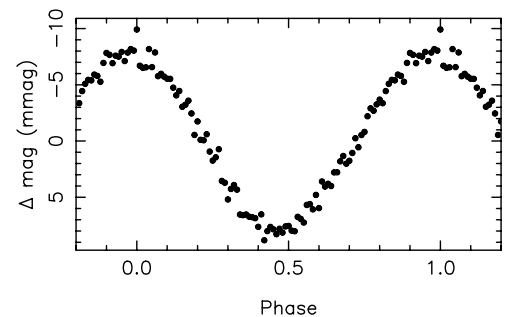

Ren ID 41315 (1SWASPJ161500.45-200249.9)

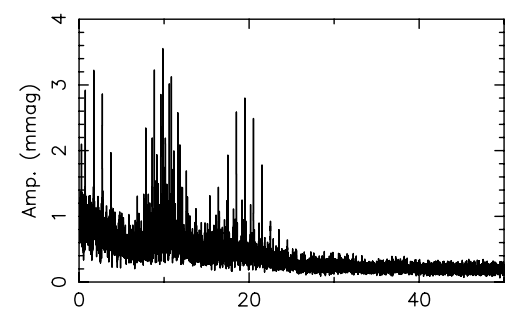

Freq. $\left(d^{-1}\right)$

Frequency $9.85326767 d^{-1}$

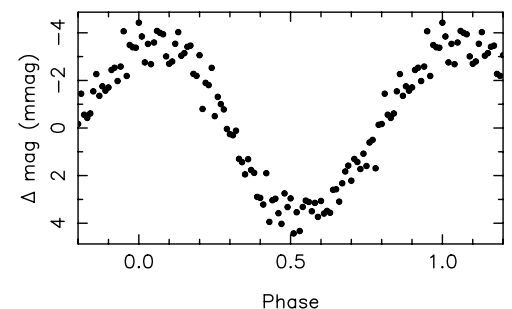

Ren ID 43590 (1SWASPJ170253.25+314124.6)

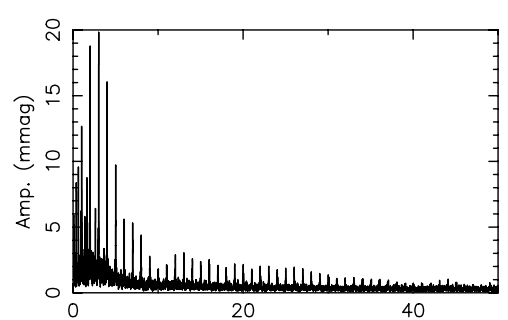

Freq. $\left(d^{-1}\right)$

Frequency $0.623309672 d^{-1}$

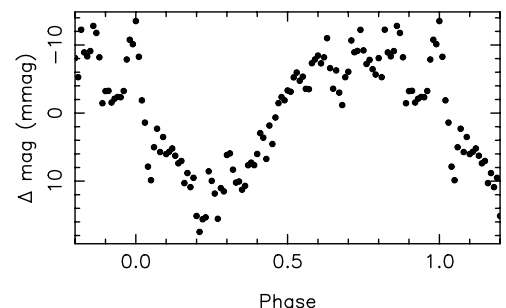

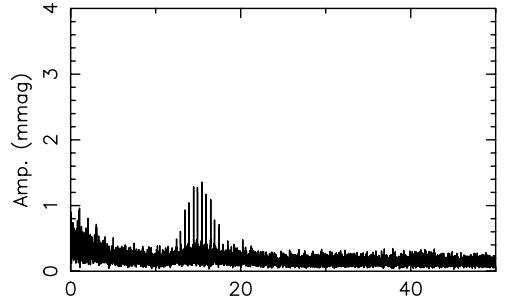

Freq. $\left(d^{-1}\right)$

Frequency $15.4668531 d^{-1}$

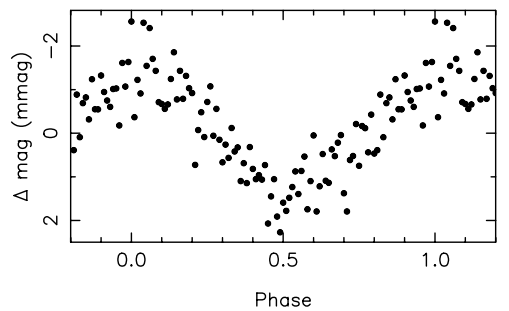

Ren ID 41640 (1SWASPJ162309.65-340110.9)

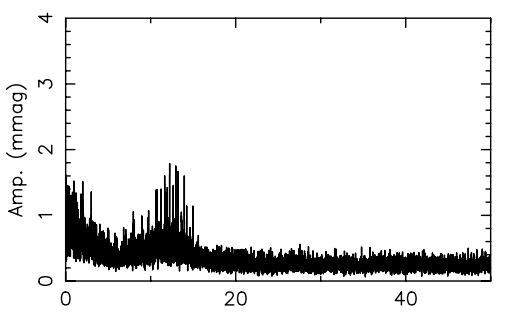

Freq. $\left(d^{-1}\right)$

Frequency $12.2295198 d^{-1}$

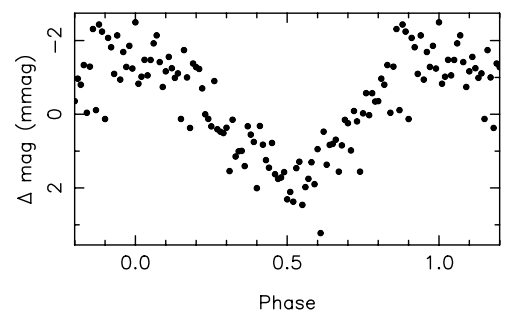

Ren ID 45870 (1SWASPJ174919.66+464100.0)

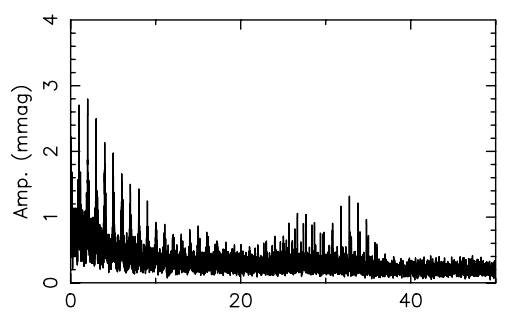

Freq. $\left(d^{-1}\right)$

Frequency $32.7894058 d^{-1}$

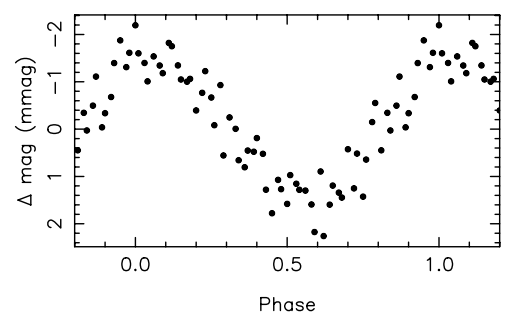

Fig. 1. continued. 
B. Smalley et al.: SuperWASP observations of pulsating Am stars

Ren ID 46050 (1SWASPJ175035.09+445841.3)

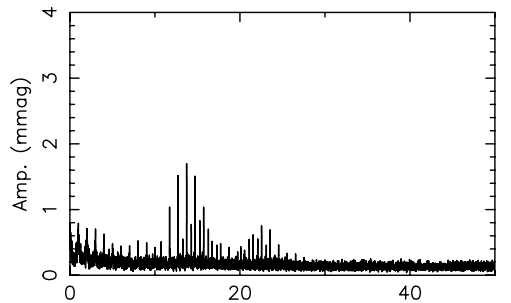

Freq. $\left(d^{-1}\right)$

Frequency $13.7280264 \mathrm{~d}^{-1}$

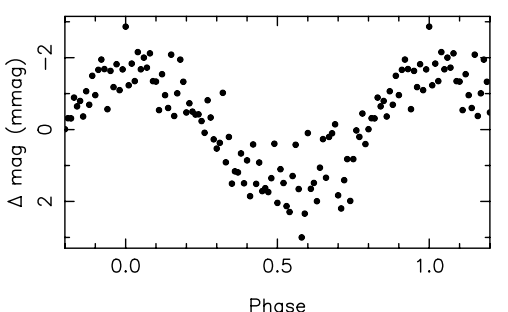

Ren ID 50420 (1SWASPJ192136.03+450706.8)

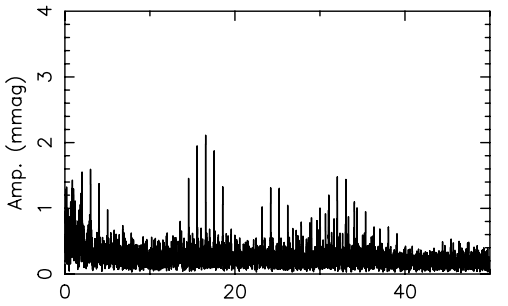

Freq. $\left(d^{-1}\right)$

Frequency $16.5565147 d^{-1}$

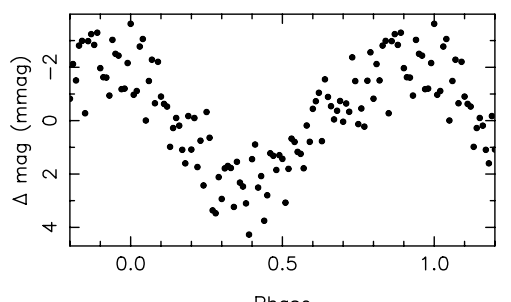

Ren ID 51760 (1SWASPJ195242.34-344101.0)

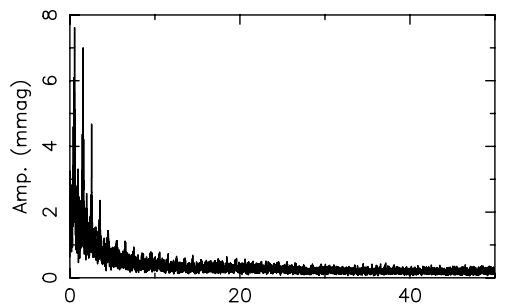

Freq. $\left(d^{-1}\right)$

Frequency $0.546822071 \mathrm{~d}^{-1}$

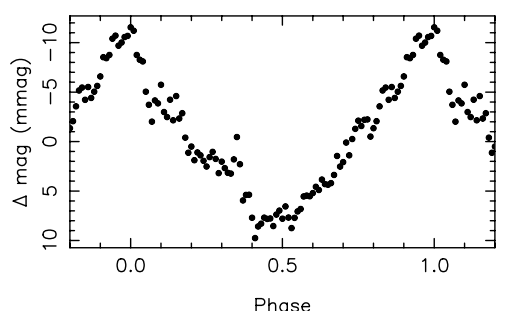

Ren ID 49650 (1SWASPJ190540.61+491820.7)

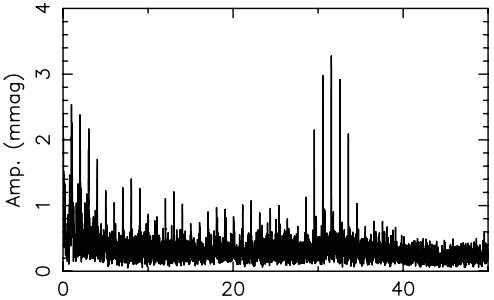

Freq. $\left(d^{-1}\right)$

Frequency $31.5577011 \mathrm{~d}^{-1}$

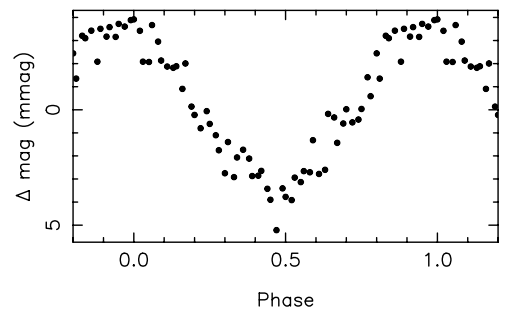

Ren ID 50670 (1SWASPJ192732.81+491523.5)

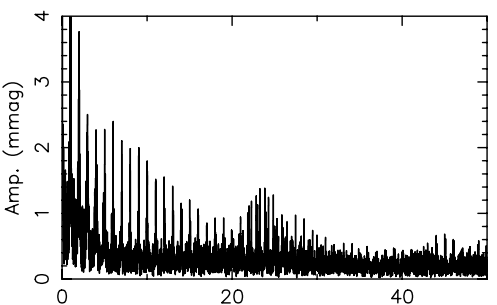

Freq. $\left(d^{-1}\right)$

Frequency $23.8463612 d^{-1}$

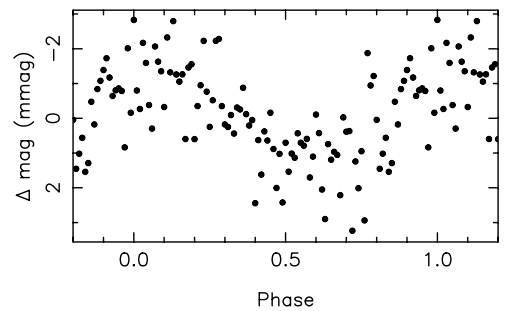

Ren ID 52850 (1SWASPJ200537.89-395410.2)

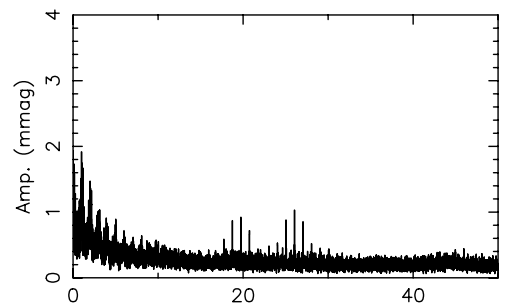

Freq. $\left(d^{-1}\right)$

Frequency $26.0472679 d^{-1}$

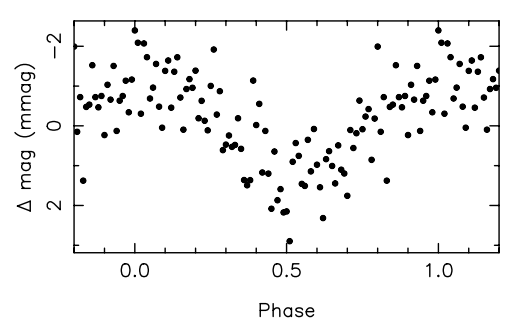

Ren ID 50230 (1SWASPJ191724.91+463535.2)

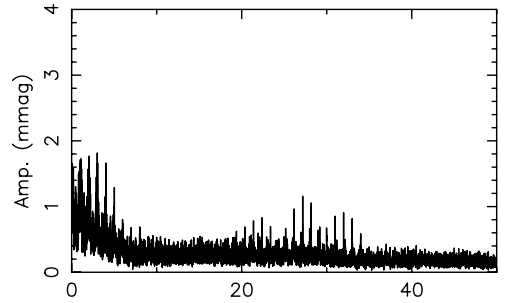

Freq. $\left(d^{-1}\right)$

Frequency $27.1776295 d^{-1}$

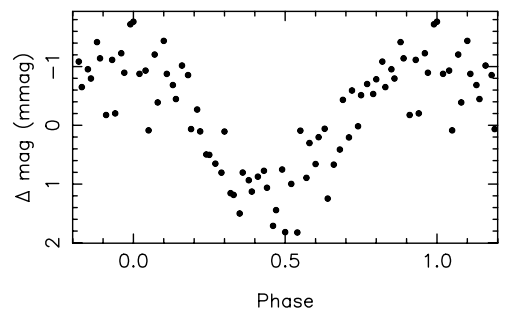

Ren ID 50520 (1SWASPJ192758.46-452231.6)

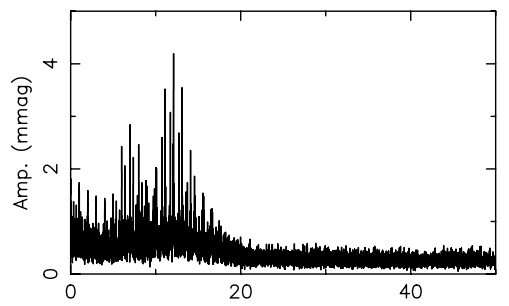

Freq. $\left(d^{-1}\right)$

Frequency $12.1003466 d^{-1}$

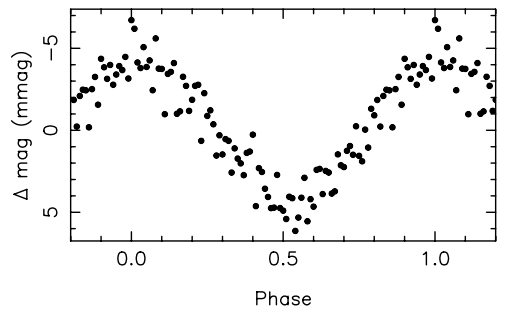

Ren ID 54150 (1SWASPJ202432.93-362548.6)

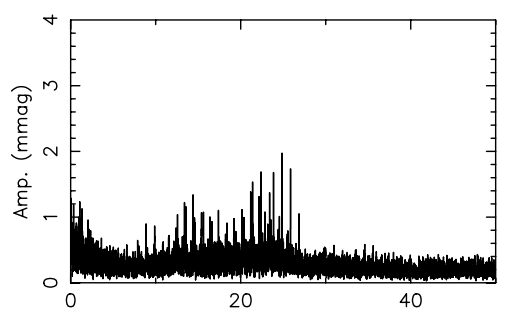

Freq. $\left(d^{-1}\right)$

Frequency $24.8629322 d^{-1}$

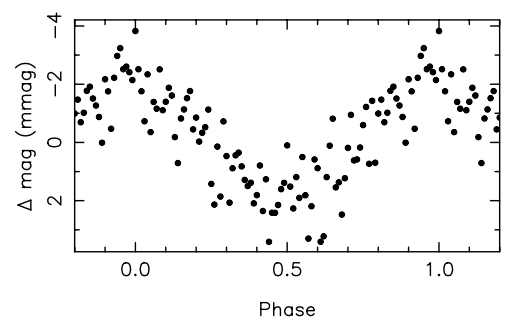

Fig. 1. continued. 


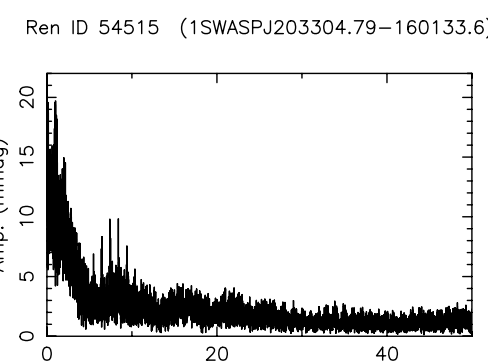

Freq. $\left(d^{-1}\right)$

Frequency $8.40408516 \mathrm{~d}^{-1}$

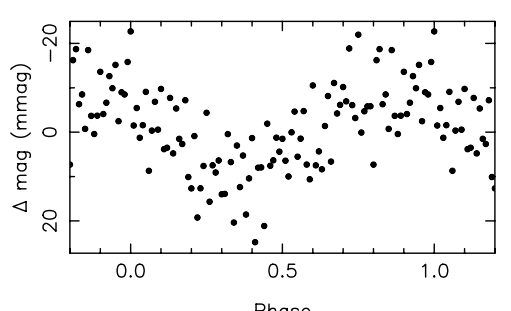

Ren ID 54970 (1SWASPJ204132.81-000037.8)

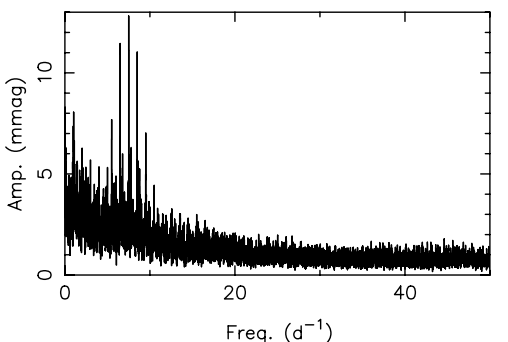

Frequency $7.50226974 \mathrm{~d}^{-1}$

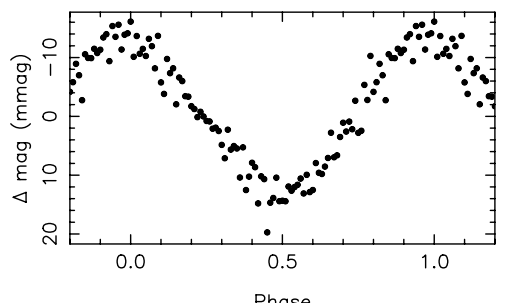

Ren ID 55710 (1SWASPJ210223.53-404934.8)

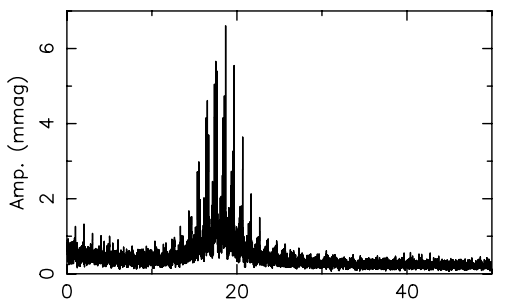

Freq. $\left(d^{-1}\right)$

Frequency $18.6733665 d^{-1}$

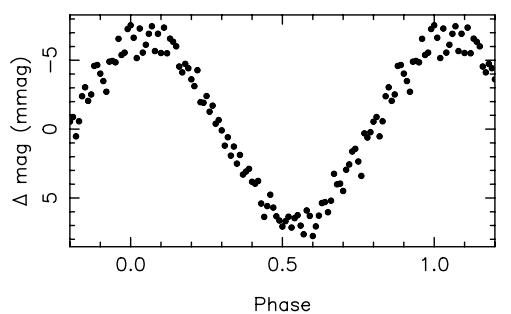

Ren ID 54656 (1SWASPJ203539.69-171341.4)

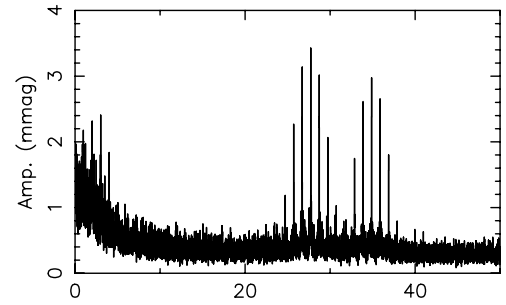

Freq. $\left(d^{-1}\right)$

Frequency $27.7230682 d^{-1}$

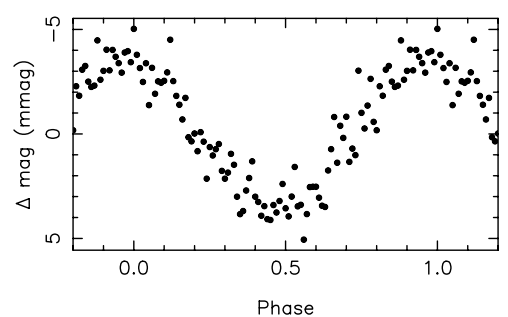

Ren ID 55087 (1SWASPJ204316.11+515100.9)

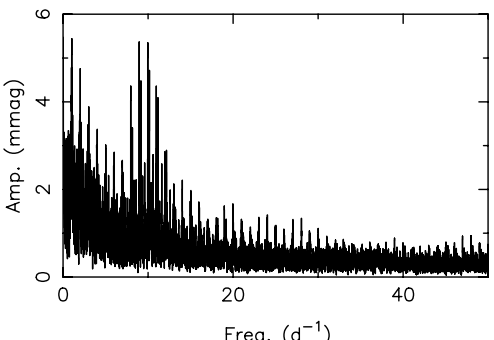

Frequency $8.95889568 d^{-1}$

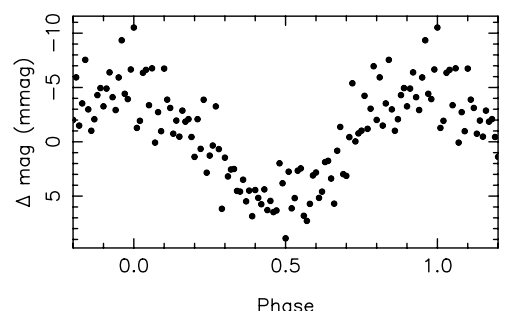

Ren ID 56159 (1SWASPJ210759.94+374105.0)

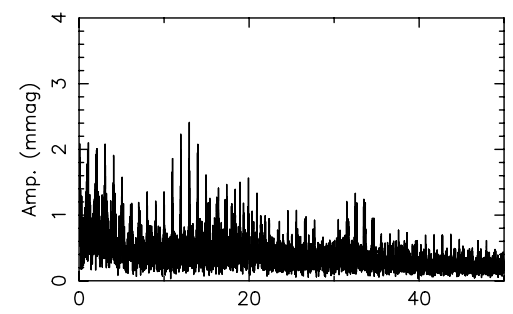

Freq. $\left(d^{-1}\right)$

Frequency $12.9635162 d^{-1}$

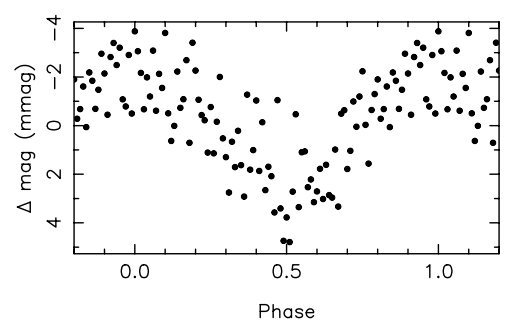

Ren ID 54736 (1SWASPJ203748.56-184633.8)

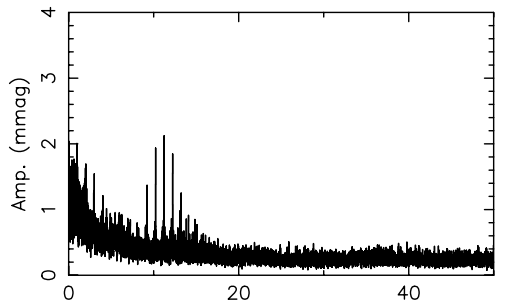

Freq. $\left(d^{-1}\right)$

Frequency $11.228754 \mathrm{~d}^{-1}$

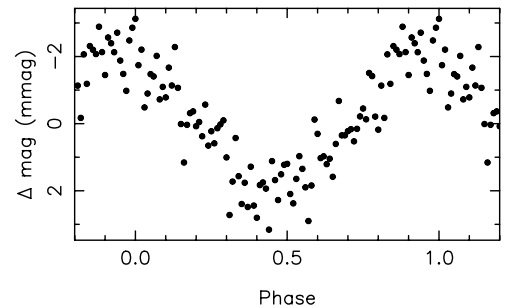

Ren ID 55094 (1SWASPJ204610.15-113617.0)

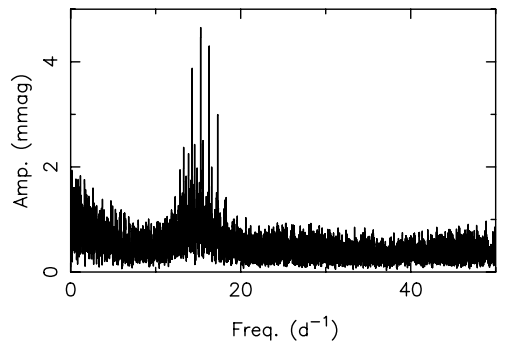

Frequency $15.2904186 d^{-1}$

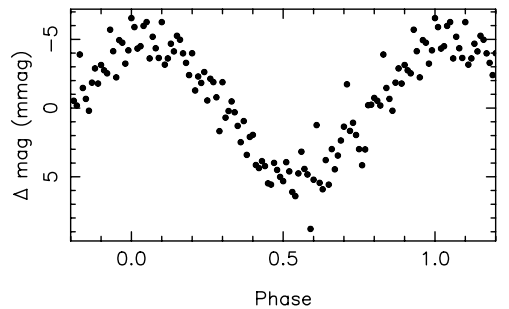

Ren ID 56110 (1SWASPJ210904.35-404648.2)

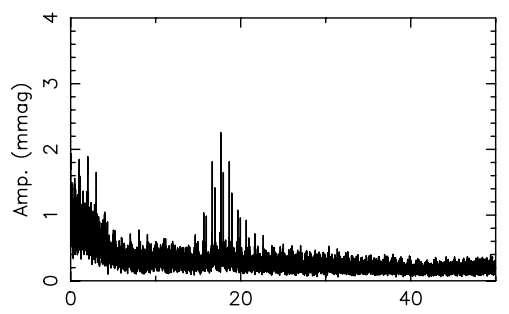

Freq. $\left(d^{-1}\right)$

Frequency $17.6570873 d^{-1}$

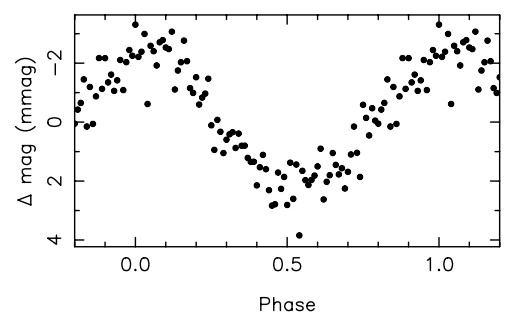

Fig. 1. continued. 
B. Smalley et al.: SuperWASP observations of pulsating Am stars

Ren ID 56275 (1SWASPJ211108.55+344906.2)

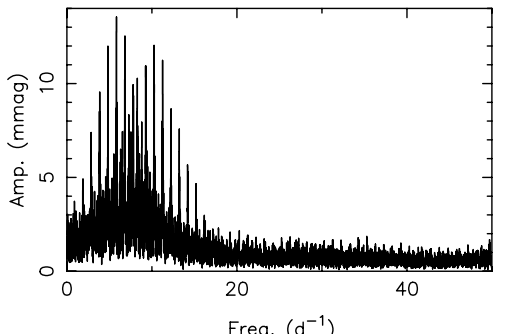

Frequency $5.82577038 \mathrm{~d}^{-1}$

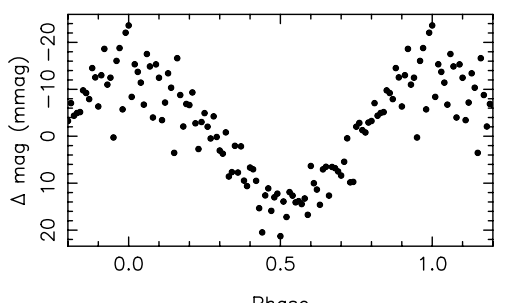

Ren ID 56980 (1SWASPJ213050.87-383048.8)

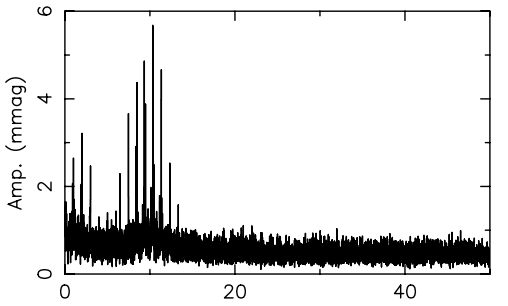

Freq. $\left(d^{-1}\right)$

Frequency $10.3384886 d^{-1}$

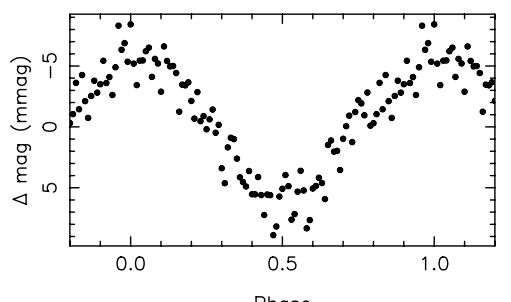

Ren ID 57300 (1SWASPJ213748.56-374203.5)

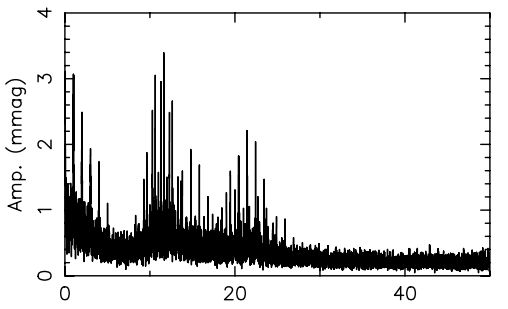

Freq. $\left(d^{-1}\right)$

Frequency $11.6332283 d^{-1}$

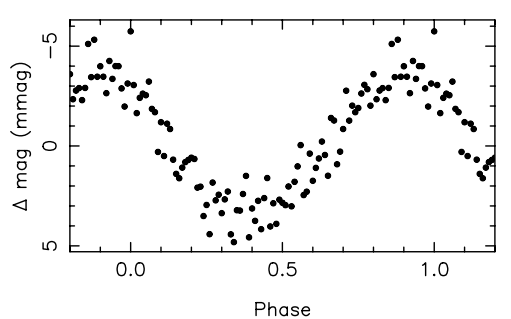

Ren ID 56280 (1SWASPJ211611.35-701356.3)

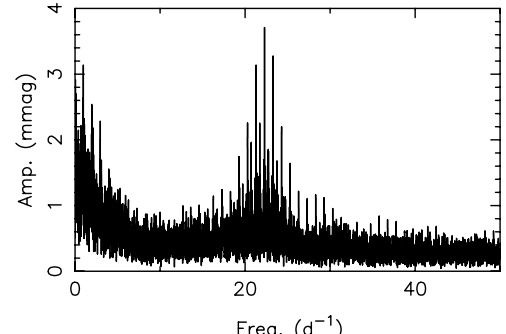

Frequency $22.2878685 \mathrm{~d}^{-1}$

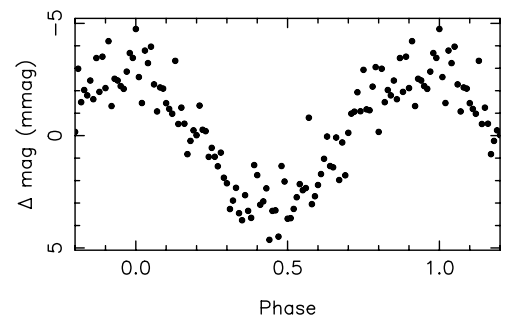

Ren ID 57020 (1SWASPJ213202.68-374611.4)

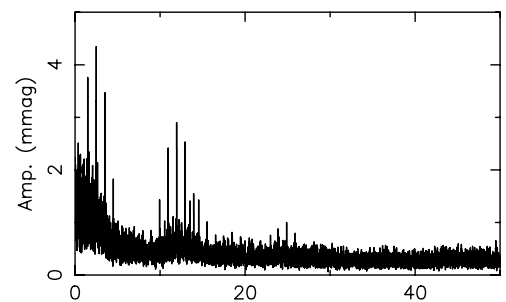

Freq. $\left(d^{-1}\right)$

Frequency $2.49128604 d^{-1}$

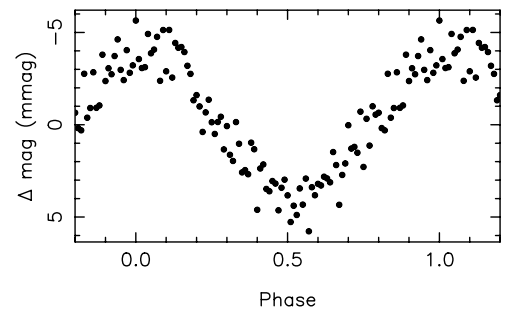

Ren ID 57323 (1SWASPJ213807.80-102620.6)

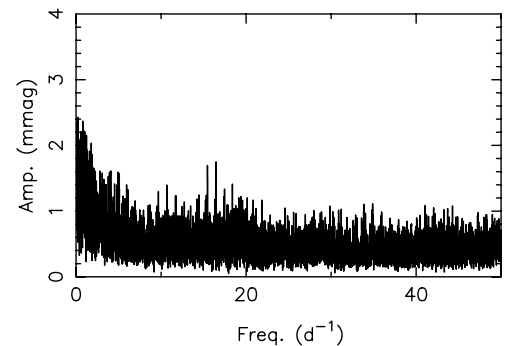

Frequency $16.459816 d^{-1}$

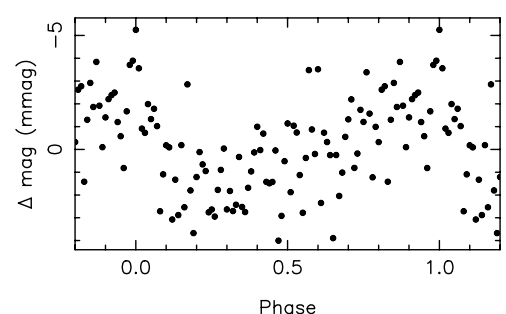

Ren ID 56770 (1SWASPJ212624.52-465908.4）

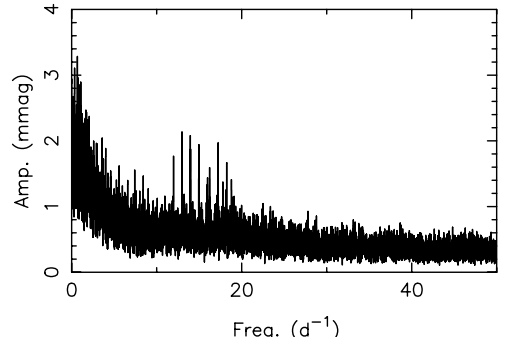

Frequency $12.9822912 \mathrm{~d}^{-1}$

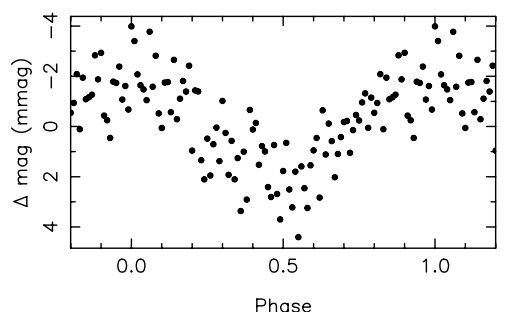

Ren ID 57104 (1SWASPJ213239.02-214635.3)

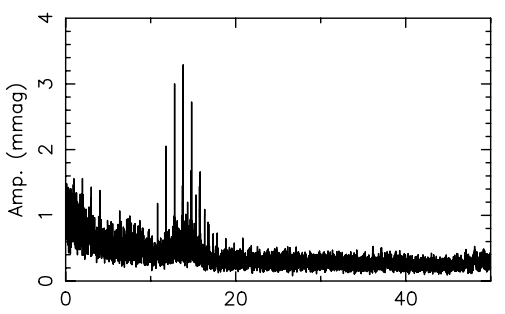

Freq. $\left(d^{-1}\right)$

Frequency $13.8157835 d^{-1}$

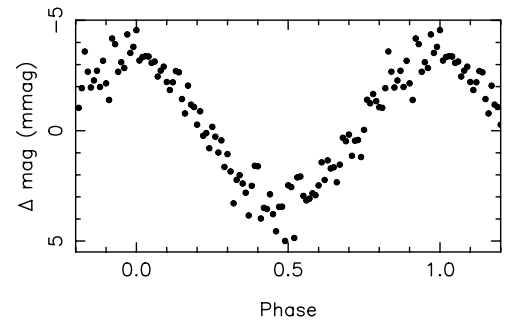

Ren ID 57696 (1SWASPJ214551.51+572821.0)

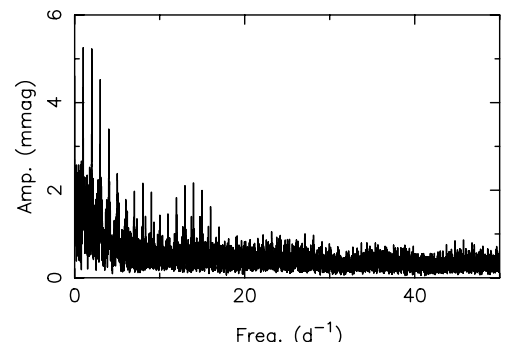

Frequency $13.9770193 d^{-1}$

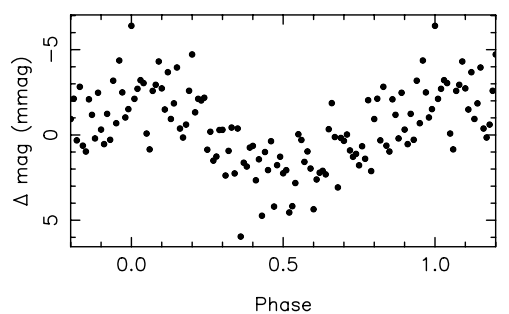

Fig. 1. continued. 


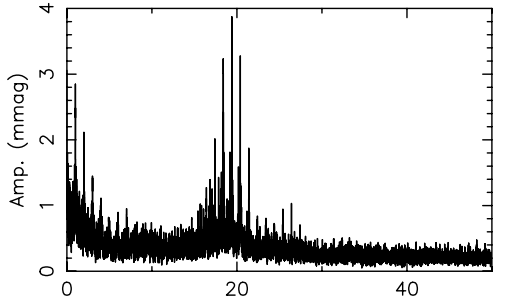

Freq. $\left(d^{-1}\right)$

Frequency $19.3943501 d^{-1}$

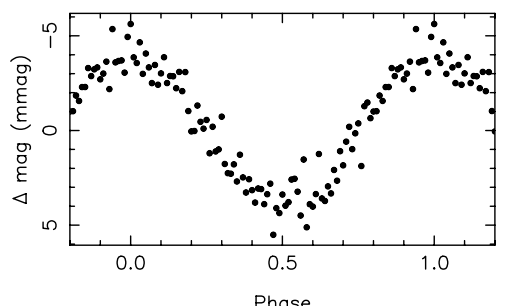

Ren ID 58440 (1SWASPJ220927.90-675503.7)

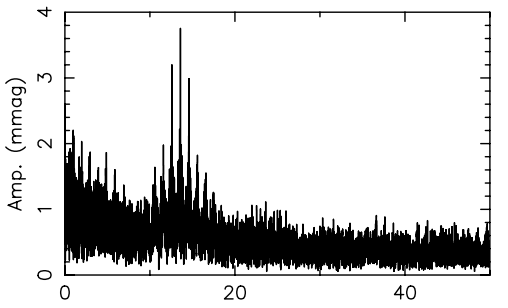

Freq. $\left(d^{-1}\right)$

Frequency $13.589242 \mathrm{~d}^{-1}$

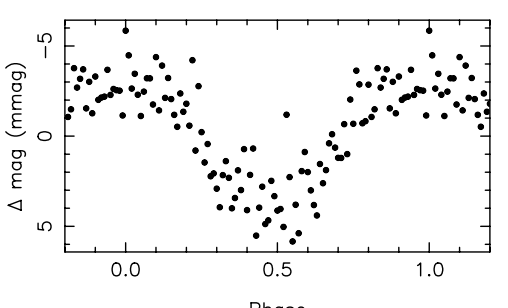

Ren ID 59072 (1SWASPJ222703.19+481048.9)

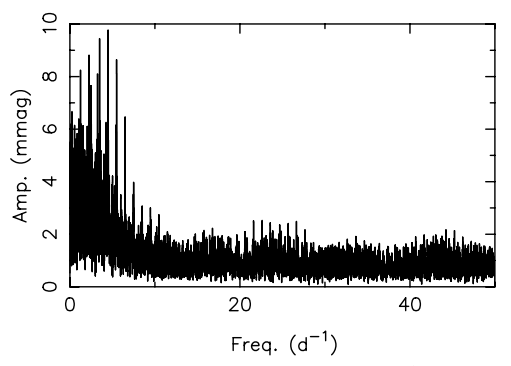

Frequency $4.48642159 \mathrm{~d}^{-1}$

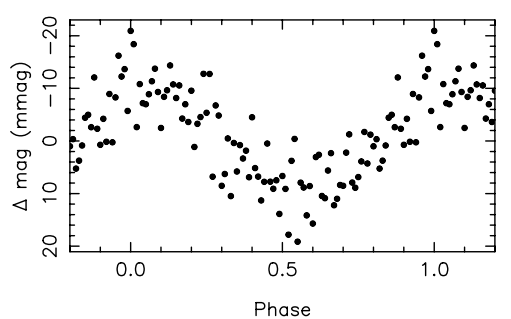

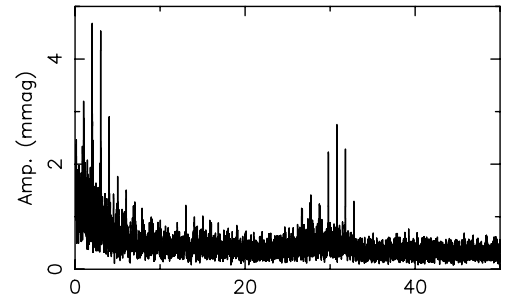

Freq. $\left(d^{-1}\right)$

Frequency $30.8010502 d^{-1}$

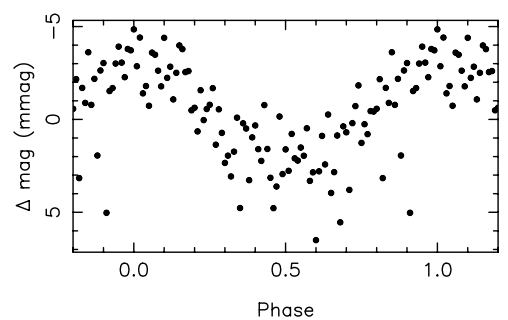

Ren ID 58850 (1SWASPJ222133.12+240418.9)

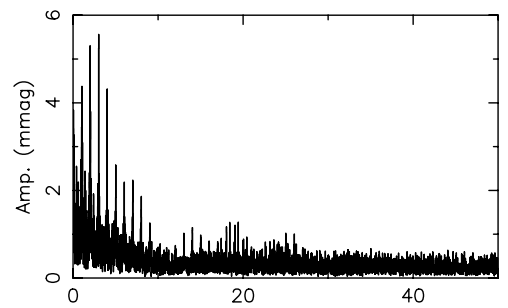

Freq. $\left(d^{-1}\right)$

Frequency $19.4303436 d^{-1}$

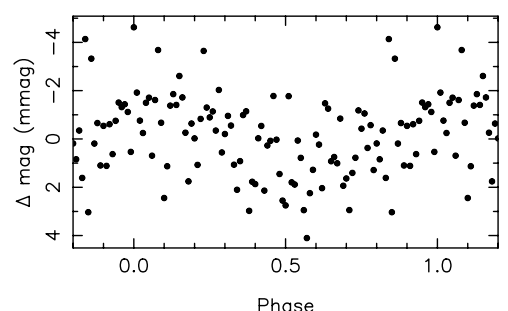

Ren ID 59020 (1SWASPJ222716.30-343617.8)

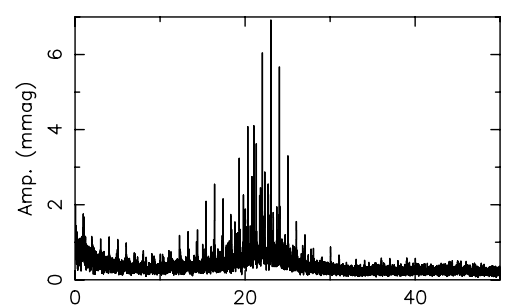

Freq. $\left(d^{-1}\right)$

Frequency $23.0318241 \mathrm{~d}^{-1}$

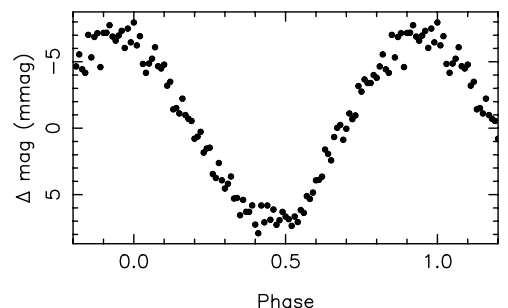

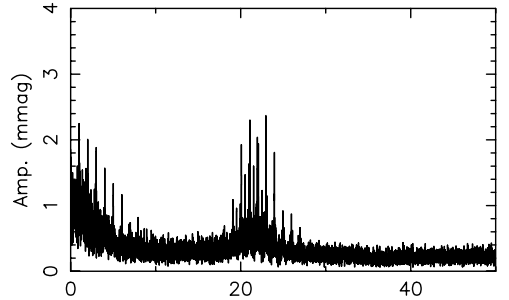

Freq. $\left(d^{-1}\right)$

Frequency $22.965292 \mathrm{~d}^{-1}$

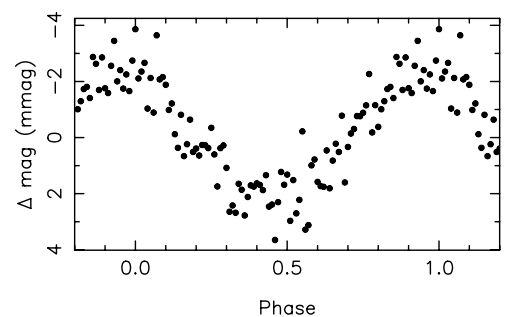

Ren ID 58870 (1SWASPJ222246.83-335606.6)

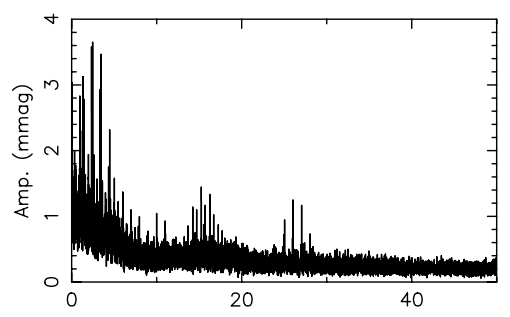

Freq. $\left(d^{-1}\right)$

Frequency $2.46216679 d^{-1}$

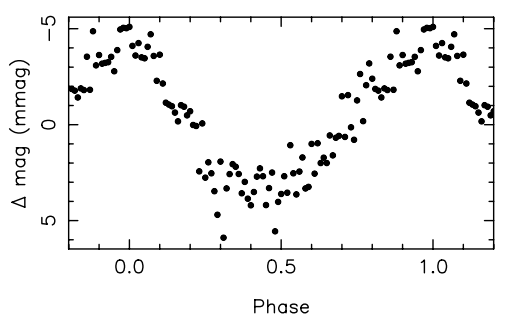

Ren ID 59090 (1SWASPJ223012.81-302620.2)

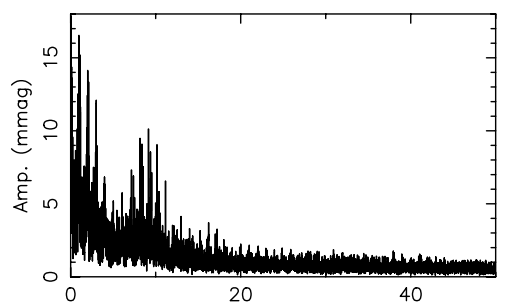

Freq. $\left(d^{-1}\right)$

Frequency $9.15213966 d^{-1}$

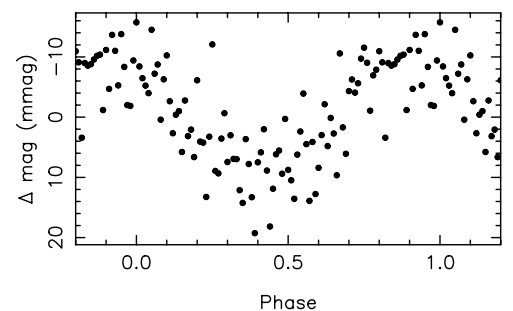

Fig. 1. continued. 
B. Smalley et al.: SuperWASP observations of pulsating Am stars

Ren ID 59500 (1SWASPJ224518.32-332133.9)

Ren ID 59560 (1SWASPJ224556.80+371209.6)

Ren ID 60696 (1SWASPJ233215.42-095301.8)

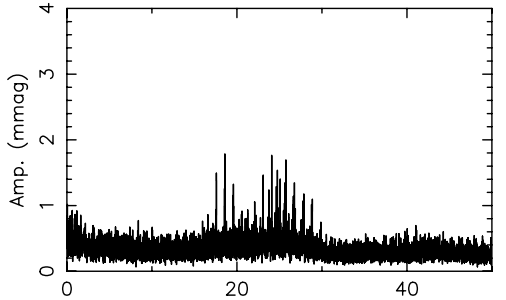

Freq. $\left(d^{-1}\right)$

Frequency $18.5844765 \mathrm{~d}^{-1}$

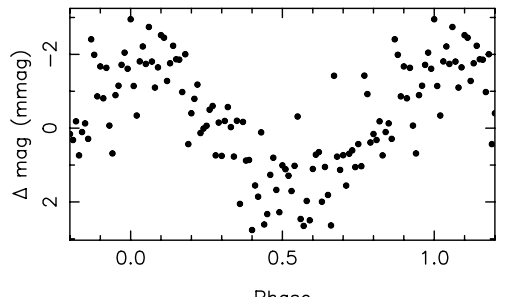

Ren ID 60690 (1SWASPJ233218.49-334033.1)

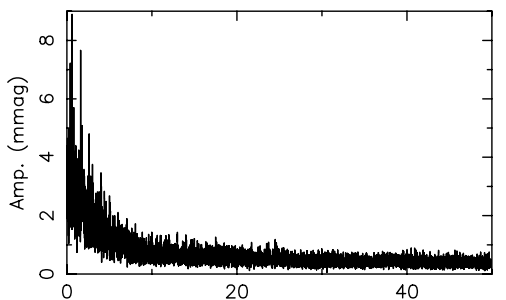

Freq. $\left(d^{-1}\right)$

Frequency $0.607181609 d^{-1}$

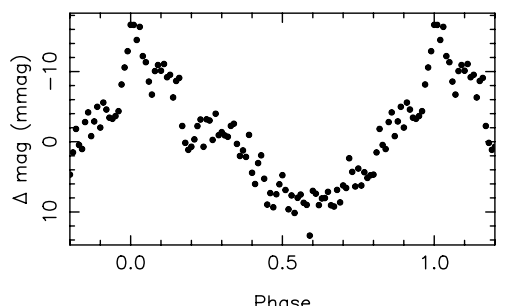

Ren ID 61320 (1SWASPJ235143.20-370546.5)

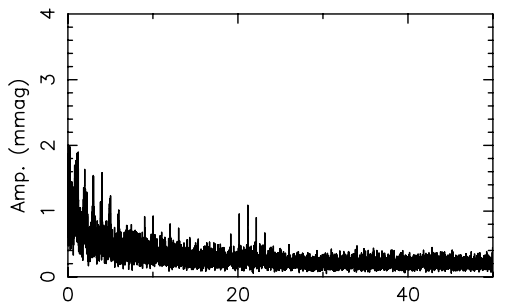

Freq. $\left(d^{-1}\right)$

Frequency $21.1611595 d^{-1}$

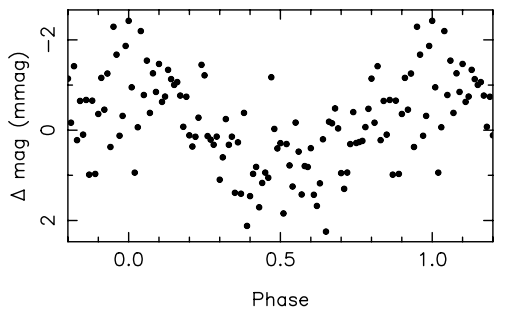

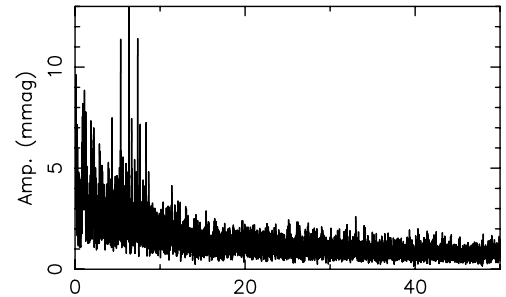

Freq. $\left(d^{-1}\right)$

Frequency $6.35844707 d^{-1}$

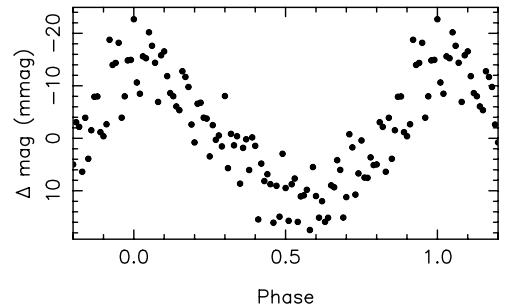

Ren ID 60740 (1SWASPJ233319.47-140859.9)

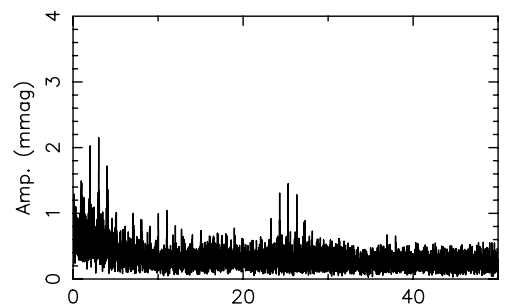

Freq. $\left(d^{-1}\right)$

Frequency $25.3062878 d^{-1}$

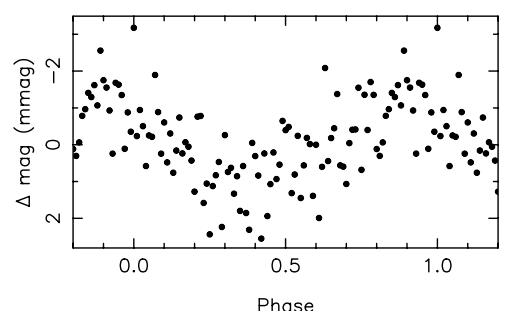

Ren ID 61350 (1SWASPJ235310.92+450225.9)

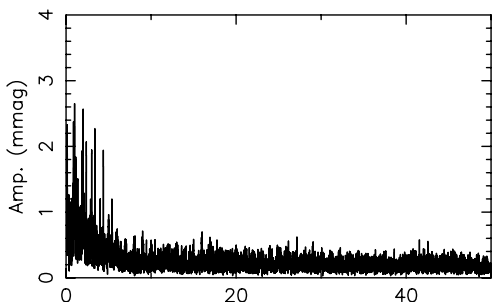

Freq. $\left(d^{-1}\right)$

Frequency $3.38020802 \mathrm{~d}^{-1}$

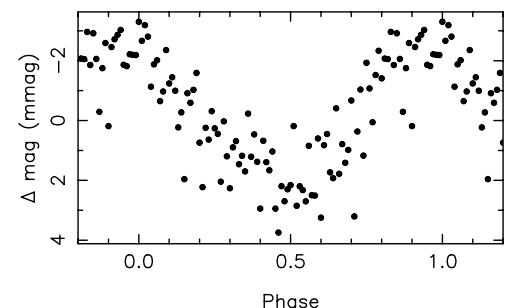

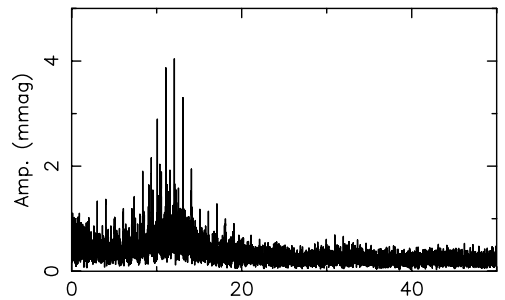

Freq. $\left(d^{-1}\right)$

Frequency $12.0775261 d^{-1}$

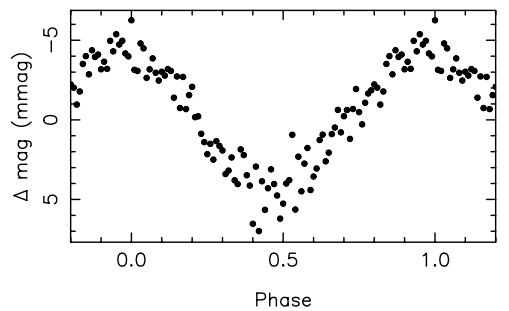

Ren ID 61105 (1SWASPJ234358.16-244553.1)

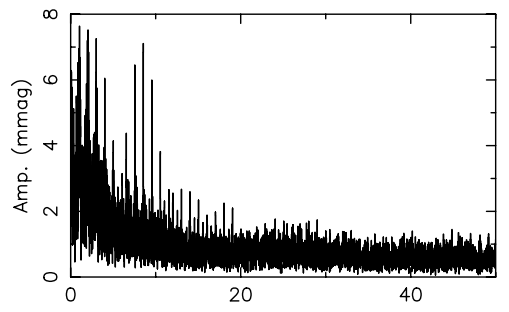

Freq. $\left(d^{-1}\right)$

Frequency $8.54540348 d^{-1}$

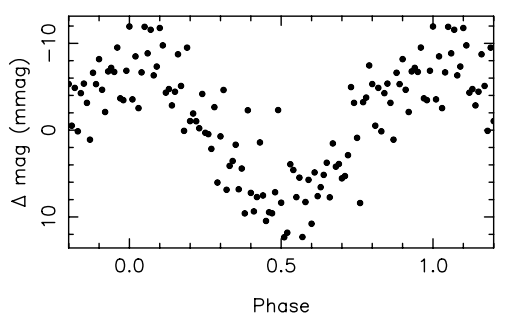

Ren ID 61356 (1SWASPJ235349.63-011701.3)

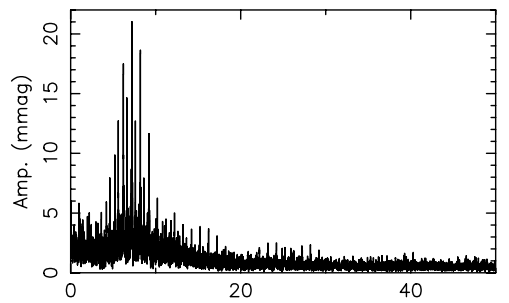

Freq. $\left(d^{-1}\right)$

Frequency $7.19764662 d^{-1}$

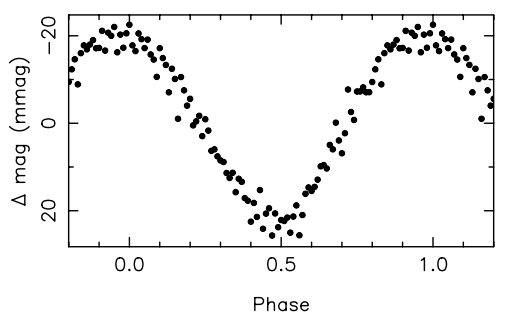

Fig. 1. continued. 
A\&A 535, A3 (2011)

Ren ID 61580 (1SWASPJ235943.25+211720.5)

Ren ID 61756 (1SWASPJ000411.09-045533.9)

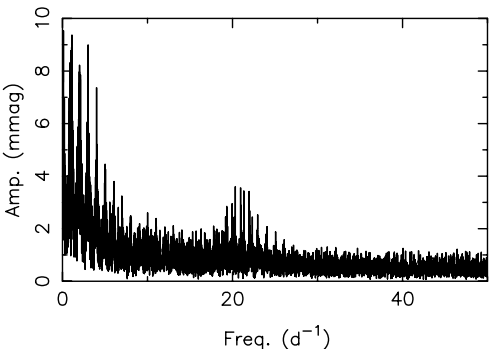

Frequency $20.3278294 d^{-1}$

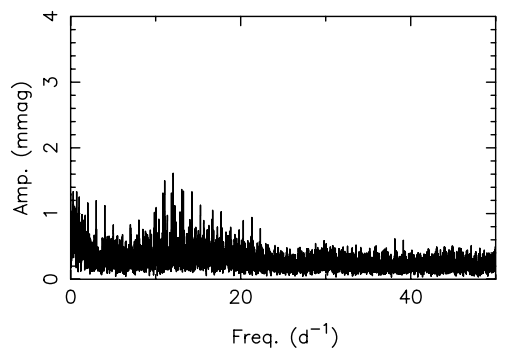

Frequency $12.0587893 d^{-1}$
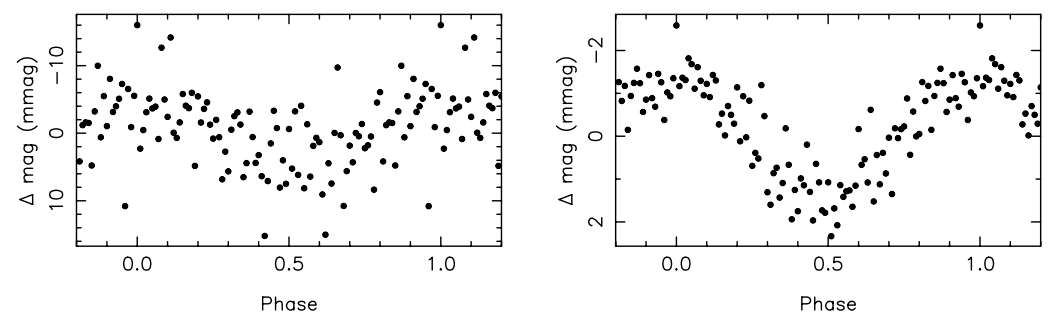

Fig. 1. continued. 